\title{
Stigma by association : among family members of people with mental illness
}

Citation for published version (APA):

van der Sanden, R. L. M. (2015). Stigma by association : among family members of people with mental illness. [Doctoral Thesis, Maastricht University]. Datawyse / Universitaire Pers Maastricht. https://doi.org/10.26481/dis.20150611rs

Document status and date:

Published: 01/01/2015

DOI:

10.26481/dis.20150611rs

Document Version:

Publisher's PDF, also known as Version of record

\section{Please check the document version of this publication:}

- A submitted manuscript is the version of the article upon submission and before peer-review. There can be important differences between the submitted version and the official published version of record.

People interested in the research are advised to contact the author for the final version of the publication, or visit the DOI to the publisher's website.

- The final author version and the galley proof are versions of the publication after peer review.

- The final published version features the final layout of the paper including the volume, issue and page numbers.

Link to publication

\footnotetext{
General rights rights.

- You may freely distribute the URL identifying the publication in the public portal. please follow below link for the End User Agreement:

www.umlib.nl/taverne-license

Take down policy

If you believe that this document breaches copyright please contact us at:

repository@maastrichtuniversity.nl

providing details and we will investigate your claim.
}

Copyright and moral rights for the publications made accessible in the public portal are retained by the authors and/or other copyright owners and it is a condition of accessing publications that users recognise and abide by the legal requirements associated with these

- Users may download and print one copy of any publication from the public portal for the purpose of private study or research.

- You may not further distribute the material or use it for any profit-making activity or commercial gain

If the publication is distributed under the terms of Article $25 \mathrm{fa}$ of the Dutch Copyright Act, indicated by the "Taverne" license above, 


\section{STIGMA BY ASSOCIATION}

Among family members of

people with mental illness

Remko van der Sanden
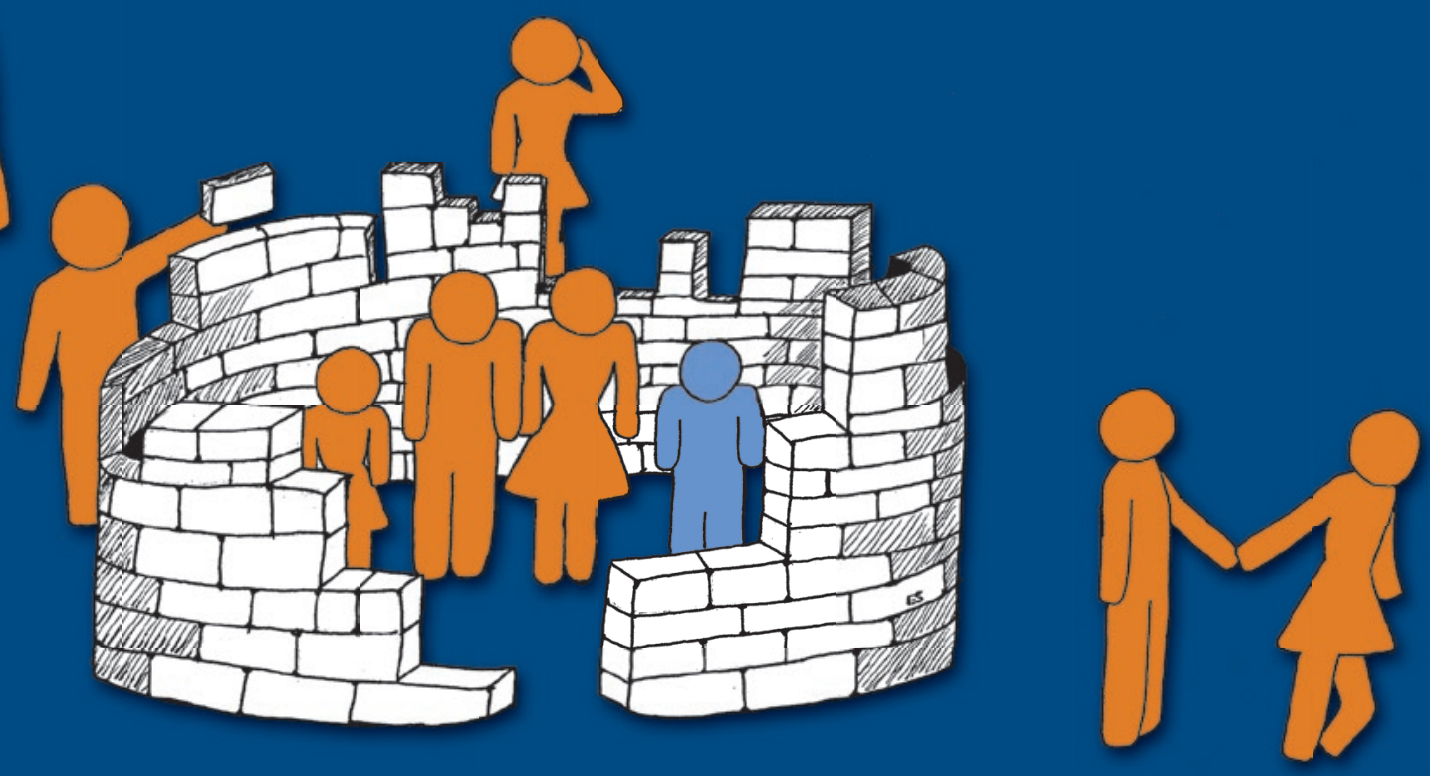
Stigma by association among family members of people with mental illness

R.L.M. van der Sanden

All rights reserved. No part of this publication may be reproduced, stored in a retrieval system, transmitted in any form, or by any means, electronic or mechanical, including photocopying, recording or by any other information storage or retrieval system, without the prior written permission of the author.

ISBN 9789461594341

Copyright (C) R.L.M. van der Sanden, Maastricht 2015

Cover design: Ellen van der Sanden

Printed by: Datawyse | Universitaire Pers Maastricht 


\title{
STIGMA BY ASSOCIATION
}

\author{
AMONG FAMILY MEMBERS OF \\ PEOPLE WITH MENTAL ILLNESS
}

\author{
PROEFSCHRIFT \\ ter verkrijging van de graad van doctor aan de Universiteit Maastricht, \\ op gezag van de Rector Magnificus, Prof. Dr. L.L.G. Soete \\ volgens het besluit van het College van Decanen, \\ in het openbaar te verdedigen \\ op donderdag 11 juni 2015 om 12.00 uur \\ door \\ Remko Leonard Maria van der Sanden
}

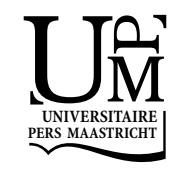




\section{Promotores}

Prof. dr. G. Kok

Prof. dr. J. B. Pryor (Illinois State University, USA)

\section{Copromotores}

Dr. A. E. R. Bos (Open Universiteit Nederland)

Dr. S. E. Stutterheim (Open Universiteit Nederland)

\section{Beoordelingscommissie}

Prof. dr. R. A. C. Ruiter, voorzitter

Prof. dr. P. W. Corrigan (Illinois Institute of Technology, USA)

Dr. J. S. M. Krumeich

Prof. dr. J. J. D. M. van Lankveld (Open Universiteit Nederland)

Prof. dr. F. J. N. Nijhuis 
to Janny 



\section{TABLE OF CONTENTS}

CHAPTER 1: Introduction 9

CHAPTER 2: Experiences of stigma by association among family members of people with mental illness

CHAPTER 3: Stigma by association among family members of people with a mental illness: A qualitative analysis

CHAPTER 4: Coping with stigma by association and family burden among family members of people with mental illness

CHAPTER 5: Harmful effects of stigma by association and family burden among family members of people with mental illness: The mediating role of coping

CHAPTER 6: General discussion

REFERENCES

SUMMARY

SAMENVATTING

147

VALORISATION 153

ACKNOWLEDGEMENTS

163

CURRICULUM VITAE 

CHAPTER 1

INTRODUCTION 
"Avigdor, I just came from her, what happened? The wedding is off. Why? He says I have the evil eye, he says a streak of melancholy runs in our blood. What are you saying? No man can let his daughter marry into a family like that, it's forbidden. I have no idea what you are talking about. They found out. What? About my brother! What about your brother? He did not die of pneumonia or consumption, I lied, he committed suicide. So, no wedding, no sons, what's all this for?"

From: Yentl (Streisand, DeWaay, Lemorande, \& Streisand, 1983) 


\section{INTRODUCTION}

According to estimates by the World Health Organization [WHO] over 450 million people worldwide suffer from mental illness, and one in four families are likely to have at least one family member with mental illness (WHO, 2001; WHO, 2014). Presently, in most publications and factsheets, numbers still range from 100 million to 500 million or higher. Furthermore, it is estimated that, in any given year, about $26.2 \%$ of adult Americans suffer from mental illness (Kessler, Chiu, Demler, \& Walters, 2005), and the Netherlands Mental Health Survey and Incidence Study conducted in 2010 demonstrated that about $42 \%$ of Dutch adults between 18 and 65 have suffered from an axis1 mental illness at some point in their lives (De Graaf, Ten Have, \& Van Dorsselaer, 2010). The number of people worldwide with mental illness as such is considerable, and they and their family members face many problems, barriers, and disadvantages (Butter, Webster, \& Hill, 2010). For example, people with mental illness are more likely to be unemployed, have less income, experience more psychological distress, receive less social support, and have a diminished quality of life (Bos, Kanner, Muris, Janssen, \& Mayer, 2009; Link \& Cullen, 1990).

Many mental illnesses first occur during adolescence when one still lives in the parental home together with immediate family members (Kessler, Berglund, Demler, Jin, Merikangas, \& Walters, 2005). As such, the mental health condition is likely to affect not only the person in question but also the lives of immediate family members. Even when a person with mental illness is older, has moved out and has an independent household, his or her mental health condition is still likely to affect the lives of family members. In fact, mental illnesses are known to have 'a ripple and domino effect'. They can create tensions, uncertainties, changes, burden, negative emotions, and troubles within families (Farina, Fischer, \& Fischer, 1992; Judge, 1994). The daily lives of family members of people with mental illness are further altered by the fact that they often provide day-to-day care, performing necessary caregiver activities (Sales, 2003).

Among the many hardships endured by people who have a family member with mental illness is the stigma they share, and mental illnesses are among the most severely stigmatised health conditions in our society. People with mental illness and their family members are frequently stigmatised by the general public (Bos et al., 2013; Corrigan \& Penn, 1999; Corrigan \& Miller, 2004; Fink \& Tasman, 1992), and although experiences of stigmatisation are pervasive among people with mental illness, the impact of these experiences varies widely both among individuals and among family members (Corrigan \& Miller, 2004; Corrigan, Watson, \& Miller, 2006). In this dissertation, the stigmatisation as experienced by family members of people with mental illness is examined, as 
are the consequences of these experiences and the ways in which family members cope with experiences of stigmatisation.

\section{Stigma}

The term stigma itself refers to a discrediting or disgraceful mark that sets individuals apart from others and renders them tainted, degraded, or inferior in the eyes of other people (Bos, Kok, \& Dijker, 2001; Crocker, Major, \& Steele, 1998). It originally dates back to ancient Greece where it was a mark made by a branding iron or a tattoo on the skin of a slave, criminal, or traitor to identify that person as a degraded or immoral person that should be avoided (Bos et al., 2001; Crocker et al., 1998). Today, stigmatisation is a complex social process. It refers to oversimplified conceptions, opinions, or images about a person or group (stereotypes), negative attitudes that reflect such stereotypes (prejudice), and overt negative behaviour (discrimination) towards people with a stigmatised condition (Brohan, Slade, Clement, \& Thornicroft, 2010; Corrigan, 2005; Phelan, Link, \& Dovidio, 2008). Many definitions of stigma consist of two components: difference and devaluation. To trigger stigmatising reactions from others, the stigma should be noticeable (difference) and decrease one's social status (devaluation; Bos et al., 2013; Dovidio, Major, \& Crocker, 2000). Additionally, Phelan et al. (2008) claims that stigmatisation also serves several social purposes, namely "exploitation and domination (keeping people down); norm enforcement (keeping people in); and disease avoidance (keeping people away)" (p. 358).

Evidently, stigma is not merely a physical mark, but is often seen as a context related attribute that leads to widespread social disapproval (Bos et al., 2013; Byrne, 2000; Stutterheim, 2011). As such, stigma does not reside in a person but occurs within a social context and, more specifically, in social interactions between people. Consequently, experiences constituted as stigmatising in one social context may not be perceived as stigmatising in another (Bos et al., 2013; Crocker et al., 1998, Dovidio et al., 2000). Manifestations of stigma may be overt and include repulsion, disgust, avoidance, rejection, and the dehumanisation, degradation, discredit, and depersonalisation of others (Bos, 2001; Dovidio et al., 2000). Stigmatisation may also manifest more subtly. For instance, people may display their underlying and implicit emotions and feelings through nonverbal expressions of distress, anxiety, or unease (Bos, 2001). Whether explicit or subtle, stigmatisation can lead to negative consequences such as social exclusion, poor treatment, and other negative social interactions with members of the general public.

Pryor and Reeder (2011) developed a conceptual model that describes four dynamic, interrelated manifestations of stigma (see Figure 1.1). 


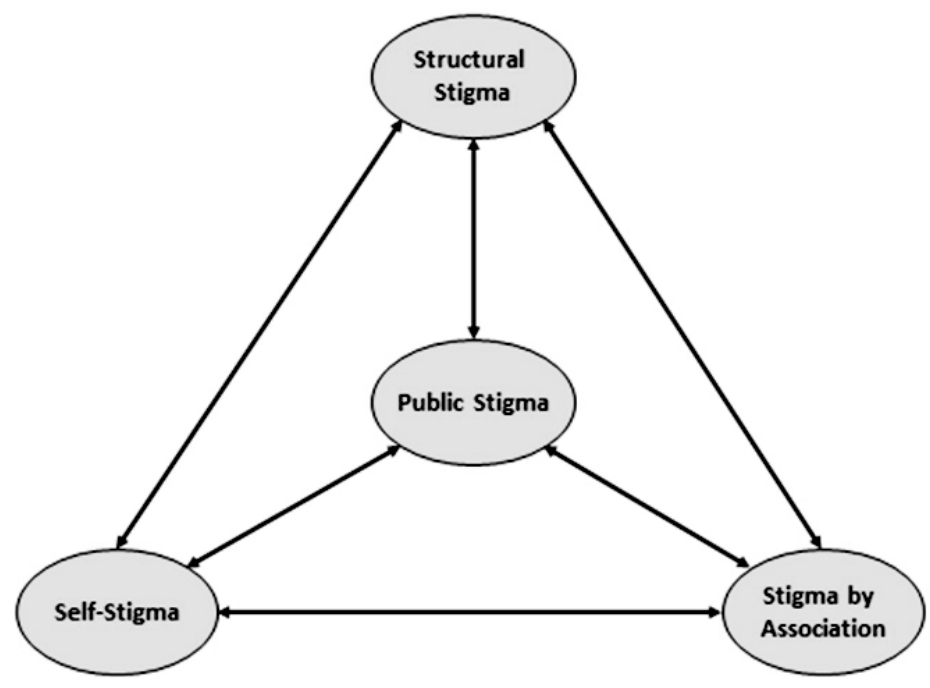

Figure 1.1: Four types of stigma (based on Pryor \& Reeder, 2011).

Public stigma is at the core of this model and comprises emotional and behavioural reactions to people with a stigmatised condition, as well as the cognitive representations held by the general public towards people assumed to have a stigmatised condition (Stutterheim, 2011). Public stigma has been shown to elicit negative emotional and behavioural reactions from others that result in stigmatising behaviour such as avoidance, blame, and rejection, that, in turn, can manifest as discrimination, exclusion, or other negative social interactions (Black \& Miles, 2002; Corrigan, Larson, \& Kuwabara, 2007; Corrigan \& Watson, 2002). Hence, public stigma contains cognitive, affective, and behavioural elements that reflect the perspective of those who stigmatise (Stutterheim, 2011). The negative opinions and reactions on the part of the general public are called public stigma (Black \& Miles, 2002; Corrigan et al., 2007; Michaels, López, Rüsch, \& Corrigan, 2012; Pescolido et al., 2013; Reeder \& Pryor, 2008).

Public stigma is an important type of stigma but not the only type of stigma. In fact, public stigma may "spill over" from persons with a stigmatised condition to people associated with them, such as family, friends, companions, and buddies. This is called stigma by association and is similar to what Goffman (1963) termed courtesy stigma and what Lefley (1989) called family stigma. In Pryor and Reeder's (2011) model, stigma by association represents the process through which previously non-stigmatised people are stigmatised and discredited by the general public because of their affiliation 
with a stigmatised person (Neuberg, Smith, Hoffman, \& Russell, 1994; Pryor, Reeder, \& Monroe, 2012).

The third type of stigma in Pryor and Reeder's (2011) model is self-stigma. Self-stigma includes both the fear of being exposed to stigmatisation, and the potential internalisation of stigmatising feelings and ideas, as well as the underlying assumptions associated with the stigmatised condition (Bos et al., 2013). Self-stigma occurs when stigmatised persons absorb the stigmatising beliefs and representations from the general public and come to believe them and internalise them. Thus, family members of people with mental illness who suffer from self-stigma may not only be exposed to stigmatising reactions and beliefs, they may also experience reduced self-esteem and selfefficacy as a result of self-stigma (Corrigan, Larson, \& Rüsch, 2009). In these cases, they are likely to suffer even more harm as a result of this self-stigma (Drapalski et al., 2013; West, Yanos, Smith, Roe, \& Lysaker, 2011).

The fourth component of Pryor and Reeder's (2011) model is called structural stigma. This includes the structural legitimatisation and perpetuation of public stigma or a general stigmatising situation via society's institutions, policies, legislation, and ideological systems (Bos et al., 2013; Pryor \& Reeder, 2011).

The four types of stigma proposed by Pryor and Reeder (2011) are interrelated, but public stigma is at the centre of the model and is considered to be the foundation for the three other manifestations: stigma by association, self-stigma, and structural stigma (Pryor \& Reeder, 2011).

\section{Public stigma and people with mental illness}

Pescolido et al. (2013) claimed that public stigma is currently still alive but perhaps somewhat more sophisticated than before; the general public seems to be more open to the recognition and disclosure of mental illnesses now than in previous years. Nonetheless, the stigmatisation of people with mental illness by the general public is still widespread in both Western societies and other cultures, and has detrimental effects on the mental health and quality of life of people with mental illness (Black \& Miles, 2002; Bos et al., 2009; Bos, Schaalma, \& Pryor, 2008; Corrigan et al., 2007; Crisp, Gelder, Rix, Meltzer, \& Rowlands, 2000; Dijker \& Koomen, 2003; Michaels et al., 2012; Pescolido et al., 2013).

When people are labelled as having a mental illness, they are often no longer seen as individuals but rather as members of a stereotyped group. The negative expectations, attitudes, and beliefs towards this group (e.g., a person with mental illness is less intelligent, less competent, more dangerous, less trustworthy, or less predictable) can, in 
turn, create prejudice leading to negative actions and discrimination (e.g., exclusion or withheld opportunities for work and residence; Ben-Zeev, Young, \& Corrigan, 2010; Crocker et al., 1998; Michaels et al., 2012; Wahl, 1999). As such, people with mental illness are, unfortunately, frequently subjected to public stigma and they are often labelled as being different, deviant, having undesirable characteristics, and seen as unpredictable, weird, dangerous, unstable, violent, and unintelligent (Gaebel, Baumann, \& Phil, 2003; Link, Phelan, Bresnahan, Stueve, \& Pescosolido, 1999). In fact, people with mental illness are probably among the most stigmatised, discredited, marginalised, and disadvantaged members of society (Johnstone, 2001), and, for people with mental illness and their families, the consequences of experiencing public stigma are, according to Johnstone (2011), "deeply dehumanising, culturally dispossessing, and radically alienating" (p. 200). Given its widespread stigmatisation, mental illness creates a two-pronged problem (Link, Struening, Rahav, Phelan, \& Nuttbrock, 1997) whereby people with mental illness not only have to deal with having mental illness but are also required to cope with and oppose public stigma and societal misunderstandings of mental illnesses (Link et al., 1997; Michaels et al., 2012). Findings by Link et al. (1997) also showed that stigmatisation by the general public continues to complicate the lives of people with mental illness even when treatment and therapies improve their functioning. The stigmatisation of people with mental illness can thus be as degrading as the symptoms of mental illness, leading to job discrimination, social exclusion, and conflicts within families (Feldman \& Crandall, 2007).

As stated above, stigmatisation not only affects those with a stigmatised condition but it may also affect the lives of family members, and mental illness stigma is no exception. Research has shown that family members of people with mental illness are frequently discredited and degraded because of their association with a family member with mental illness (Bos et al., 2013; Hebl \& Mannix, 2003; Pryor et al., 2012; Struening et al., 2001). Moreover, previous research has shown that family members of people with mental illness also face a two-pronged problem. With family members, this twopronged problem includes managing one's family member's mental illness, its symptoms, and that person's care needs, on the one hand, and coping with possible stigmatisation towards themselves and their family, including their family member with mental illness, on the other (Struening et al., 2001).

Public stigma aimed at people with mental illness has been studied extensively but stigma by association towards family members of people with mental illness has not. Therefore, the studies presented in this dissertation aim to advance our understanding of stigma by association as experienced by family members of people with mental illness by investigating the relationships between public stigma, stigma by association, psychological distress, perceived closeness, and quality of life. 


\section{Stigma by association and family members of people with mental illness}

Goffman (1963) theorised that public stigma not only affects stigmatised persons, but also people associated with them, and several studies have found that stigma spreads through social structure associations and relatively arbitrary associations created by proximity or similarity (Angermeyer, Schulze, \& Dietrich, 2003; Mehta \& Farina, 1988; Neuberg et al., 1994; Östman \& Kjellin, 2002; Pryor et al., 2012). This process through which friends, companions, caregivers, and family members of stigmatised people are discredited and stigmatised is, as stated previously, known as stigma by association (Bos et al., 2013; Hebl \& Mannix, 2003; Neuberg et al., 1994; Pryor et al., 2012; Stutterheim et al., 2011).

Stigma by association seems to emerge as cognitive representations and negative evaluations of a stigmatised person are linked to an associated person, such as a family member, friend, buddy, or another companion. The undesirable characteristics ascribed to the stigmatised person and the negative evaluations of this person are transferred to or projected upon the previously non-stigmatised companion rendering him or her tainted, degraded, or inferior (Kulik, Bainbridge, \& Cregan, 2008; Phelan, Bromet, \& Link, 1998; Pryor et al., 2012). Like public stigma, the process underlying stigma by association may be overt, but it may also be more subtle and less openly discriminatory (Duffy, Ganster, \& Pagon, 2002; Kulik et al., 2008).

Kinship relationships seem to provide a powerful conduit for stigma by association with stigma 'spilling' over from people with mental illness to their immediate family members (Pryor et al., 2012). This stigma by association can have serious negative effects on the lives of these family members (Angermeyer et al., 2003; Lanquetot, 1984; Lefley, 1989; Mehta \& Farina 1988; Neuberg et al., 1994; Östman \& Kjellin, 2002; Pryor et al., 2012; Zauszniewski, Bekhet, \& Suresky, 2008). Phelan et al. (1998) and other have outlined that stigma by association intensely and directly affects the mental health and quality of life of family members of people with mental illness, as much as primary experiences of public stigma can (Angermeyer et al., 2003; Östman \& Kjellin, 2002; Phelan et al., 1998; Tsang, Tam, Chan, \& Chang, 2003; Werner, Mittelman, Goldstein, \& Heinik, 2012). Furthermore, there is a body of research demonstrating that stigma by association can cause psychological distress, low self-esteem, shame, anger, hopelessness, anxiety, helplessness, and low quality of life for family members of people with mental illness (Byrne, 2000; Corrigan \& Miller, 2004; Kulik et al., 2008; Phelan et al., 1998). Psychological complaints such as restlessness, moodiness, agitation, irritability, and physical complaints such as insomnia, fatigue, inertia, and several physical aches and pains have been documented as symptoms of psychological distress and anxiety related to stigma by association (Angermeyer, Liebelt, \& Matschinger, 2001). Family members of people with mental illness who experienced stigma by association 
have reported experiencing negative treatment and social exclusion, avoiding social interactions, and spending energy and resources to conceal their relationship with their family members with mental illness as well (Larson \& Corrigan, 2008). Additionally, family members of people with mental illness have reported being blamed for the onset of their family members' mental illness, being held responsible for relapses, and being seen as incapable by members of their community (Corrigan et al., 2006).

Stigma by association is also known to affect how family members of people with mental illness view their family member with mental illness and these attitudes have been found to impact interpersonal relations and closeness within the family (Crowe \& Lyness, 2013; Kreisman \& Joy, 1974). Stigma by association therefore has a strong and long-lasting effect on the perceived closeness and relations between people with mental illness and their family members (Östman \& Kjellin, 2002).

\section{Family burden among families of people with mental illness}

Earlier research (Östman \& Hansson, 2000a, Östman \& Hansson 2000b; Östman \& Kjellin, 2002) has shown that it is not always easy for family members of people with mental illness to distinguish between experiences of stigma by association, family burden, and their impact. The impact and experiences of stigma by association and family burden might interact and entangle in their consequences (Lefley, 1989). Focusing on aspects of family burden as perceived by family members of people with mental illness may, therefore, be valuable in the context of increasing our knowledge of the experiences and consequences of stigma by association towards family members of people with mental illness.

Jenkins and Schumacher (1999) defined family burden as the presence of problems, social disruptions, behavioural difficulties, and adverse events due to a situation or illness within the family. Family burden is typically divided into two categories: objective burden and subjective burden (Schene, 1990). Objective burden comprises the actual, objective problems and difficulties associated with the situation or illness, whereas subjective burden relates to its psychological distress and consequences of the situation or illness (Lefley, 1989). Several studies (Jenkins \& Schumacher, 1999; Lefley, 1989; Östman \& Hansson, 2000b; Werner et al., 2012) have explored the relationship between the mental health condition of people with mental illness and the burden within their families. These studies tend to show that family members of people with mental illness experience a vast amount of family burden. There is also evidence of commonalities across families of people with mental illness. Such families frequently reported experiencing financial hardships, disturbances in household functioning, diminished social activities, and altered relationships with extended family and friends due to the time-consuming demands of care-giving (Lefley, 1989). The neglect 
of others in the family was also noted and attributed to an almost exclusive attentional focus on the person with mental illness (Lefley, 1989). Furthermore, family members of people with mental illness reported having to contend with deviant behaviour demonstrated by their family member with mental illness, manifest as mood swings, socially offensive behaviour, conflicts with neighbours, and patterns of losing or squandering money (Lefley, 1989). Additionally, family members of people with mental illness may suffer psychological distress and become even more stressed because of family burden, more specifically their inability to fulfil personal plans, their worries about the health condition and happiness of their family member with mental illness, their grief over the loss of the family member's premorbid personality, and their own grief for their diminished quality of life, failed aspirations, and lost developmental stages of learning (Lefley, 1987a).

Family burden is a subjective experience. Whereas objective burden primarily comprises the actual, objective problems and difficulties associated with a situation or illness, subjective burden is mainly examined in the context of psychological distress and consequences (Lefley, 1989). For this reason, several researchers have classified stigma by association as a dimension of subjective or psychosocial burden (De Boer, Mula, \& Sander, 2008; Sales, 2003; Schene, Tessler, \& Gamache, 1996). Taking another approach, Werner et al. (2012) reported stigma by association as a significant predictor of family burden. Evidently, it seems that there is no clear distinction between family burden and stigma by association (Östman \& Kjellin, 2002). However, there are various definitions and measures of family burden that do not include explicit references to stigma or stigma by association. Thus, one could contend that stigma by association is related to family burden, and their consequences may overlap to a certain extent, but the two constructs are unlikely to be identical. In this context, it is important to remember that the extent to which experiences and consequences of stigma by association and family burden overlap likely depends on how these concepts are defined and operationalised.

Regardless of their distinction or overlap, stigma by association and family burden both threaten the physical, psychological, emotional, and functional health of family members of people with mental illness. The way in which both are experienced and their impact also seems to differ between the various family members within the family of a person with mental illness (Angermeyer et al., 2003; Conde-Sala, Garre-Olmo, TurroGarriga, Vilalta-Franch, \& Lopez-Pousa, 2010; Phelan et al., 1998).

\section{Relevant family members' characteristics}

With regard to associations between possibly relevant characteristics of family members of people with mental illness like familial relationship, co-residence, gender, and 
the belief that mental illness is hereditary, on the one hand, and experiences of stigma by association and the consequences of those experiences for quality of their lives, on the other, previous studies are far from conclusive. Some studies have found characteristics like co-residence, gender, and the belief that mental illness is hereditary to be associated with increased levels of stigma by association and more detrimental consequences. Other studies have found no significant variation in the extent to which stigma by association is experienced or its consequence. The sections below describe, in more detail, these potentially relevant characteristics and why it may be important to consider them when studying stigma by association and its consequences.

\section{Familial relationship}

The first factor that may impact stigma by association is familial relationship. Different family members of people with mental illness are likely to experience different expressions and consequences of stigma by association. Generally, what the literature has shown is that parents and spouses experience more blame for the onset and continuation of the mental illness than others, while siblings and spouses are often held responsible for a lack of treatment adherence (Corrigan \& Miller, 2004). Östman and Kjellin (2002) also found that spouses of persons with mental illness had fewer social interactions.

\section{Co-residence}

Conde-Sala et al. (2010) found that living together with a family member with mental illness also had a significant effect on family burden and psychological distress among co-residing family members. Co-residence appeared to worsen family members' own mental health (Conde-Sala et al., 2010) in addition to affecting family members' possibilities of having social interactions with others as well (Östman \& Kjellin, 2002). Also, Lanquetot (1984) reported that co-residing with a family member with mental illness was associated with more shame, avoidance, social exclusion, rejection, and concealment of the relationship among family members of people with mental illness. Phelan et al. (1998), however, reported that people who live together with a family member with mental illness were less likely to conceal this relationship (Phelan et al., 1998).

\section{Gender}

Research has also shown that gender frequently, but not indisputably, impacts family members' experiences of stigma by association. Wirth and Bodenhausen (2009) reported that when a stigmatised condition was considered gender typical, blame was more likely to occur. Wahl and Harman (1989) reported that mothers more than others felt that they have been treated negatively by the general public. However, research by Chou and Mak (1998), Vuksic-Mihaljevic, Mandic, Barkic, Laufer, and Filakovic (1998), and Read and Harré (2001) failed to find significant gender differences in participants' experiences of stigma by association and its consequences. 
Belief that mental illness is hereditary

The extent to which a mental illness is considered hereditary has become an important item in the mental stigma discourse. Various researchers and advocates, including family members of people with mental illness who believe mental illnesses to be genetically determined, have expressed hopes that the recognition of the hereditary nature of some mental illnesses would reduce mental illness stigma; as such recognition would imply that people with mental illness are not responsible for their mental illness (Hoop, 2008; Phelan, Cruz-Rojas, \& Reiff, 2002). Others, however, have expressed concerns and a fear that this could exacerbate mental illness stigma, making the diagnostic label of mental illness 'stickier' and the potential for recovery smaller (Dietrich et al., 2004; Read \& Harré, 2001). A small amount of research has been conducted on the possible association between perceived experiences and consequences of stigma by association among family members of people with mental illness, on the one hand, and family members' belief in genetic factors and the hereditary nature of mental illnesses, on the other (Hoop, 2008; Phelan, 2002; Phelan, 2005; Phelan et al., 2002). Unfortunately, research results to date are ambiguous.

\section{Coping with stigma by association and family burden}

Experiences of stigma by association can be very stressful for family members of people with mental illness. These experiences often generate a need to monitor, manage, and adjust the stressful situation within the family. Consequently, family members are likely to make attempts to prevent, handle, or mitigate their experiences and the negative impact of stigma by association. They will make efforts to cope and alter the social and psychological impact of stigma by association such that it is less distressing (Stutterheim, 2011). However, stressors tend to only be perceived as stressful when they exceed one's resources and possibilities for coping (Miller \& Kaiser, 2001).

Coping can be defined as the process of conscious expenditure of resources and energy to regulate, minimise, or tolerate stressors. It comprises the management of emotional and cognitive processes and the behavioural reaction to the stress and burden originating from perceived experiences of stigma by association (Blum \& Silver, 2008). Coping with stigma by association is an ongoing process as family members are regularly confronted with new and changing social contexts, cognitive representations, and negative evaluations about people with mental illness, in general, and their family member with mental illness, in particular (Blum \& Silver, 2008). Generally, people mitigate experiences of stigma by association and make efforts to reduce its negative impact by employing various coping strategies, separately or simultaneously (Miller \& Kaiser, 2001). According to Lazarus and Folkman (1984), coping strategies can be problem-focused or emotion-focused (Miller \& Kaiser, 2001; Stutterheim et al., 2011). Problem-focused coping strategies include seeking instrumental support, seeking emotional 
support, humour, active coping, and planning and are aimed at managing the stressor and minimising its negative impact. Emotion-focused coping strategies include acceptance, positive reframing, self-blame, self-distraction, behavioural disengagement, venting, denial, substance use, and turning to religion and mainly focus on managing the negative emotions related to the stressor (Eaton, Davis, Hammond, Condon, \& McGee, 2011; Nydegger, Nydegger, \& Basile, 2011; Stutterheim et al., 2011; Tuncay, Musabak, Gok, \& Kutlu, 2008).

Whether problem-focused or emotion-focused, coping strategies seek to handle, prevent, or reduce a stressor, however, some coping strategies are adaptive, others are maladaptive, and still others are merely ineffective (Moore, Biegel, McMahon, 2011; Rippetoe \& Rogers, 1987). In general, problem-focused coping strategies are thought to be more adaptive and more likely to lead to positive effects in the long run than emotion-focused coping strategies that mainly reduce symptoms and emotions in the short term (Eaton et al., 2011; Hassan et al., 2006; Moore et al., 2011; Nydegger et al., 2011; Rippetoe \& Rogers, 1987).

\section{Aim and outline of the present dissertation}

This dissertation aims to explore the experiences of stigma by association among family members of people with mental illness, the consequences of stigma by association for family members of people with mental illness, and the ways in which family members of people with mental illness cope with the negative impact of stigma by association. To do this, the associations between public stigma, stigma by association, family burden, psychological distress, family closeness, and quality of life among family members of people with mental illness are investigated using a mixed methods approach (i.e., qualitative and quantitative methods; Adato, 2011). The strategies used by family members of people with mental illness to cope with their experiences of stigma by association are also examined, as are possible effects of potentially relevant characteristics, namely familial relationship, co-residence, gender, and the belief that mental illness is hereditary on these associations. The main research questions are therefore:

1. What have family members of people with mental illness experienced in terms of stigma by association?

2. What are the consequences of these perceived experiences of stigma by association among family members of people with mental illness?

3. Are familial relationship, co-residence, gender, and the belief that mental illness is hereditary associated with family members of people with mental illness' experiences of stigma by association and the consequences of stigma by association?

4. How can family members of people with mental illness cope with stigma by association and its consequences? 
The first part of this dissertation, chapters 2 and 3, focuses on the associations between public stigma, stigma by association, and the negative consequences of stigma by association, as well as the effect of relevant family members' characteristics on these associations. The second part of this dissertation, chapters 4 and 5 , investigates the strategies family members of people with mental illness use to prevent or mitigate experiences of stigma by association and family burden, and the impact of stigma by association and family burden on the lives of family members of people with mental illness.

In chapter 2 , the results of a quantitative cross-sectional survey study among 527 family members of people with mental illness are described. Associations between public stigma and experiences of stigma by association among family members of people with mental illness are explored, as are some of the consequences of stigma by association (i.e., psychological distress and perceived closeness). Also, the effects of the familial relationship between people with mental illness and their family members, as well as family members' belief that mental illness is hereditary are studied in detail.

Chapter 3 presents a qualitative study investigating the experiences of stigma by association among family members of people with mental illness and the impact of these experiences. More specifically, through semi-structured interviews with 23 people who are family members of people with mental illness, experiences and manifestations of stigma by association are explored alongside the social and psychological consequences of stigma by association. Additionally, this study explored the perceived impact of familial relationship, co-residence, and gender of the participant on experiences of stigma by association and its consequences.

In chapter 4 , more qualitative research on the strategies employed by family members to cope with experiences of stigma by association and its negative consequences is presented. Again, through semi-structured interviews with 23 family members of people with mental illness, experiences of stigma by association and family burden were explored. The results presented in this chapter focus specifically on how family members of people with mental illness cope with experiences of stigma by association, family burden, and the negative consequences of stigma by association and family burden.

In chapter 5, the ways in which family members of people with mental illness cope with stigma by association and family burden, and the negative effects of stigma by association and family burden, are investigated further. Relying on cross-sectional survey data from 503 family members of people with mental illness, this chapter first outlines the associations between stigma by association, family burden, psychological 
distress, and quality of life and then explores the mediating role of fourteen different coping strategies.

Finally, in chapter 6 , the main findings of the preceding chapters are summarised and a general discussion of these findings is provided. More specifically, the findings presented in the earlier chapters are discussed in the context of previous research and the current literature. This chapter also reflects on the methods used to conduct the research presented in this dissertation, the limitation of the findings, and the implications of the findings practice. Lastly, the chapter offers future research. 



\section{CHAPTER 2}

\section{EXPERIENCES OF STIGMA BY ASSOCIATION AMONG FAMILY MEMBERS OF PEOPLE WITH MENTAL ILLNESS}

This chapter has been published as:

Van der Sanden, R. L. M., Bos, A. E. R., Stutterheim, S. E., Pryor, J. B., \& Kok, G. (2013). Experiences of Stigma by Association among Family Members of People with Mental Illness. Rehabilitation Psychology, 58(1), 73-80. doi:10.1037/a0031752 


\begin{abstract}
The objective of this study was to investigate the relationships between public stigma, stigma by association, psychological distress, perceived closeness, perceived heredity, and the type of family relationship among family members of people with mental illness. In this cross-sectional survey, data from 527 family members of people with mental illness were analysed. Perceptions of public stigma were found to be positively related to stigma by association and stigma by association correlated with greater psychological distress and less perceived closeness. Stigma by association also mediated relationships between perceived public stigma and psychological distress, and between perceived public stigma and perceived closeness. Further, among participants who reported stigma by association, immediate family members showed lower levels of perceived closeness than extended family members. Also, the perceived heredity of mental illness was associated with perceptions of public stigma and psychological distress. The findings suggest that family members of people with mental illness could benefit from education on mental illnesses, their treatment, and the extent to which they are hereditary. Additionally, particular attention should be paid to the psychological needs that arise from being a caregiver of someone with mental illness.
\end{abstract}

Keywords: stigma by association, mental illness, family, mental health, heredity 


\section{IMPACT AND IMPLICATIONS}

Although mental illness stigma has been studied extensively, stigma by association among family members of people with mental illness has not. This cross-sectional survey aims to advance our understanding of stigma by association by investigating the relationships between perceived public stigma, stigma by association, psychological distress, and perceived closeness while considering the role of the perceived heredity of mental illnesses and family relationships.

The findings show that perceived public stigma and stigma by association contribute to psychological distress among family members of people with mental illness. They also indicate that believing mental illness is hereditary is associated with greater psychological distress. Further, lower levels of perceived closeness were reported among immediate family members who had experienced stigma by association than among extended family members who had experienced stigma by association.

Given their important role as caregivers and providers of social support, we suggest that interventions focus on aiding immediate family members of people with mental illness in their provision of social support by providing education regarding mental illnesses, their impact, their treatment, and the extent to which they are hereditary.

\section{INTRODUCTION}

Historically, people with mental illness have always been stigmatised. This stigma pervades writings from medieval to modern times. Thought to be possessed by demons in medieval times, and viewed as constitutionally weak, dangerous, and responsible for their own plight in recent decades, the diagnosis of a mental illness is almost always accompanied by stigma (Fink \& Tasman, 1992). The term stigma refers to a distinctive, discrediting characteristic that renders its bearer tainted, flawed, or inferior in the eyes of others (Bos et al., 2001; Crocker et al., 1998). The origin of stigmatisation lies in the cognitive representations of people who possess the stigmatised condition. These cognitive representations may trigger emotional and behavioural reactions from others that subsequently result in stigmatising behaviour such as avoidance, blaming, and exclusion (Bos, Schaalma, \& Pryor, 2008; Dijker \& Koomen, 2003). As such, stigma can occur as discrimination, rejection, and other negative social interactions (Black \& Miles, 2002; Corrigan et al., 2007). Mental illness stigma has a strong and enduring effect on psychological well-being (Link et al., 1997). Unfortunately, stigma continues to complicate the lives of people with mental illness, even as treatment improves their symptoms and functioning. 
Stigmatisation not only affects people with mental illness, but also their families. This phenomenon is known as stigma by association (Mehta \& Farina, 1988; Neuberg et al., 1994) or courtesy stigma (Angermeyer et al., 2003; Goffman, 1963). Stigma by association represents the process through which family members, friends and companions of stigmatised persons are devalued and discredited (Pryor et al., 2012). Although kinship relationships provide a powerful conduit for the spread of stigma from "marked" to "unmarked" persons, stigma can also spread through relatively arbitrary associations created by proximity or similarity (Neuberg et al., 1994; Pryor et al., 2012). Pryor et al., (2012) theorised that there are common affective, cognitive, and behavioural dimensions of reactions to having relatives or other social associates with stigmatised conditions. They found experiences of stigma by association to be strongly related to perceived public stigma (i.e., societal reactions to the stigma) and to predict poorer psychological well-being across various stigmatised conditions. Further, their findings suggest that experiencing stigma by association is associated with psychologically distancing oneself from a stigmatised relative. Reactions to stigma by association are thus not only personal reactions to the stigma itself, but also reactions to being connected to someone who possesses the stigma. Like the primary experience of stigma, stigma by association can directly affect the health and well-being of family members of people with mental illness (Angermeyer et al., 2003; Östman \& Kjellin, 2002; Phelan et al., 1998). Psychological complaints, such as brooding, inner unrest, and irritability, and physical complaints, such as insomnia, fatigue, and neck and shoulder pain, have been reported as symptoms of psychological distress caused by stigma by association (Angermeyer et al., 2001). Family members of people with mental illness have also reported avoiding social interactions, suffering social exclusion, and spending energy and resources to conceal their relationship to their family member as a result of stigma by association (Larson \& Corrigan, 2008).

Stigma by association can impact, and is impacted by, perceived closeness. Psychological closeness in relationships can be defined as the degree to which one includes the other in one's self (Aron \& Aron, 1986; Aron, Aron, Tudor, \& Nelson, 1991). Aron and colleagues have posited that, in a close relationship, one acts as if some or all aspects of the other (e.g., resources, perspectives, characteristics) are partially one's own. Closeness thus represents a vicarious sharing of the other's traits and abilities. However, being associated with a stigmatised person poses a dilemma. In the case of a family member with mental illness, one must either embrace the fate of that family member as a stigmatised person and identify with him or her, or one must reject sharing the discredit by avoiding or minimising the relationship (Kreisman \& Joy, 1974). At the same time, closeness permits one to see qualities other than the stigma. The closer the relationship, the less the stigma is perceived as defining the person. As such, closeness can yield reductions in stigma (Werner, Goldstein, \& Buchbinder, 2010). 
According to Corrigan et al. (2006), stigma by association may also vary by family role (e.g., parent, sibling, child). In fact, they reviewed ways in which various family roles were impacted by stigma by association and concluded that parents were blamed for causing their child's mental illness, siblings were blamed for not assuring that their family members adhere to treatment plans, and children were fearful of being "contaminated" with the mental illness of their parent.

Many persons with mental illness, their families, and clinicians, have expressed hopes that knowledge about the perceived heredity of mental illnesses will decrease stigma by demonstrating that mental illness is primarily biologically determined and that individuals should not be blamed for their illness. However, some researchers contend that knowledge of the genetics of mental illness worsens stigma by making the diagnostic label of mental illness "stickier" (Hoop, 2008; Phelan, 2005; Phelan et al, 2002; Read \& Harré, 2001). As such, perceived heredity allows social connections to be fortified by biological ones. This view is supported by work indicating that attributions to heredity enhance negative reactions to people with mental illness (Dietrich et al., 2004) and to members of their families (Koschade \& Lynd-Stevenson, 2011).

Experiences of stigma by association among family members of people with mental illness have received comparatively little attention in empirical research. The present study therefore examined the relationships between perceived public stigma, stigma by association, psychological distress, perceived closeness, perceived heredity, and the type of family relationship (immediate vs. extended family) in a large sample of family members of people with mental illness in the Netherlands. We hypothesised, first, that perceived public stigma would be positively related to stigma by association and, second, that stigma by association would be positively related to psychological distress and negatively related to perceived closeness. We further hypothesised positive associations between perceived heredity on the one hand, and perceived public stigma, stigma by association, and psychological distress on the other hand, exist. In addition, we posited that the type of family relationship (immediate vs. extended) would moderate the relationships between stigma by association and psychological distress and between stigma by association and perceived closeness. More specifically, we expected stigma by association to be associated with higher levels of psychological distress and lower levels of perceived closeness more in immediate family members than in extended family members. 


\section{METHOD}

\section{Participants and procedure}

In November 2010, family members of people with mental illness in the Netherlands were recruited from an online panel $(N=13,196)$ through an email that asked whether they had a family member with mental illness who was receiving or had received mental health care and if they were willing to participate in a survey on this topic. Approximately 6,800 panel members responded to this email, and we confirmed that $39 \%(n=$ 2,654 ) had a family member with mental illness who was receiving or had received mental health care. A random sample of 666 cases drawn from these 2,654 panel members meeting the inclusion criteria were subsequently invited by email to participate in the survey and a reminder was sent 4 days after the initial invitation. Of the 666 invitees, 527 completed the survey, yielding a response rate of $79.1 \%$. Informed consent was obtained, and participants were given points that could be exchanged for discount coupons on survey completion. The study was approved by the ethics committee at Maastricht University's Faculty of Psychology and Neuroscience.

Participant characteristics are displayed in Table 2.1. In short, 211 were men and 316 women. Ages ranged from $18-85$ years $(M=43.4, S D=14.9)$. In terms of educational attainment, $48.0 \%$ had a low (i.e., elementary school or lower vocational training), $27.7 \%$ a moderate (i.e., secondary or midlevel vocational training), and $24.3 \%$ a high (i.e., college or university) level of education. The relationship of participants to their family member with mental illness varied widely: $17.3 \%$ were parents, $21.3 \%$ were children, $16.3 \%$ were siblings, $12.1 \%$ were spouses, $9.6 \%$ were in-laws, and $23.4 \%$ had some other family relationship. 
Table 2.1 Demographic and Background Characteristics of Sample ( $N=527)$

\begin{tabular}{|c|c|}
\hline Variable & Percentage (\%) \\
\hline \multicolumn{2}{|l|}{ Family relationship: } \\
\hline Spouse & 12.1 \\
\hline Child & 21.3 \\
\hline Parent & 17.3 \\
\hline Sibling & 16.3 \\
\hline In-laws & 9.6 \\
\hline Other & 23.4 \\
\hline \multicolumn{2}{|l|}{ Gender } \\
\hline Male & 40.0 \\
\hline Female & 60.0 \\
\hline \multicolumn{2}{|l|}{ Age } \\
\hline Years [mean (SD)] & 43.4 (14.9) \\
\hline Range (min-max) & $18-85$ \\
\hline \multicolumn{2}{|l|}{ Level of Education $^{1}$} \\
\hline Low & 48.0 \\
\hline Moderate & 27.7 \\
\hline High & 24.3 \\
\hline \multicolumn{2}{|l|}{ Marital status } \\
\hline Single & 18.1 \\
\hline Common-law & 17.6 \\
\hline Married & 56.9 \\
\hline Divorced & 5.7 \\
\hline Widowed & 1.7 \\
\hline \multicolumn{2}{|l|}{ Ethnicity } \\
\hline Dutch & 97.5 \\
\hline Other & 2.5 \\
\hline
\end{tabular}

\section{Measures}

The type of mental illness participants' family member had was assessed by asking participants to select their family member's mental illness(es) from the following list: schizophrenia or psychotic disorder, eating disorder, depressive disorder, addiction, personality disorder, attention deficit hyperactivity disorder (ADHD), autism, anxiety disorder, bipolar or other mood disorder, or another mental illness. More than one disorder could be selected.

\footnotetext{
${ }^{1}$ Low = elementary school or lower vocational training; moderate = secondary school or mid-level vocational training; high = college or university
} 
Perceived public stigma was assessed using the 18-item Public Stigma Scale (Pryor et al., 2012; $\alpha=.84$ ) in which items represent constructs examined in prior studies of public stigma (i.e., Corrigan, Markowitz, Watson, Rowan, \& Kubiak, 2003; Crandall \& Moriarty, 1995; Feldman \& Crandall, 2007; Link, Cullen, Struening, Shrout, \& Dohrenwend, 1989; Link et al., 1999; Vogel, Wade, \& Hackler, 2007). On each item, participants were asked to rate the degree to which they thought most people would react as described to someone with mental illness, on a 5-point scale ranging from 1 (strongly disagree) to 5 (strongly agree). Cronbach's alpha was .83.

Stigma by association was measured using the 28-item Stigma-by-Association Scale (Pryor et al., 2012; $\alpha=.94$ ) that measures participants' cognitive, emotional, and behavioural reactions to being related to someone with a stigmatised condition. Items were rated on a 9-point scale, ranging from 1 (strongly disagree) to 9 (strongly agree). Cronbach's alpha was .90.

Psychological distress was assessed using the 18-item Mental Health Inventory (MHI) ( $\alpha=.94$; Veit \& Ware, 1983). The MHI measures depression (e.g., "Have you felt downhearted or blue?"), anxiety (e.g., "Have you been a nervous person?"), positive affect (e.g., "Has your daily life been full of things that you been in firm control of your behaviour, thoughts, emotions, feelings?") over the 4 weeks prior to administration by having participants score items on a 6-point scale, ranging from 1 (none of the time) to 6 (all of the time). A higher score is indicative of greater psychological distress. The $\mathrm{MHI}$ is a valid and reliable measure of mental health that has been used extensively in both clinical and nonclinical samples (Cassileth et al., 1984; Heubeck \& Neill, 2000; Rosenthal et al., 1991; Veit \& Ware, 1983). It was also used previously to examine the impact of stigma on psychological distress (Stutterheim et al., 2009; Stutterheim et al., 2011). Cronbach's alpha was .94.

Perceived closeness was assessed using the single-item Inclusion of Other in the Self Scale (IOS; Aron, Aron, \& Smollan, 1992). In the administration of this scale, seven diagrams are presented. Each diagram depicts two circles, one labelled self and the other labelled other. The two circles have varying degrees of overlap, from no overlap to almost complete overlap. Participants are asked to pick the diagram that best represents their relationship to the person of interest. In our study, this was their family member with mental illness. Greater overlap represents more perceived closeness. The scale, widely used in relationship research, has demonstrated strong predictive and construct validity as a general measure of closeness. In fact, Aron et al. (1992) reported an alternate-form reliability of .95 and a test-retest reliability over 2 weeks of .85 (Lewandowski, Aron, Bassis, \& Kunak, 2006). The IOS has also been found to correlate significantly with other popular closeness measures such as the Relation Closeness 
Inventory $(r=.22)$, the Subjective Closeness Inventory $(r=.34)$, and the Sternberg Intimacy Scale ( $r=.45$ ) (Agnew, Loving, Le, \& Goodfriend, 2004).

Perceived heredity was measured by a single item, namely, "To what extent do you believe that your family member's mental illness is genetically determined?" This item was scored on a 5-point scale, ranging from 1 (not at all) to5 (very much). A higher score was considered indicative of greater perceived heredity.

Demographic variables including age, sex, educational attainment, marital status, ethnicity, and religious orientation were also assessed, as was the type of family relationship between participants and their family member with mental illness (i.e., mother, father, brother, sister, son, daughter, spouse, uncle, aunt, cousin, grandparent, or inlaw).

\section{RESULTS}

First, descriptive statistics for the various categories of mental illness were generated. The most frequently reported mental illness among family members was depression $44.0 \%(n=232)$. ADHD by $13.5 \%(n=71)$, personality disorder by $12.0 \%(n=63)$, schizophrenia or psychotic disorder by $9.3 \%(n=49)$, addiction by $8.3 \%(n=44)$, and eating disorder by $5.5 \%(n=29)$. An additional $6.8 \%(n=36)$ indicated that their family member had a mental illness other than the abovementioned disorders. Note that participants were allowed to select more than one mental disorder. As such, the sum of the percentages exceeds $100 \%$.

Next, we compared our participants' scores on psychological distress (MHI) and perceived closeness (IOS) to scores of samples in earlier studies. When comparing scores on the $\mathrm{MHI}$, for which no clinical cut-off scores have been established, we first compared our sample's scores to normative data collected from the general population by Manne, Ostroff, Fox, Grana, and Winkel (2009) and by Stewart, Sherbourne, and Hays (1992). We compared mean scores with a $t$ test and found the mean in our sample ( $M$ $=50.30, S D=14.72)$ to be significantly higher than the normative mean $(M=23.00, S D$ $=19.20)$ in the general population, $t(526)=13.02, p<.001$. We then, in accordance with prior studies suggesting that scores with a standard deviation greater than 1.5 above the normative mean should be used as a clinical cut-off for psychological distress (Norton et al., 2004), categorised participants' scores as low $(<0.5$ SD above the normative mean), moderate (0.5 - 1.49 SD above the normative mean), and high (> 1.5 $S D$ above the normative mean), and found that $12 \%$ of our sample had scores indicating low psychological distress, $46 \%$ had scores suggesting moderate psychological distress, and $42 \%$ percent had scores indicating high levels of psychological distress. 
The percentage of participants with high levels of psychological distress was significantly higher than that of the general population. Similarly, we compared our sample's scores on the IOS to normative data derived from a study conducted by Aron et al. (1992), in which undergraduate students in the United States were asked to describe their relationship with their closest other. We compared mean scores in our sample $(M$ $=3.86, S D=2.11)$ to those of Aron et al.'s sample $(M=4.74, S D=1.48)$, while acknowledging that the samples may be qualitatively different, and found perceived closeness to be significantly lower in our sample, $t(526)=9.54, p<.001$.

We subsequently examined the bivariate relationships between the primary variables in our study and found perceived public stigma to correlate positively with stigma by association, $r=.29, p<.001$, and psychological distress, $r=.09, p<.05$, and correlate negatively with perceived closeness, $r=-.10, p<.05$. Stigma by association correlated positively with psychological distress, $r=.15, p<.001$ and negatively with perceived closeness, $r=-.16, p<.001$. These correlations, along with the means and standard deviations for the primary study variables, are presented in Table 2.2. Regression analyses, in which we controlled for demographic variables, showed similar results.

Table 2.2 Means, standard deviations, and intercorrelations for main study variables

\begin{tabular}{lllllll}
\hline Variable & $M$ & $S D$ & 1 & 2 & 3 & 4 \\
\hline Perceived public stigma & 3.51 & .48 & -- & $.29 * * *$ & $.09^{*}$ & $-.10^{*}$ \\
Stigma by association & 1.93 & 1.06 & -- & $.15^{* * *}$ & $-.16^{* * *}$ \\
Psychological distress & 2.79 & .82 & & -- & .06 \\
Perceived closeness & 4.00 & .21 & & & -- \\
\hline
\end{tabular}

${ }^{*} p<.05,{ }^{* *} p<.01,{ }^{* * *} p<.001$

Next, we conducted mediation analyses in accordance with Baron and Kenny (1986). We found stigma by association to fully mediate the relationship between perceived public stigma and psychological distress, $z=3.02$, SE $=.02, p<.01$ (see Figure 2.1). Stigma by association also fully mediated the relationship between perceived public stigma and perceived closeness, $z=-3.31, S E=.06, p<.001$ (see Figure 2.2 ), thus suggesting that, as perceived public stigma increases, so does stigma by association, but that experiences of stigma by association are more proximally related to psychological distress and closeness. 
Perceived public stigma $\stackrel{.29 * * *}{\longrightarrow}$ Stigma by association $\stackrel{.15^{* * *}}{\longrightarrow}$ Psychological distress

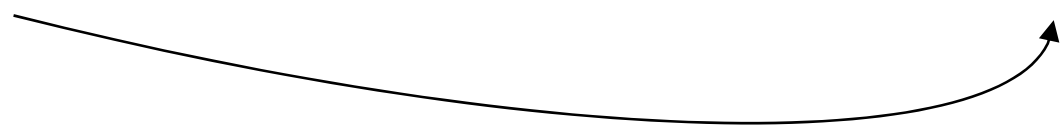

$.05(.09 *)$

$* p<.05, * * p<.01, * * * p<.001$

Figure 2.1 Mediation analyses whereby stigma by association mediates the relationship between perceived public stigma and psychological distress
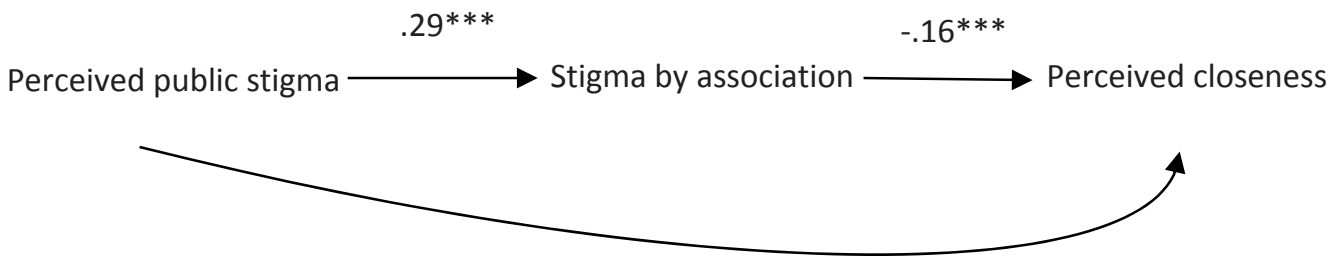

$-.06(-.10 *)$

$* p<.05, * * p<.01, * * * p<.001$

Figure 2.2 Mediation analyses whereby stigma by association mediates the relationship between perceived public stigma and perceived closeness

Following the mediation analyses, we examined, via correlation and regression analyses, the relationship between perceived heredity and the other study variables. Correlation analyses showed perceived heredity to correlate positively to perceived public stigma, $r=.12, p<.01$, and psychological distress, $r=.18, p<.001$, but no significant relationship to stigma by association was found. Similar findings were yielded in regression analyses. Perceived heredity was associated with perceived public stigma, $F(1$, $525)=7.49, \beta=.12, p<.01$, and psychological distress, $F(1,525)=17.61, \beta=.18, p<$ .001 , but not with stigma by association.

Lastly, we investigated how the family relationship between the participant and his or her relative with mental illness related to the other study variables. To do this, we dichotomised the sample into immediate family members of people with mental illness (parent, sibling, or child; $n=290$ ) and extended family members of people with mental illness (not a parent, sibling, or child; $n=237$ ). We then ran one-way analyses 
of variance to test for differences in stigma by association, psychological distress, and perceived closeness, and found that only perceived closeness differed significantly across immediate $(M=4.11, S D=2.04)$ and extended family $(M=3.57, S D=2.17), F(1$, $525)=8.91, p<.01, \eta^{2}=.02$. Next, we explored whether the type of family relationship moderated the relationships between stigma by association and psychological distress and between stigma by association and perceived closeness. In both analyses, we first entered the independent (stigma by association) and moderating (type of family relationship) variables (Step 1), then entered these variables into a regression equation (Step 2), and, lastly, we added an interaction term representing the product of stigma by association and the type of family relationship to the analyses (Step 3). We found no significant moderating effect in the psychological distress analyses, but we did find a significant moderating effect in the perceived closeness analyses, $F(1,525)=7.13, p$ $<.01$, and we found the interaction between stigma by association and the type of family relationship to be significant, $\beta=.25, p<.01$. Simple slopes analyses were then conducted to further investigate this significant interaction (Aiken \& West, 1991). These analyses showed that when the family relationship was immediate, stigma by association correlated negatively to perceived closeness, $\beta=.27, p<.001$. However, when the relationship was extended, stigma by association did not correlate significantly to perceived closeness, $\beta=.05, p<.46$. We followed this with linear regression analyses to determine which specific relationships were significant in the association between stigma by association and perceived closeness; we found that being a parent, $F(1,109)=5.15, \beta=.21, p<.05$, a sibling, $F(1,86)=4.47, \beta=.22, p<.05$, or a child, $F(1$, $89)=7.15, \beta=.27, p<.01$, of someone with mental illness is significant. Similar linear regression analyses were also conducted for association between stigma by association and psychological distress. They showed that being a parent, $F(1,109)=4.37, \beta=$ $.20, p<.05$, or child, $F(1,89)=4.09, \beta=.21, p<.05$, of someone with mental illness is significant.

\section{DISCUSSION}

This study is, to our knowledge, the first to examine the relationships between the perceived public stigma of mental illness, stigma by association, psychological distress, the perceived closeness to one's family member with mental illness, the perceived heredity of mental illness, and the role of family relationship in a large sample of family members of people with mental illness. Our results show that the perceived public stigma of mental illness stigma correlates positively to experiencing stigma by association. This supports the notion that stigma by association is, at least in part, derived from an awareness of the general societal reactions to a stigma. Other research has shown that internalised stigma, often called self-stigma, also correlates with perceived public stigma (Bathje \& Pryor, 2011; Vogel et al., 2007). The results also show stigma 
by association to correlate positively to psychological distress and negatively to perceived closeness. This supports the contention that stigma by association may motivate family members of people with mental illness to psychologically distance themselves from a relative with mental illness, perhaps in an effort to detach oneself from the stigma carried by their family member. This is in line with studies that have examined the experiences of people whose family members have serious mental illnesses (Östman \& Kjellin, 2002). At the same time, it is important to recognise that psychological distress and perceived closeness within families of people with mental illness are complex processes. In fact, as previously outlined by Schene (1990), the lives and social relationships of family members of people with mental illness can be substantially disrupted or diminished as a result of providing intensive care to a family member with mental illness or because of economic or practical burdens that result from having a relative with mental illness. This may, in part, explain why the participants in our study experienced higher levels of psychological distress and diminished perceived closeness than comparison samples derived from the general population. Another potential explanation is that family members of people with mental illness are more sensitive to negative experiences in their social environment, including stigma by association.

We also found stigma by association to mediate the relationships between perceived public stigma and psychological distress and between perceived public stigma and perceived closeness. This may imply that the psychological impact of stigma by association on family members is not solely a reaction to the stigma itself, but also a reaction to being connected to someone with the stigma of mental illness. It also supports the idea that stigma by association is related to, but to some extent distinct from, family members' sense of public stigma.

Our finding that the perceived heredity of mental illness is associated with both perceived public stigma and psychological distress supports the work of Phelan et al. (2002), who found that people who thought that mental illness was attributable to genes were less likely to think mental illness could be improved with appropriate help. In their study, they concluded that the "stickiness" of a mental illness label could be exacerbated by a genetic attribution. In our study, the perceived heredity of mental illness was not related to stigma by association, but was, nonetheless, associated with psychological distress. This seems to suggest that when mental illness is considered genetically determined, uncertainty about one's health may ensue and concerns regarding latent mental illness may result. We believe that being confronted with a family members' mental illness and stigma may stimulate fear and uncertainty (Hoop, 2008), which, in turn, may contribute to psychological distress. An alternative explanation is that family members of people with mental illness who believe their family member's mental illness is hereditary find that their family member is neither responsible nor blameworthy for his or her mental illness (Hoop, 2008). This could not only 
make family members of people with mental illness more sensitive to public stigma, but could also make them identify public stigma as a social injustice (Corrigan, Watson, Byrne, \& Davis, 2005; Johnstone, 2001), which, in turn, might be associated with higher levels of distress.

We also found that immediate family members (i.e., parents, siblings, and children of people with mental illness) who reported experiences of stigma by association had lower levels of perceived closeness than extended family members who reported experiences of stigma by association. This could be because immediate family members, who are often directly and on a day-to-day basis confronted with their family members' mental illness, the corresponding stigma, and its consequences, seek to distance themselves from their family members' stigma, traits, and abilities. They may even conceal their relationship to their family member with mental illness to avoid stigma by association. This too is in accordance with work conducted by Phelan et al. (1998), who, in a study of perceptions of, and reactions to, stigma among relatives of psychiatric patients, found that relatives actively forestall avoidance by concealing their relative's mental illness from others. In addition, in Phelan et al.'s (1998) study, parents and children of people with mental illness who reported experiences of stigma by association also had higher levels of psychological distress than other family members. In earlier studies, psychological distress and burden have been found to be greater when patient contact is extensive or when patients live with their families (Burke, 2003; Robinson, Rodgers, \& Butterworth, 2008). Another characteristic of family relationships that may contribute to psychological distress is the inevitable responsibility (parents) and dependency (children) of the actors within these relationships. Possibly, psychological distress in parents and children of people with mental illness is the result of feelings of loss, sadness, the frustration caused by changing relationships and/or grief for the loss of person's former personality or previous family lifestyle (Shah, Wadoo, \& Latoo, 2010).

\section{Study strengths and limitations}

Our study has strengths and limitations. Our primary strength is our large representative sample that allowed for the examination of stigma by association and other related constructs across a broad spectrum of mental illnesses. The cross-sectional nature of our data is, however, a limitation because no conclusions about causality can be drawn. We suggest that future research adopt a longitudinal design. Our reliance on self-reported data is also a possible limitation. However, the potential bias that results from self-reported data was minimised insofar as was possible by using previously validated measures and by assuring participants that data would be processed anonymously. Further, it is possible that the external validity of the study was compromised by our use of an Internet panel. Using an Internet panel may have led to particularly 
motivated individuals participating more than less motivated individuals. Additionally, because participants were selected from a pool of family members of people with mental illness who had received treatment, the findings can only be generalised to families of people with mental illness who have received treatment. As such, it is possible that the participants in our study had previously been offered support by mental health institutions, social workers, and/or advocacy groups, thus enabling them to better cope with public stigma and stigma by association. If this were the case, stigma by association may be underestimated in our study. Another possible limitation is the fact that we did not document whether participants lived together with their family member with mental illness. This should be documented in future studies, because it may influence the intensity and impact of the interactions between people with mental illness and their family members. Future research should also investigate stigma by association qualitatively such that the various relationships and the context to which stigma by association is experienced can be explicated and given context. Additionally, we recommend linking data on perceived stigma among people with mental illness to data on stigma by association among their family members.

The findings of our study have implications for both practice and theory. Our finding that the perceived heredity of mental illnesses is positively associated with perceived public stigma and psychological distress suggests that presumed heredity of mental illness may negatively impact not only people with mental illness but also their families' members. Our findings also point to the need for education about the degree to which a family member's mental illness is hereditary and the potential impact this may have on one's own mental health. In thinking about these associations, the whole family should be considered (Phelan et al., 2002). Information on recognising mental health problems and local sources of treatment and support should be made readily available to family members of people with mental illness and attention should be paid to the needs of family members of people with mental illness as caregivers. More specifically, families need support, contact, education, understanding, empathy, and respite when exhausted as a result of caregiving (Robinson et al., 2008; Corrigan \& Miller, 2004). Health care professionals can play an important role in helping family members of people with mental illness enhance existing coping skills. They can also facilitate the development of new ones (Shah et al., 2010). Additionally, we suggest that health care professionals involve family members of people with mental illness in their treatment and encourage family members of people with mental illness to participate in psychoeducation because this may reduce perceptions of stigma by association and lessen fears of genetic contamination. We also recommend, given our finding that the type of family relationship influences the association between stigma by association and perceived closeness, that social support provision within immediate families of people with mental illness be promoted. In fact, we recommend openness about family members' mental illness within the family and selective disclosure to others because this 
likely stimulates social support provision and lessens stigmatising responses (Bos et al., 2009).

In terms of theory, our findings contribute to the literature on mental illness stigma and, in particular, stigma by association among family members of people with mental illness. Bos, Pryor, Reeder, and Stutterheim (2013) have argued that empirical research on the interrelatedness of stigma manifestations is essential. Our study demonstrates that stigma by association mediates the relationship between perceived public stigma and psychological distress among family members of people with mental illness. Our findings also contribute substantially to the debate on the impact of thinking that mental illness is hereditary. They support the contention that perceived heredity and type of family relationship are linked to perceived public stigma, stigma by association, and psychological well-being.

\section{Conclusion}

This study has investigated and thereby contributed to our understanding of the relationships between perceived public stigma, stigma by association, psychological distress, and perceived closeness. It has also added to the current literature by demonstrating that both the perceived heredity of mental illnesses and the type of family relationship are related to perceptions of stigma, stigma by association, psychological distress, and perceived closeness. 


\section{CHAPTER 3}

\section{STIGMA BY ASSOCIATION AMONG FAMILY MEMBERS OF PEOPLE WITH A MENTAL ILLNESS: A QUALITATIVE ANALYSIS}

This chapter has been published as:

Van der Sanden, R. L. M., Bos, A. E. R, Stutterheim, S. E., Pryor, J. B. \& Kok, G. (2015). Stigma by association among family members of people with mental illness: A qualitative analysis. Journal of Community and Applied Social Psychology, 25. doi:10.1002/casp.2221 


\begin{abstract}
People with mental illness are not the sole recipients of stigmatisation; their immediate family members may be subjected to stigma by association. Through semistructured interviews, we investigated experiences of stigma by association among 23 immediate family members of people with mental illness. Participants reported experiencing stigma by association from community members, mental health professionals, and civil servants. Familial relationship, co-residence, and the gender of participants appeared to play a role in their stigma experiences; parents and spouses reported different manifestations of stigma by association than siblings and children, participants who lived together with their family member with mental illness reported increased experiences of stigma by association, and in contrast to male participants, female participants reported others thinking they are overprotective and as such perpetuated, maintained, or sustained their family members' mental illness. The relevance of these factors points to the need for tailored education and emotional support provision to family members of people with mental illness. Moreover, in-service training for mental health professionals should include the development of relevant social skills that enable the recognition of familial relationships and roles, and family members' fears, concerns, and problems.
\end{abstract}

Keywords: stigma by association, mental illness, familial relationship, co-residence, gender 
People with mental illness are more likely to be unemployed, have less income, and have less social support (Link \& Cullen, 1990). Labelling theory attributes these deficits partly to the stigma of mental illness (Link et al., 1989). The term stigma originally refers to a discrediting characteristic that renders someone flawed, degraded, or inferior in the eyes of others (Crocker et al., 1998). Nowadays, stigma is seen not as a physical mark or characteristic but rather as an attribute that results in widespread social disapproval (Bos et al., 2013). Dovidio et al. (2000) emphasized that definitions of stigma mostly comprise two components: difference and devaluation. To trigger stigmatising behaviour, the stigma should be noticeable and devalue one's social status. This may result in avoidance, blaming, exclusion, and other negative social interactions (Bos, et al., 2009).

Stigma may also "spill over" from people with a stigmatised condition to people associated with them, for example, family and friends. This is known as courtesy stigma, family stigma, or stigma by association (Bos et al., 2013; Corrigan et al., 2006; Goffman, 1963; Lefley, 1989; Pryor et al., 2012). Stigma by association can lead to social exclusion, avoidance of social interactions, negative treatment, and the expenditure of energy and resources to conceal one's familial relationship with a stigmatised person (Larson \& Corrigan, 2008). Studies have demonstrated that stigma by association can also affect the well-being of family members of people with a stigmatised condition like mental illness, resulting in psychological and physical health complaints (Angermeyer, et al., 2003; Östman \& Kjellin, 2002; Van der Sanden, Bos, Stutterheim, Pryor, \& Kok, 2013).

Research has shown that when the onset or continuation of a stigmatised condition like mental illness is perceived to be something over which one has control, others are more likely to view one as personally responsible for the condition, what may result in negative emotional reactions and subsequent stigmatisation (Bos et al., 2008; Bos et al., 2009; Phelan et al., 1998; Van der Sanden, 2006; Weiner, Perry, \& Magnusson, 1988). Corrigan and Miller (2004) have shown that this applies not only to people with mental illness but to their family members as well.

However, the term "family" implies a variety of familial relationships (e.g., parents, spouses, children, siblings) and responsibilities that differ across family members (Corrigan \& Miller, 2004; Eldering, 2006). As such, it is likely that different family members experience different expressions of stigma by association. Corrigan and Miller (2004) found that spouses and parents are frequently blamed for the onset and continuation of their family members' mental illness, while siblings and spouses are frequently held responsible for a family member's lack of treatment adherence, and children of people with mental illness often fear becoming 'contaminated' by their parents' mental illness. Mehta and Farina (1988) and Farina (2000), in their investigations of why com- 
munity members extend mental illness stigma to other, previously non-stigmatised family members, found that family members are stigmatised because people who appear together in public seem alike, and someone who voluntarily chooses to associate with a stigmatised person must be deviant as well. Furthermore, research has shown that also gender is regularly, however not indisputably, a factor in stigma experiences of people with mental illness (Farina, 1981; Östman \& Kjellin, 2002; Wirth \& Bodenhausen, 2009). In fact, Wirth and Bodenhausen (2009) demonstrated that when stigmatised conditions, symptoms, and behaviour are considered gender typical, blaming is more likely.

In this qualitative study, we comprehensively explored the experiences of stigma by association among immediate family members of people with mental illness, while paying particular attention to the characteristics of familial relationship, co-residence, and gender in order to better understand how experiences of stigma by association affect various immediate family members of people with mental illness and in order to enable the identification of adequate tailored support and education for family members of people with mental illness.

\section{METHOD}

\section{Participants and procedure}

After approval by the Ethics Committee at Maastricht University's Faculty of Psychology and Neurosciences, 23 immediate family members (i.e., spouses, children, siblings, and parents) of people with mental illness were recruited for face-to-face, semistructured interviews. Nineteen participants were recruited through announcements distributed by Dutch mental health self-help support groups and professionally operated support groups. Four participants were recruited directly by the first author. The self-help support groups are characterised by a desire to overcome difficulties associated with family members' mental illness and increase well-being and quality of life through bottom-up support and information seeking, empowerment, and the creation of communities of family members of people with mental illness. The professionally operated support groups are groups facilitated by mental health professionals with guided conversations that seek to allow family members of people with mental illness to share their experiences, acquire relevant information, establish social networks, and influence public opinion and policy. Demographic and background characteristics for all participants are displayed in Table 3.1. 
Table 3.1 Demographic and Background Characteristics of Sample $(N=23)$

\begin{tabular}{|c|c|}
\hline Variable & Percentage (\%) \\
\hline \multicolumn{2}{|l|}{ Sex } \\
\hline Male & 47.8 \\
\hline Female & 52.2 \\
\hline \multicolumn{2}{|l|}{ Age } \\
\hline Years (mean (SD)) & $44.3(12.6)$ \\
\hline Range (min-max) & $25-64$ \\
\hline \multicolumn{2}{|l|}{ Marital status } \\
\hline Single & 13.1 \\
\hline Married & 52.1 \\
\hline Divorced & 21.7 \\
\hline Widowed & 13.1 \\
\hline \multicolumn{2}{|l|}{ Family relationship } \\
\hline Spouse & 20.7 \\
\hline Child & 20.7 \\
\hline Parent & 27.5 \\
\hline Sibling & 31.1 \\
\hline \multicolumn{2}{|l|}{ Family members' mental illness ${ }^{2}$} \\
\hline Depressive, bipolar, or other mood disorders & 52.2 \\
\hline Personality disorder & 17.4 \\
\hline $\mathrm{ADHD} / \mathrm{ADD}^{3}$ & 17.4 \\
\hline Dissociative disorder & 13.0 \\
\hline Autism & 13.0 \\
\hline Schizophrenia or psychotic disorder & 8.7 \\
\hline Addiction & 4.3 \\
\hline
\end{tabular}

\footnotetext{
${ }^{2}$ Because some family members were diagnosed for more than one mental illness, the percentage of illnesses exceeds $100 \%$.

${ }^{3} \mathrm{ADHD}=$ attention deficit disorder with hyperactivity; $\mathrm{ADD}=$ attention deficit disorder
} 
An overview on familial relationship, co-residence, and gender per participant is provided in Table 3.2 .

Table 3.2 Overview on Familial Relationship, Co-residence, and Gender per Participant

\begin{tabular}{|c|c|c|c|c|c|}
\hline Participant ID & Name* & Age** $^{* *}$ & Familial relationship & Coresidence & Gender \\
\hline 1 & Renske & $56-60$ & Mother / Sister & No & Female \\
\hline 2 & Suzanne & $56-60$ & Mother & No & Female \\
\hline 3 & Emma & $56-60$ & Mother & No & Female \\
\hline 4 & Natasja & $56-60$ & Mother / Spouse & Yes & Female \\
\hline 5 & Anja & $61-65$ & Mother / Spouse & No & Female \\
\hline 6 & Karen & $56-60$ & Mother / Daughter & No & Female \\
\hline 7 & Hans & $56-60$ & Father / Spouse & No & Male \\
\hline 8 & Bas & $46-50$ & Father & Yes & Male \\
\hline 9 & Roy & $41-45$ & Spouse & Yes & Male \\
\hline 10 & Inge & $51-55$ & Spouse & No & Female \\
\hline 11 & Dick & $51-55$ & Spouse & Yes & Male \\
\hline 12 & Wendy & $56-60$ & Sister & No & Female \\
\hline 13 & Celia & $46-50$ & Sister & No & Female \\
\hline 14 & Liza & $26-30$ & Sister & No & Female \\
\hline 15 & Ria & $41-45$ & Sister & No & Female \\
\hline 16 & Wil & $61-65$ & Brother & No & Male \\
\hline 17 & Joep & $41-45$ & Brother & No & Male \\
\hline 18 & Theo & $26-30$ & Brother & No & Male \\
\hline 19 & Piet & $21-25$ & Brother / Son & No & Male \\
\hline 20 & Erik & $36-40$ & Son & No & Male \\
\hline 21 & Max & $36-40$ & Son & Yes & Male \\
\hline 22 & Joey & $26-30$ & Son & No & Male \\
\hline 23 & Chris & $56-60$ & Son & Yes & Male \\
\hline
\end{tabular}

*Pseudonym **categorised

Once recruited, participants were given information about the study's purpose and procedure, informed consent was obtained, and interviews were held by the first author between March of 2012 and April of 2013 at a location deemed appropriate by the participant. The interviews lasted approximately 90 minutes and were guided by a structured protocol of open-ended questions with follow-up probes. The interviewprotocol was derived from existing literature on stigmatisation and stigma by association (e.g., Angermeyer et al., 2003; Corrigan et al., 2006), validated scales for assessing experiences of stigma by association and psychological distress (e.g., Mental Health Inventory, Veit \& Ware, 1983; Stigma-by-association scale, Pryor et al., 2012), and 
previously used protocols (e.g., Interview-protocol, Stutterheim et al., 2011). A pilot protocol with themes, questions, and follow-up probes was participatively pretested among family members of people with mental illness, mental health professionals, and people with mental illness, and then adjusted based upon the feedback provided. The revised protocol was then tested again in three interviews with family members of people with mental illness and adjustments were once again made based on the feedback. The final protocol explored:

1. Participants' stigma by association experiences and their perceptions regarding how their community views mental illness and family members of people with mental illness;

2. Participants' perceptions regarding the extent to which they felt that stigma by association experiences have affected their lives and well-being; and

3. Participants' perceptions regarding the extent to which familial relationship, coresidence, and gender are relevant to stigma by association experiences.

All interviews were recorded with a digital voice recorder and transcribed verbatim. Data were processed with QSR NVivo 9.0 and thematic content analysis was conducted (Burnard, Gill, Stewart, Treasure, \& Chadwick, 2008; Thomas, 2006). In this process, the first author first listened to the recordings and then thoroughly examined the transcripts to identify relevant themes and items (open coding; see Table 3.3). Subsequently, themes and items were categorised by the first author (RvdS), with support of second (SS) and fifth author (AB), by identifying similarities and differences in the data. Themes and items were then further explored and a hierarchical data structure based upon these themes and items was developed (Bazeley \& Jackson, 2007; Polit \& Beck, 2010). The creation of this data structure with nodes and attributes was subject to discussion and consultation among all authors and thus evolved into a concerted node structure that accounted for all data. Changes to the hierarchical data structure and the reasons for those changes (i.e., a decision trail) were maintained (Burnard, 2004; Polit \& Beck, 2010).

\section{RESULTS}

Participants reported various experiences of stigma by association. They also spoke of the kind and impact of their familial relationship with a people with mental illness, provided opinions about the role of co-residence, and discussed gender aspects in the context of their experiences of stigma by association (see Table 3.4 for the reported items per participant). 
Table 3.3 Categorised Themes and Items

\begin{tabular}{|c|c|}
\hline Themes & Items \\
\hline Public stigma & Being taken serious/Incompetent \\
\hline Stigma by association & Avoidance \\
\hline Psychological distress & Dangerous \\
\hline Perceived closeness & Negative treatment \\
\hline Family support & Social exclusion \\
\hline Suicide & Find him or her strange \\
\hline Familial relationship & Self-imposed withdrawal \\
\hline Family role & Shame \\
\hline Gender & Guilt \\
\hline \multirow[t]{30}{*}{ Housing } & Concealment advice \\
\hline & Overprotective \\
\hline & Pity \\
\hline & Unpredictable/Untrustworthy \\
\hline & Contagiousness/Heredity \\
\hline & Appreciated/Wanted/Loved \\
\hline & Nervous/Tense \\
\hline & Anxious/Worried \\
\hline & Depressed/Sad/Downhearted \\
\hline & Moody/Brooded \\
\hline & Emotionally unstable \\
\hline & Financial hardship \\
\hline & Loneliness \\
\hline & Exhausting \\
\hline & Stress/Irritation \\
\hline & Time consuming \\
\hline & Justice/Prosecution/Criminal activities \\
\hline & Quarrel/Tussle \\
\hline & Missed career opportunities \\
\hline & Less close and caring family \\
\hline & Contact \\
\hline & Domestic support \\
\hline & Financial support \\
\hline & Familial relationship and family role \\
\hline & Caregiver respite \\
\hline & Gender differences \\
\hline & Impact living together \\
\hline & Being yourself \\
\hline & Having your own friends and company \\
\hline & Rooms/Studying \\
\hline
\end{tabular}




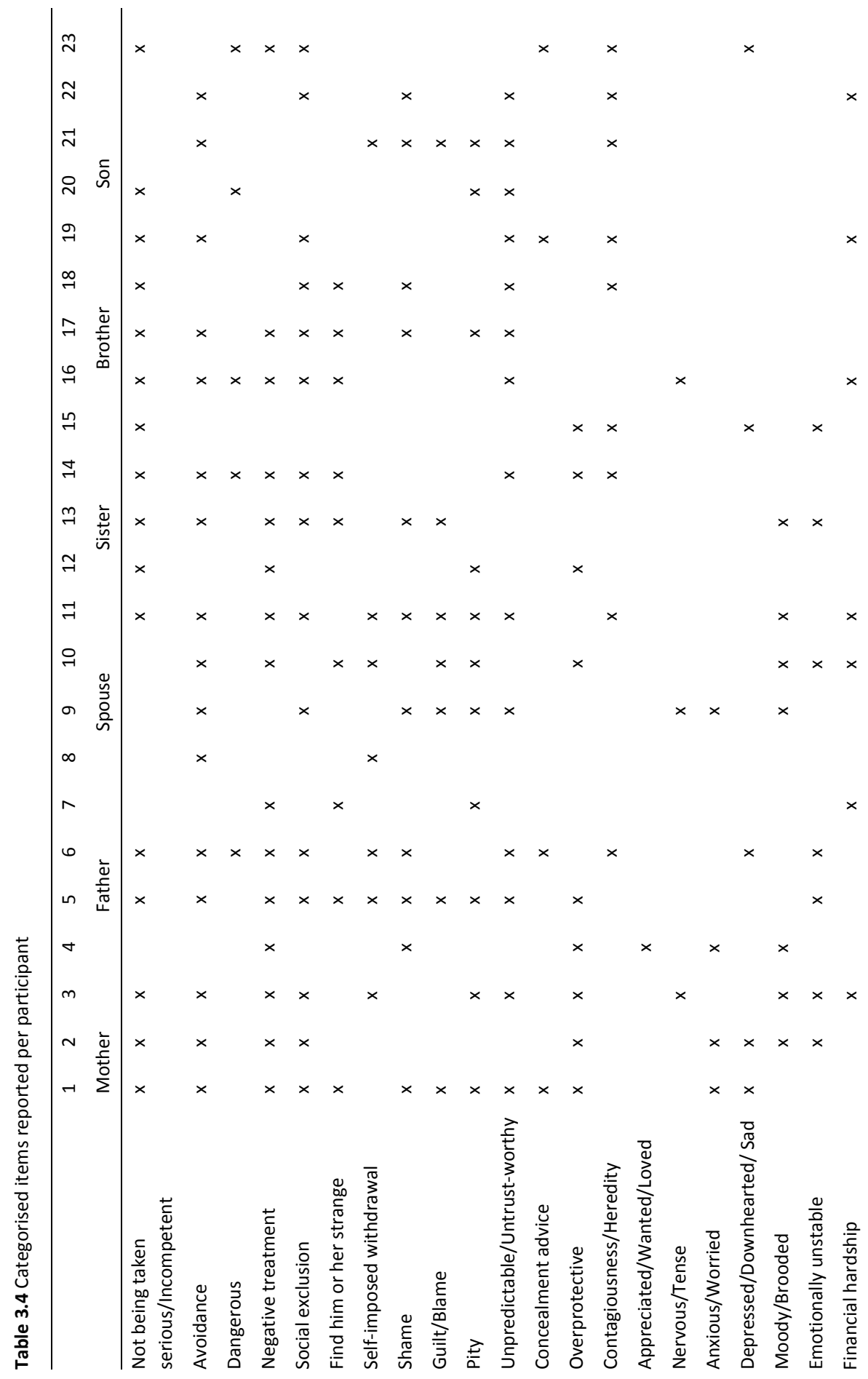




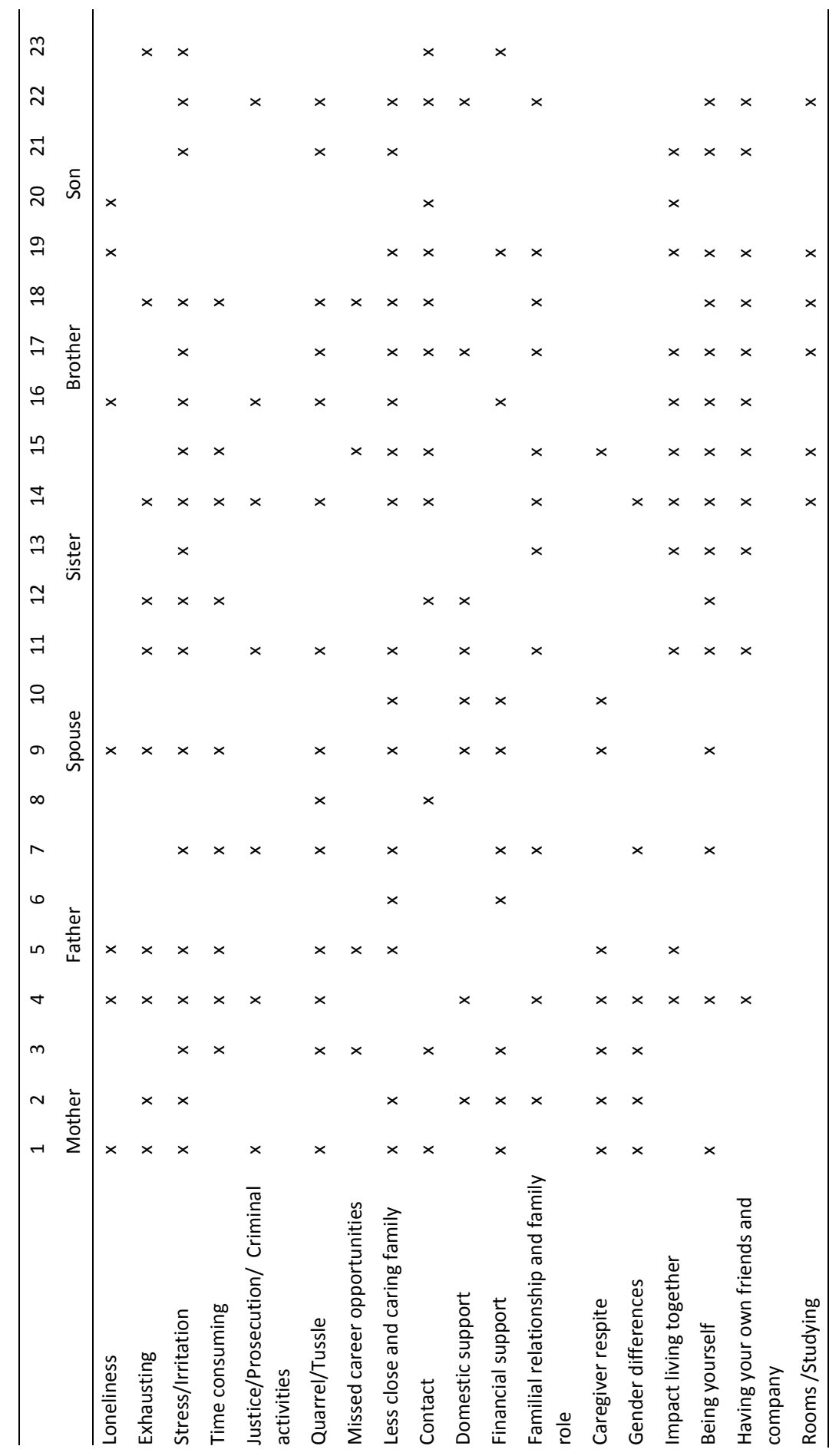




\section{Experiences of stigma by association}

Participants claimed that not only are people with mental illness affected by stigma, they, as family members, are affected as well. They indicated that their community members often have oversimplified images of mental illnesses, of people with mental illness, and of their family members. They also reported community members having negative attitudes and displaying discriminatory behaviour towards people with mental illness and their family members:

I was getting ready to go for a walk and I was outside. I put him [her youngest son] in the stroller and I was locking the door and stuff. So, this little boy [neighbour] comes over to my son [without mental illness] and wants to talk to him and what does my neighbour do? She pulls that kid away from him [participant's son] and hollers at me, 'That's contagious!' I think, 'Contagious? What are you talking about?' 'Yeah, contagious what you guys have and he [participant's - absent - son with mental illness] even takes that bus to that special school, so it's contagious.' (Karen, mother, age 58)

Participants mentioned experiencing stigma by association regularly, and found themselves "labelled" as incompetent, responsible for the stigmatised condition, unpredictable, and different:

We were also considered pitiful, weird, and unpredictable, and they didn't take us seriously when we asked them to help. It was our own fault and things would just work themselves out. (Celia, sister, age 46).

Participants further reported experiencing social distance, having fewer social contacts, and smaller social networks because they had a family member with mental illness. They spoke of avoidance, rejection, and abandonment:

Speaking of stigmatisation, those two girls, they'd been really good friends for at least five years and then, out of the blue, contact was discontinued because the father was psychotic. The daughter was looked down upon. She was no longer welcome there and contact had to be broken because her father had had a psychotic break. (Karen, mother, age 58)

In fact, almost all participants described changed or broken relationships with family, being alienated from friends, or having smaller social networks as a result of stigma by association and manifest as social distance, blame, or negative remarks and dismissal: 
Your world becomes smaller and smaller. You have fewer social contacts, you lose friends, and you become socially isolated. It just seems to happen over time. (Roy, husband, age 45)

In addition to experiencing altered or broken relationships and being alienated from friends and extended family members, participants also spoke of being inhibited in social situations from speaking openly about their family member:

You know, when you're with company, they say, ahead of time, 'Let's keep things light. Let's not talk about that stuff, your sick daughter or the difficulties or how hard it is for you'. People want you to be the old you. Everything's fine. Everything should be fine. Don't talk about it. Don't be weird. Some even avoid you for a good long time. (Suzanne, mother, age 59).

Some participants indicated that they reduced contact with family members, friends, and acquaintances, and avoid some forms of social engagement in order to avoid having to talk or not talk about their family member with mental illness. Participants also mentioned avoiding social events because they did not wanted to be confronted with stigmatising reactions by others.

\section{Stigma by association in contact with mental health professionals and civil servants}

Although not explicitly investigated by our interview-protocol, a theme that frequently arose in the data was stigma by association in contacts with mental health professionals and civil servants. In fact, more than half of the participants in this study explicitly mentioned having experienced stigma by association in these contacts. Parents and spouses, in particular, experienced being held responsible and blamed by civil servants and mental health professionals for their family members' mental illness:

And then the first one came, ready with his bias. He was from children's services. That man, he came inside and we were shocked. He showed up and was like,... 'Parents, you've done it all wrong.' He had an enormous preconceived idea. He stigmatised us. We needed more than half an hour to convince him that we had done everything we could and that we had two other children and there were no problems with them. You constantly need to defend yourself. (Renske, mother, age 59)

Additionally, participants reported perceiving a lack of interest on the part of civil servants and mental health professionals. They often felt that their perspectives on care, their familial relationship with their family member, and the problems and concerns present were not taken seriously: 
Being taken seriously? In healthcare? I've become so frustrated [hits table]. As the mother of a sick kid, mothers don't count, mothers are no longer part of it. They [children with mental illness] are no longer your problem [points to forehead to indicate 'crazy']. Of course they are, but then, all of the sudden, it's your fault. You're part of the problem. (Emma, mother, age 59)

In fact, many participants reported feeling ignored by professionals and complained that they had been excluded from the treatment process - that professionals had rejected their cooperation and involvement despite having been, often for years, the sole provider[s] of support to their family member[s] with mental illness. They further reported feeling as though they were seen as incompetent, a nuisance, and an additional burden. Several parents and spouses reported feeling particularly frustrated and angry because they had, for a long time, been fully responsible for their family member, had backed and supported their child or spouse for years, and then, when professional treatment or hospitalisation occurred, they felt they were 'left out of the team' and did not get adequate information.

And then, when he was admitted - he went there himself - and I wanted to know how he was doing. I was put out in the hallway where everyone can walk by and there I was told that they wouldn't give me any information because he didn't want that. I wasn't even invited into the office. Nothing. People were there walking around, visiting and doing whatever and they could hear everything. That's mental health services for you. But you're so tired, so incredibly tired, dog-tired. You're no longer resilient or you're so resilient that you get mad. I, at that point in time, you might say, 'flipped out'. Nope, nothing. No, I didn't feel taken seriously at all. In fact, I felt like they looked down on me. I felt like they had pushed me back into a corner. (Anja, wife, age 61)

\section{Familial relationship, co-residence, and gender}

In their interviews, participants discussed the extent to which they perceived their familial relationship, co-residence, and gender to be relevant to their experiences of stigma by association.

\section{Familial relationship.}

Participants that were differently connected to their family member with mental illness did appear to report different kinds of stigma by association experiences. Parents and spouses regularly reported having been held responsible for the onset and perpetuation of their family member's mental illness, as exemplified by comments about them being a poor or less competent parent or spouse: 
I felt like she held me responsible. There were remarks about me not being a good mother. I totally panicked and I drove home and I thought that I had indeed done it all wrong. (Emma, mother, age 59)

They told me, 'Do something about it!' But what could I do? Go and hide all the money and food in the house? And they certainly blamed me for her condition. What could I do? Force her to adhere to treatment? (Dick, husband, age 54)

In contrast, children and siblings spoke more generally about experiencing stigmatisation, mostly from other parents and children in their communities and mainly in their puberty and adolescence. Most frequently reported among siblings and children was been seen as different or even deviant by others. This subsequently evoked shame and often led to efforts to conceal the relationship with a person with mental illness in order to avoid negative reactions and the potential loss of friendships and even (potential) romantic relationships:

Then I thought, 'Fuck off! Get out of my life!' In those moments, I felt ashamed and I always considered it [when making plans with friends] and avoided her and those situations and the contact. I never talked about her. (Joep, brother, age 43)

Children and siblings of people with mental illness reported that, although experiences of stigma by association were particularly stressful during their puberty and adolescence, as they grew older and moved out of the family home, stigma by association experiences lessened and became more manageable.

Another crucial time for children of people with mental illness occurred when they started a family of their own. One participant mentioned how family members and acquaintances advised her not to have children because of the mental illness in her family. Similarly, some children and siblings of people with mental illness reported being concerned that their children would inherit their family member's mental illness, would later on experience stigmatisation, and that history [mental illness stigma and stigma by association] would repeat itself in yet another generation:

But, you do it anyhow [have children]. I mean it's not only hard for you as a parent but also for that kid. I think it would be horrible [to have schizophrenia]. So yes, I've considered it when thinking about whether I want to have children. (Liza, sister, age 30). 


\section{Co-residence}

One participant, who had previously lived together with his sister with mental illness, mentioned taking her mental illness, and even her physical presence, into account when with friends, especially when he invited friends over to his house. He felt her behaviour brought stigmatisation upon both her and him and that he too would be perceived as different or deviant. He also felt that he had had fewer opportunities to meet and connect with others because of his sister's mental illness, her behaviour, and their co-residence:

You become calculated in your interactions and you take her presence, or rather her absence, into account when you plan something - who, what, when, with what. You avoid her and her illness and you don't tell anyone but because we, soon after, no longer lived in the same house, it was liveable and I was less ashamed than I would have been had I still lived in the same home. (Joep, brother, age 43).

This kind of experience was quite common among siblings and children who lived together with their family member with mental illness. Often, participants conveyed how their perceived experiences of stigma by association and resulting distress increased when they, mostly in their early teens, became increasing aware of others' reactions to their family member with mental illness and the family as a whole. These participants reported that they, especially in their puberty and adolescence, actively avoided their family member in order to prevent or mitigate experiences of stigma by association. They also mentioned that their experiences of stigma by association decreased substantially when they left the household where they and their family member lived together:

I moved in with my boyfriend sooner because of it [sister's mental illness]. I left. I left the situation at home. She lived there, my parents lived there, and I didn't want to be seen as part of the problem anymore. I left and so I left a lot of it behind. When you move out, you're no longer right in the middle of it and you're no longer part of it and I didn't want to be part of it anymore. (Ria, sister, age 45)

\section{Gender}

The data suggests that women are more frequently held responsible for the mental illness of a family member than men are. In fact, many of the women in this study reported experiencing or observing accusations that they were or had been overprotective and thus had caused, contributed, or sustained their family members' mental illness:

My in-laws found me to be overprotective. I kept the problem going on they said. They told me to throw my son out of the house and to let him be. Accord- 
ing to them, I was overprotective and I sustained his mental illness and his problems. (Natasja, mother, age 57)

Another participant recalled her mother being accused several times of being overprotective and as such causing and continuing her daughter's mental illness:

That happened a lot. It happened to my father and my mother too. They were told all sorts of things but it was mostly my mother and it was mostly about her being too protective. She needed to be firmer, that it was her fault because she wasn't firm enough with her [daughter with mental illness]. (Celia, sister, age 46)

Interestingly, these accusations appeared to be geared exclusively to female family members of people with mental illness, and particularly mothers, as none of the male participants in our study reported such accusations.

\section{DISCUSSION}

This study set out to document the experiences of stigma by association among immediate family members of people with mental illness, while taking into account relevant influencing factors including familial relationship, co-residence, and gender.

\section{Stigma by association}

Participants reported a broad range of stigma by association experiences. The findings suggest that family members of people with mental illness experienced negative treatment, not being taken seriously, but above all they experienced and observed other immediate family members being blamed and being held responsible for their family members' mental illnesses and behaviour. They also reported that stigma by association disrupted social relationships and led to social distance. Family members indicated that these experiences were very stressful and diminished their well-being. This is in line with Angermeyer et al. (2003) and Van der Sanden et al. (2013) who found that stigma by association of families of people with mental illness leads to psychological distress, diminishes well-being, and negatively impacts their relationships with others. Previous research has shown that members of the general public often manifest an immediate and sometimes even unconscious aversion to people with a stigmatised condition that can be followed by discriminating and devaluating behaviour (Stutterheim et al., 2011). Weiner et al. (1988) have shown that stigmas that were perceived as more controllable elicit less pity, arouse more anger, and lead to accusations of negligence. Most likely, the onset and continuation of mental illnesses of people with men- 
tal illness are perceived by the general public as, at least partly, controllable by the family members of people with mental illness (Weiner et al, 1988). For this reason, family members appear to be seen as personally responsible for their family member's mental illness and the stigmatised condition, leading to experiences of stigma by association.

Yet, in regard to the experiences of stigma by association upon family members of people with mental illness in our study, social distance appeared to not only derive from the actions of others; self-imposed withdrawal from existing social networks and social events occurred as well. Family members of people with mental illness appeared to make specific choices with regard to which social networks and social events they wanted to maintain. It is possible that these participants were sensitive to negative experiences, including stigma by association, and thus avoided social experiences and social events to reduce the risk of rejection and stress (Philips \& Benoit, 2013). Withdrawal from one's social network and social events might also have been necessary to prevent emotional exhaustion (Verhaeghe \& Bracke, 2012).

\section{Stigma by association in contacts with mental health professionals and civil servants}

More than half of the participants in this study reported experiencing stigma by association in their contacts with mental health professionals and civil servants. Participants also reported feeling little to no support in handling the burden of being related to a person with mental illness from mental health professionals or civil servants. These findings are in accordance with Östman and Kjellin (2002) who found family members of people with mental illness to have stigmatising experiences with staff members of mental health hospitals.

While mental health professionals and civil servants are likely well trained in the individual rights of and their legal obligations towards people with mental illness, they may not give sufficient consideration to family members' familial responsibilities, adjoining problems, and their justifiable concerns, and this is likely to be what is perceived as stigma by association by family members. Addressing this is important as perceived exclusion from the treatment processes may lead to diminished social support provision to people with mental illness by family members during periods of hospitalisation and thereafter. Also, a lack of adequate information provision due to privacy regulations or medical confidentiality can be perceived as very stigmatising by family members. This is consistent with Angermeyer et al. (2003), who found inadequate flows of information to be regarded as a form of structural discrimination. However, Angermeyer et al. (2003) also noted that family members' critique that they had been blamed by mental health professionals and civil servants may not necessarily imply that they were 
explicitly blamed but rather family members of people with mental illness may react sensitively to possible accusations or signs of rejection.

\section{Familial relationship}

In our study, experiences of stigma by association appeared to be impacted by the familial relationships participants had with their family members with mental illness. Parents and spouses seemed to have rather similar experiences of stigma by association (i.e., avoidance, being blamed, and negative treatment) and, as a consequence, they felt ashamed, guilty and sometimes helpless, whereas siblings and children mainly reported rejection and abandonment, and feared contamination. These findings are partly similar to those of Corrigan and Miller (2004) who found that parents tend to be blamed for the onset of their children's mental illnesses, spouses and siblings tend to be held responsible for not assuring their family members adhere to treatment plans, and children tend to be fearful about becoming contaminated by their parent's mental illness. The similarity between parents and spouses in their experiences of stigma by association may emerge from the general publics' view on parents' and spouses' societal roles as caregivers, and their perceived personal responsibility for the familial relationship and stigmatised condition (Bos et al., 2008; Corrigan \& Miller, 2004; Weiner et al., 1988). In this context, parents and spouses face blame because they are held responsible for the upbringing of their children or for influencing and correcting their spouses (Angermeyer et al., 2003). Children and siblings of people with mental illness in this study, however, were more infrequently deemed the role of caregiver, and were thus less likely to be considered personally responsible for the mental health condition of their family member, moreover as they did not voluntarily choose the familial relationship. Furthermore, none of the siblings in this study reported the family member with mental illness to obstruct or not to adhere to treatment plans. Possibly, for these reasons did siblings in this study not report experiences of being held responsible for treatment nonadherence. Nevertheless, siblings and children appeared to be seen as potentially deviant people as a result of their association with a person with mental illness, especially by their peers during their puberty and adolescence, resulting in abandonment and social rejection. Clearly, the different kinds of familial relationships appear to have different experiences because they have different roles and responsibilities within the family (Eldering, 2006).

\section{Co-residence}

The results in this study further suggest that co-residing with a person with mental illness may impact stigma by association experiences. Family members, especially siblings, reported that living together with their family member with mental illness increased their experiences of stigma by association and impacted the frequency and 
context of their social contact with others. These findings are in line with Conde-Sala, et al. (2010), who found in their study among caregiving family members, that living with a person with mental illness had a notable influence on the social burden, and psychological distress among these family members, and as such worsened their mental health condition. Lanquetot (1984), in her report on being an immediate family member of people with mental illness found that co-residence reinforced feelings of shame, embarrassment, social exclusion, rejection by friends and acquaintances, concealment, and the need of getting away from home and to escape from the stigmatised situation. It is quite possible that living together with a family member with mental illness makes the familial connection more noticeable and salient, decreases the success of efforts to conceal or selectively disclose one's connection to a person with mental illness, and thus lay the groundwork for increased stigma by association (Dovidio et al., 2000; Lanquetot, 1984; Kulik et al., 2008). In that way, living with a family member with mental illness may exacerbate experiences of stigma by association as social exclusion, rejection, and abandonment, and increase one's psychological distress (Bos et al., 2009).

\section{Gender}

In contrast to male participants, female participants frequently reported others thinking they are overprotective and, through that overprotectiveness, they perpetuate, maintain, or sustain their family members' mental illness. These findings are analogue to findings by Wirth and Bodenhausen (2009), who found that gender typical cases of mental illness, symptoms, or behaviour elicit negative affect, less sympathy, and less support. Possibly, women are seen as more caring than men and are thus seen as overprotective or patronising by others. This appears to be consistent with the social role theory proposed by Eagly, Wood, and Diekman (2000), a theory that contends that men and women behave differently in social situations and take on different roles as a result of different expectations placed upon them by society. As such, overprotectiveness may be perceived as a gender-typical behaviour by the general public. Gender differences, gender-typical symptoms, and stigmatisation has been previously studied by Wirth and Bodenhausen (2009) and, although their study focused on the relationship between gender and public stigma, some of the processes they uncovered may analogue to the processes at play in the relationship between gender and stigma by association. Specifically, Wirth and Bodenhausen (2009) examined whether a person with mental illness' gender moderates the degree of stigma experienced and found that gender-typical behaviour is attributed to the personal traits of the actor, what may result in greater blame, and being held personally responsible for the stigmatised condition (Weiner, 1995; Wirth \& Bodenhausen, 2009). In the context of female family members of people with mental illness, it may be that overprotectiveness lends itself to blaming as it is perceived by others to represent typically female behaviour and may 
lead them to hold these women personally responsible for the onset, or continuation of their family members' mental illness (Wirth \& Bodenhausen, 2009).

\section{Strengths and limitations}

Our study has both strengths and limitations. The primary strength is the inductive nature of our research and the extensive and 'thick' descriptions that capture the contextual experiences of stigma by association among family members of people with mental illness. A second strength is the involvement of people with mental illness, their family members, and mental health professionals, as well as the application of previously validated scales and protocols, in the development of this study's interview protocol. We set out to ensure the quality, reliability, and validity of this qualitative study by using this semi-structured interview protocol to guide the interviews. Furthermore, rigour was sought by maintaining a transparent decision trail throughout the study, by checking our findings with relevant stakeholders, and through theory triangulation (Polit \& Beck, 2010).

Our study also has some limitations. Given that our sample size was, although adequate for an exploratory study, small, caution should be applied in generalising the results from this study to other populations. At the same time, it is important to recognize that qualitative research does not necessarily seek to attain generalizability. It can, however, be transferable and thus invites readers to make connections between elements of this study and their own experiences (Lincoln \& Guba, 1985). Another limitation was that study participants were mainly recruited through support groups. Recruitment through support groups may have led to selection bias as those participants who seek support may be more unsatisfied with professional services, or because those who volunteered may be the most dissatisfied among group members. On the other hand, the interviews, and interview-protocol principally focussed on experiences of stigma by association in a settings beyond mental health services, namely in participants' communities and society as a whole. Another potential limitation was the use of retrospective reports and subsequent recall bias. Recall bias was, however, combatted, at least to some extent, by asking follow-up questions to attain clearer and more detailed insight regarding participants' experiences of stigma by association. Lastly, in our study, coding was conducted primarily by the first author. Having multiple coders and determining interrater reliability may have enhanced the rigour of this study.

\section{Practical implications}

Our findings suggest several practical implications. First, they suggest that there is a need for the provision of tailored support to family members of people with mental illness that takes familial relationship, co-residence, and gender into account. Specific 
attention should be paid to the particular needs of immediate family members who frequently act as caregivers, and siblings and children of people with mental illness in their puberty or adolescence. Services and facilities that provide respite from caring for a person with mental illness are likely to be highly beneficial. Furthermore, the extent to which family members provide social support and help their family member should be acknowledged and reinforced by community members, and mental health professionals. It is also important that mental health professionals should be aware of the stigmatising effect and stress that can result from inadvertently excluding immediate family members from treatment processes, and inadequate flows of information. We therefore recommend that in-service training for mental health professionals and civil servants include the provision of information, create opportunities for discussion, and build skills as they pertain to how professionals can best support family members and acknowledge their responsibilities and concerns.

\section{Conclusion}

In conclusion, this study has contributed to our understanding of various processes underlying stigma by association among immediate family members of people with mental illness. Participants reported that stigma by association experiences has negatively impacted their psychological well-being and social lives. Also, this study has shown that familial relationships, co-residence, and gender affect the stigma by association experiences of family members of people with mental illness. The relevance of these characteristics combined with the variety of stigma by association experiences reported point to the need for tailored education and emotional support provision to family members. Furthermore, the findings indicate that mental health professionals and civil servants need to acknowledge family members' familial relationships and responsibilities, in particular, the support they have provided to their family member with mental illness and their fears, concerns, and problems in that context. In-service training for mental health professionals and civil servants should, therefore, include opportunities for debate, discussion, and social skills development as they pertain to these needs.

We recommend that future research include longitudinal research that involves people with mental illness and their immediate family members and that explores the interactions between perceived stigma by association, self-stigma, and self-withdrawal. Further quantitative research on the possible relationship between the type of mental illness and stigma by association experiences is recommended as well. Additionally, future research should take familial relationship, co-residence, and gender into account. 



\section{CHAPTER 4}

\section{COPING WITH STIGMA BY ASSOCIATION AND FAMILY BURDEN AMONG FAMILY MEMBERS OF PEOPLE WITH MENTAL ILLNESS}

This chapter has been published as:

Van der Sanden, R. L. M., Stutterheim, S. E., Pryor, J. B., Kok, G., \& Bos, A. E. R. (2014). Coping with stigma by association and family burden among family members of people with mental illness, Journal of Nervous and Mental Disease, 202(10), 710-717. doi:10.1097/NMD.0000000000000189 


\section{ABSTRACT}

In this study we explored stigma by association, family burden, and their impact on the family members of people with mental illness. We also studied the ways in which family members coped with these phenomena. We conducted semi-structured interviews with 23 immediate family members of people with mental illness. Participants reported various experiences of stigma by association and family burden. Social exclusion, being blamed, not being taken seriously, time-consuming caregiving activities, and exhaustion appeared to be the predominant forms of stigma by association and family burden experienced by participants. The participants used problem-focused and emotionfocused coping strategies, separately or simultaneously, to cope with the negative impact of stigma by association and family burden. The results suggest family members should have access to services to address these problems. Social, instrumental and emotional support should be given to family members by community members and mental health professionals.

Keywords: stigma by association, stigma, mental illness, burden, coping 
People with mental illness are confronted with stigma regularly. They have been discriminated against in several ways throughout the centuries, and public images of people with mental illness still tend to be very negative (González-Torres., Oraa, Arístegui, Fernández-Rivas, \& Guimon, 2007). People with mental illness are often seen as being deviant, dangerous, and less competent than others (Wahl, 1995). The stigma associated with mental illness is known to have a tremendous impact on the daily life and the life satisfaction of people with mental illness; it also contributes to unemployment and low income among them (Markowitz, 1998). Furthermore, people with mental illness often experience a reduction in social contact and an increase in social rejection, which may lead to social isolation and diminished self-esteem (Bos et al., 2009).

Most family members of people with mental illness who have participated in relevant surveys and research in the Netherlands have contact with their family member with mental illness at least once a week. They have regular contact in person, by telephone, or by e-mail. Some of them see each other daily, and many are involved in caregiving activities for their family member with mental illness (Van Erp, Place, \& Michon, 2009). They usually support their family members practically, helping with housing, food, and money as well as giving emotional support. Family members of people with mental illness in the Netherlands reported positive and negative effects of their family member's mental illness (Kwekkeboom, 2000). They learned to accept their family member's deviant behaviour and felt appreciated for their caregiving activities, yet $75 \%$ reported regularly being irritated by his/her behaviour, tensions within the family, or disruption to their life because of their family relationship with a person with mental illness (Kwekkeboom, 2000).

It has been shown that the stigma of mental illness affects not only people with mental illness but also their family members by the mere association to them. This phenomenon is called stigma by association (Neuberg et al., 1994). Family members of people with mental illness who had experienced stigma by association reported stress and anxiety, a reduction in social interactions, social exclusion, and negative treatment. As a result of these experiences, some of them devoted energy and resources to concealing their relationship to the family member with mental illness (Larson \& Corrigan, 2008; Van der Sanden et al., 2013). The behaviour of a person with mental illness may further isolate his/her family, diminish their reputation, and jeopardise their relationships with friends and neighbours (González-Torres et al., 2007).

Family members of a person with mental illness also have to deal with family burden (Farina et al., 1992; Judge, 1994). Platt (1985) presented one of the first definitions of burden; this definition referred to difficulties, problems, and adverse events affecting people with mental illness and their families (Schene, 1990). Although the term burden is sometimes perceived as a stigmatising term, its use in research to refer to "all the 
difficulties and challenges experienced by families as a consequence of someone's illness" (Ennis \& Bunting, 2013, p. 255) is well known, and it is in this sense that it is used in this study. Several researchers argued that family burden was a multidimensional concept because the problems and challenges that may be experienced by family members of someone with a mental illness include financial hardships, time-consuming caregiving activities, disruption of normal household routines and roles, as well as psychological distress related to the illness of this family member (Schene, 1990). Family burden is frequently categorised descriptively as objective burden or subjective burden. Objective burden includes the factual negative impact and problems experienced by the family of a person with mental illness as a result of the mental illness. Subjective burden includes the negative emotional impact on the family of a person with mental illness and the psychological distress caused by his/her mental illness (Tsang et al., 2003).

Several researchers have classed stigmatisation as a dimension of subjective burden or described stigmatisation as a psychosocial burden (De Boer et al., 2008; Sales, 2003; Schene et al., 1996). Werner et al. (2012) even reported that stigma by association was a significant predictor of family burden, "above and beyond the influence of more traditional predictors" (p. 91 ). Nevertheless, there are other definitions and indices of family burden that do not refer to stigmatisation or stigma by association. We therefore suggest that although stigma by association may be related to family burden and their consequences may overlap to a certain extent, the two constructs are not identical. Most importantly, the overlap between the impact of stigma by association and family burden seems to depend on the definitions and operationalisation of the concepts.

Nonetheless, experiences of being stigmatised and the burden of a family member's mental illness do threaten the physical, psychological, emotional, and functional health of the family of a person with mental illness (Angermeyer et al., 2003; Phelan et al., 1998). Experiences of being stigmatised affect their levels of self-esteem, stress and anxiety, and their social performance (Rüsch et al., 2009); these are important issues in psychiatric rehabilitation because they affect both the recovery of the person with mental illness and his/her family.

Stigma by association and burden are major stressors for the family of a person with mental illness and may lead to use of a variety of coping strategies to mitigate their negative impact (Carver, Scheier, \& Weintraub, 1989; Miller \& Kaiser, 2001). Coping strategies are often categorised into problem-focused coping strategies (i.e., use of instrumental support, use of emotional support, active coping, planning, and use of humour) and emotion-focused coping strategies (i.e., venting, self-blame, acceptance, substance use, behavioural disengagement, denial, self-distraction, positive reframing, and religion) (Carver et al, 1989; Eaton et al., 2011; Stutterheim, 2011). Problem- 
focused coping strategies are intended to reduce or eliminate the negative impact of a stressor and facilitate recovery of function, whereas emotion-focused coping strategies are focused on managing the negative emotions associated with the stressor (Carver, 1997; Eaton et al., 2011; Tuncay et al., 2008). Lazarus and Folkman (1984) found that participants' appraisal of a stressor strongly predicted the coping strategies they would use. If the stressor was perceived to be relatively manageable or controllable it was more likely that participants would use problem-focused coping strategies; if they perceived the stressor to be relatively unmanageable or uncontrollable, they were more likely to choose emotion-focused coping strategies.

However, stigma by association, family burden, and the ways family members of people with mental illness cope with them have not yet been studied extensively. In this qualitative study, the experiences of stigma by association and the burden on the immediate family of people with mental illness were investigated in more detail. We also explored how family members coped with stigmatisation and burden because this may improve our understanding of how experiences of stigma by association and family burden affect the family of a person with mental illness and suggest possible interventions aimed at reducing their negative impact.

\section{METHOD}

\section{Participants and Procedure}

This qualitative study explored experiences of stigma by association and burden among members of the immediate family (i.e., parent, child, sibling, or spouse) of people with mental illness. The Research Ethics Board of the School of Psychology and Neuroscience at Maastricht University approved the study.

Between March 2012 and April 2013, a total of 23 members of the immediate family of a person with mental illness participated in face-to-face, semi-structured interviews (another manuscript, exploring the relationships between sex, family role, co-residence and stigmatisation was submitted on the basis of a separate part of the dataset [Van der Sanden, Bos, Stutterheim, Pryor, \& Kok, 2014]). All participants were Dutch Caucasian men (11) or women (12). Their ages ranged from 25 to 64 years, with a mean age of $44.3(S D=12.6)$ years. The participants' characteristics are shown in Table 4.1. The types of mental illness reported varied as follows: depressive, bipolar or other mood disorder, 12 persons; personality disorder: 4; attention deficit hyperactivity disorder or attention deficit disorder: 4; dissociative disorder: 3; autism: 3; schizophrenia or other psychotic disorder: 2; addiction: 1 . At the time of interview, 12 participants were in contact with the family member with mental illness on a daily or weekly basis, 8 did not 
have regular contact with that family member (i.e., contact approximately four or fewer times a year), and in 3 cases the family member with mental illness was deceased.

Table 4.1 Demographic and Background Characteristics of Sample $(N=23)$

\begin{tabular}{ll}
\hline Variable & Percentage (\%) \\
\hline Sex & 47.8 \\
Male & 52.2 \\
Female & \\
Age & $44.3(12.6)$ \\
Years (mean (SD)) & $25-64$ \\
Range (min-max) & \\
Marital status & 13.1 \\
Single & 52.1 \\
Married & 21.7 \\
Divorced & 13.1 \\
Widowed & \\
Family relationship & 20.7 \\
Spouse & 20.7 \\
Child & 27.5 \\
Parent & 31.1 \\
Sibling & \\
\hline
\end{tabular}

The participants were recruited through announcements distributed by Dutch mental health institutes and mental health self-help groups (e.g., Labyrint-in-Perspectief, Vereniging Geestdrift, Zorgbelang Brabant, Stichting Borderline, Stichting Caleidoscoop); four participants were recruited directly by the first author. After recruitment, the participants were given further information about the purpose and procedures of the study. Informed consent was obtained from all participants prior to interview. All participants were interviewed for approximately 1.5 hours at a location deemed appropriate by participant. Interviews continued until theme saturation was achieved. To improve the rigour of the research, all interviews were recorded with a Dictaphone and transcribed verbatim.

The interviews themselves were guided by a structured protocol consisting of openended questions and follow-up probes. The protocol explored participants' experiences of stigma by association and burden, including the extent to which stigma by association and burden affected the participant's life and well-being as well as the ways in which the participant coped with these experiences. The questions asked of the participants in this study were divided among the introductory part of the interview and six main themes: 
1. their family, the mental illness of the relevant family member, and their relationship with this family member

2. their experiences of stigmatisation of people with mental illness

3. their experiences of stigmatisation of the family of people with mental illness

4. burdens they experienced as a family member of a person with mental illness

5. their coping strategies and the perceived effectiveness of these strategies

6. further themes, items, and questions participants thought were relevant to our research

The questions and follow-up probes in a pilot protocol were developed from current literature, previously used protocols, and validated scales for assessing the experiences and consequences of stigma by association, burden, and coping (e.g., Mental Health Inventory, Veit \& Ware, 1983; brief COPE scale, Carver, 1997; Stigma-by-association scale, Pryor et al., 2012; Burden scale, Pryor et al., 2012; Interview protocol, Stutterheim, 2011). The questions and themes in this pilot version of the protocol were presented to several professionals, peers, people who had a family member with mental illness, and people with mental illness, and adjustments were made to the protocol and topic guide based on their input. Next, the protocol - questions and follow-up probes - was tested in pilot interviews with three persons who had a family member with mental illness. Their remarks and input prompted further adjustments to the protocol. During these pilot interviews, participants were explicitly invited to give their opinion of the research questions and asked whether the interview had missed essential questions or themes; thus, our protocol evolved and was enriched through partnership with the participants.

To manage and analyse the data, the interview transcriptions were imported into a computer software package for qualitative data analysis (QSR NVivo 9.0). Data were processed and analysed using a general inductive approach and thematic content analysis (Burnard et al., 2008; Thomas, 2006). The first author undertook "open coding" and examined recordings and transcripts thoroughly to identify relevant subjects. Subsequently, the researchers started describing, analysing, organising, and categorising variations on themes and concepts by identifying similarities and differences in the data. Themes and concepts were then further investigated and a node structure (i.e., hierarchical data structure) based on these themes and concepts was developed (Bazeley \& Jackson, 2007; Polit \& Beck, 2010). The creation of the hierarchical data structure with nodes, child nodes, leaf nodes, and attributes was subject to regular discussion, consultation, and review among the researchers and evolved into a concerted node structure. This node structure accounted for all our data. Changes to the hierarchical data structure and the reasons for such changes were logged. The decision trail was recorded in the same log (Burnard, 2004; Polit \& Beck, 2010). 


\section{RESULTS}

\section{Experiences of stigma by association}

The themes encompassing topics and dimensions that emerged prominently in the interviews in this qualitative study are presented in Table 4.2.

Table 4.2 Themes Emerging From Interviews

\begin{tabular}{|c|c|c|}
\hline Topics prominent in interview & $\begin{array}{l}\text { Dimension } \\
\text { involved } \\
n\end{array}$ & $\begin{array}{l}\text { Frequency } \\
\text { in sample }(N=23) \\
\%\end{array}$ \\
\hline Stigma by association & 17 & 74 \\
\hline Not being taken seriously & 9 & 40 \\
\hline Being avoided & 8 & 35 \\
\hline Negative treatment & te22 & 22 \\
\hline Social exclusion & 6 & 26 \\
\hline Perceived as strange & 5 & 22 \\
\hline Blamed /made to feel guilty & 8 & 35 \\
\hline An object of pity & 2 & 8 \\
\hline Accused of overprotectiveness & 8 & 35 \\
\hline Burden & 20 & 87 \\
\hline Financial & 7 & 30 \\
\hline Loneliness & 8 & 35 \\
\hline Exhaustion & 7 & 30 \\
\hline Stress and irritation & 17 & 74 \\
\hline Time-consuming & 9 & 40 \\
\hline Divorce & 6 & 26 \\
\hline Had to move & 6 & 26 \\
\hline Missed career opportunities & 5 & 22 \\
\hline Family relationships are less close and caring & 9 & 40 \\
\hline Legal action & 7 & 30 \\
\hline Quarrels and tussles & 12 & 52 \\
\hline Coping & 23 & 100 \\
\hline Sharing & 6 & 26 \\
\hline Therapy & 13 & 56 \\
\hline Advocacy and support groups & 17 & 74 \\
\hline Support & 5 & 22 \\
\hline Self-control & 6 & 26 \\
\hline Concealment & 1 & 4 \\
\hline Plan or structure & 8 & 35 \\
\hline
\end{tabular}




\begin{tabular}{lll}
\hline Topics prominent in interview & $\begin{array}{l}\text { Dimension } \\
\text { involved }\end{array}$ & $\begin{array}{l}\text { Frequency } \\
\text { in sample }(N=23) \\
\end{array}$ \\
\hline Withdrawal & 15 & $\%$ \\
Distraction & 7 & 65 \\
Acceptance & 6 & 30 \\
Abandonment & 4 & 26 \\
Conflict & 5 & 17 \\
\hline
\end{tabular}

Seventy-four percent of the participants in this qualitative study reported experiences of stigma by association. They described how these stigmatising reactions affected their mental well-being, social life, and social networks: "They [other community members] tried really hard to find something. It felt like there had to be something to find, there had to be something wrong with you" (Jennifer, sister, age 45 years).

An issue frequently mentioned by participants was changes in family relationships and friendships over time; sometimes, the relationship or friendship even broke up:

Actually I get on well with my nieces and nephews, with my family, but there are some things which the family won't mention to anyone, not even to aunts and uncles. Mental illness wasn't acceptable. No, it didn't fit the image, oh, no! In any case, it's a taboo, and creates distance between us (Carmen, mother, age 58 years).

Several participants reported avoiding social events and reducing or breaking contact with family, friends, and acquaintances because they did not want to be confronted with awkward questions and remarks about their family member with a mental illness or their family anymore: "I often had that [avoiding social events]. You know, that I had to go somewhere, but I just stayed at home, avoided difficult situations. You realise your world is getting smaller." (Rachell, mother, age 59 years); "I think you're subconsciously aware of it [that some people think you're bringing up your child wrongly] and so you already have your story ready. You've heard it all before of course" (Cally, mother, age 59 years). Members of the family of a person with mental illness also feared stigmatising reactions and the potential loss of relationships and friendships when it came to potential partners:

It's not really a problem, but of course it's never easy and I still come up against it sometimes. So you have something like, for example, l've got a girlfriend, should I tell her... I've been going out with her for 3 or 4 months... but she's never met my mother. And I do have a particular reason, for, like, putting it off as long as possible. And that, oh I don't know, feeling embarrassed, it's 
just the idea, I don't know actually. Let her get to know me first and if she likes me enough, then it won't make any difference anymore (John, son, age 25 years).

Negative treatment and exclusion were also commonly reported experiences of stigma by association. A number of participants noted that teenaged and adolescent family members of people with mental illness experience explicit stigmatisation and negative treatment because of their association with a stigmatised person: "Then I thought, well for goodness sake, he's the one with the problem and now I have to go to the psychologist [with him]. Especially because other people were judging and teasing me for it" (Mike, brother, age 29 years). Several participants felt themselves stigmatised by mental health professionals as well:

So I had to go on one of those visits to the psychologist or psychiatrist. I went more for my parents than for my sister because I couldn't do anything to help her anyway. So I went there, but I hated it. They started off by commenting on where we sat down, that my parents were very protective, and you had to talk about all sorts of personal things that you wouldn't normally talk about. I absolutely hated that and that's one of the reasons that I'm not letting my children take part in all sorts of national health studies and things like that. Ugh, I never went back again, (Jennifer, sister, age 45 years).

Some family members of people with mental illness recalled stigmatisation which took the form of being blamed for the onset or continuation of the mental illness of their family member: “My parents didn't know anything about it [mental illnesses]. They're from another generation. They were like, do you mean to say that we...[caused our grandson's mental health problems]. Yes, we felt ashamed too. Acceptance is a process" (Chris, father, age 47, years).

Lastly, several participants explained that direct burdens and day-to-day problems which occurred during mental health crises pushed their experiences of stigma by association into the background: “No, people definitely don't understand and there have been a few major rows about it [verbal stigmatisation]. I used to think, mind your own business, and l'd say it out loud later, but l'd had enough. At the time I had other things to worry about" (Ken, husband, age 54 years).

\section{Burden}

Eighty-seven percent of the participants in this qualitative study also reported experiencing family burden. 


\section{Objective burden}

The participants who had a family member with mental illness reported concrete problems and the effects these had on the family's household routine and everyday tasks, as well as other concrete ways in which their life was disrupted. Quarrels and tussles were reported frequently:

My mother was really upset that we argued so often at home, that we as siblings sometimes wanted him to go away and sometimes hit him. And then she was angry with us and that upset me, but, at the same time, I thought if he'd just act normally, then we wouldn't have to react like that (Brita, sister, age 30 years).

A few participants also experienced financial hardship. In some cases, the family member with mental illness had not been able to manage his/her finances and had incurred debts that he/she could not pay back. The participants supported this member of the family financially and were expected to cope with the financial and judicial problems at the same time: "Now we're trying to clear up the mess again, financial mess too because there are three debt collectors on the doorstep. It's taking months. It wears you out" (Yasmina, mother, age 59 years). Occupational limitations, missed career opportunities, and, consequently, financial hardship sometimes were occasionally mentioned by participants. One of them said that his wife had to stop working because of their son's illness: "My wife was a teacher and we reached the point where we decided she'd have to stay at home [and give up her job] and take care of him" (Bill, father, age 58 years).

\section{Subjective burden}

The participants spoke with great emotional intensity about the subjective burden their family member's mental illness had placed on them and the psychological distress engendered by being a family member of someone with mental illness. Having a family member with a mental illness was also very time-consuming for most participants, and they reported that it could lead to feelings of exhaustion: "Always making excuses, more excuses, always talking your way out of things, that's what you have to do. And never being able to rest, always being on the edge of my seat; even now, always ready for action, always alert." (Frieda, spouse, age 61 years), similarly:

You can't really manage the instability. You can't really live with it. Constantly asking if it's all right but making a point of never asking how they really feel, is exhausting. So in that sense it has taken its toll on me, on my energy and on my enjoyment of life. You're dealing with it almost 24 hours a day, even if you're not dealing with it (Lenny, spouse, age 45 years). 
Some siblings and parents noticed that the mental illness of a family member was not only time-consuming but also led to the other children in the household receiving less attention and that this change or disruption of family relationships sometimes led to feelings of guilt and anger. One participant explained that she felt forlorn because of the attention given to her sister with mental illness:

As a sister, you've got your own life, but suddenly nobody pays any attention to what you're doing anymore, because that's all going well. All the attention is focused on one problem, her illness, and you're not involved in it anymore and then you no longer have any real contact with your family [sigh]. You sort of accept it, but you're really always an afterthought (Jennifer, sister, age 45 years).

Another participant mentioned that he and his wife suddenly noticed that their attention was almost exclusively focused on the son who had a mental illness and that this was affecting their other two sons negatively:

There was a time that he [the son with a mental illness] was the only thing we could talk about. Everything revolved around that, everything. Suddenly I realised that the other two boys just weren't getting enough attention. That's a dangerous situation; everything was centred on him. His name came up in every sentence, even when the other boys asked me something; then I referred to his name in my answer. So they began to rebel against it. We should have recorded it and listened to it in the evening. We would have been thoroughly ashamed of ourselves (Chris, father, age 47 years).

Finally, feelings of helplessness and loneliness were also common among participants: "It's really tough, and you feel really isolated, even from the people around you. You think that nobody understands you anymore, but it isn't possible for people to understand, I realise that now" (Frieda, spouse, age 61 years).

\section{Coping}

In an effort to mitigate the effects of stigma by association and family burden, family members of people with mental illness used various problem- and emotion-focused coping strategies (Carver, 1997; Miller \& Major, 2000). Sometimes, family members used just a single coping strategy, but most used several coping strategies simultaneously (Miller \& Kaiser, 2001). 


\section{Problem-focused coping}

Many participants in our study reported using problem-focused coping strategies, and a number of them mentioned using selective disclosure as an active coping strategy to prevent stigma by association and its negative impact: "Being too open isn't good either, but I do think functional openness is important." (Jessica, mother, age 58 years), "After all, you do have to explain that it's not going so well and why you're not working so well. So, I did want to do something to create understanding and as a sort of explanation of why I can't cope for a while" (Lenny, spouse, age 45 years).

Coping strategies whereby participants tried to get emotional support, understanding, or empathy were reported frequently too: "You can discuss how it makes you feel, if you need help or something, if you need information, I don't know, do you need someone to listen?" (Frieda, spouse, age 61 years). The use of instrumental support, for example, information, help, practical support in daily life, and advice about what to do, was also regularly used as a coping strategy:

I think that parents have to get help and you can't do it all on your own. You have to follow the same rules and if you can't, for God's sake get some help or you'll end up getting divorced. You need to talk to somebody sometimes, with experts so that you really know what's going on. Professionals have to help people to look at the situation and work out how they can deal with it together. (Bill, father, age 58 years)

In addition, several participants started organising support systems: "Of course, I had to build up a support system because I had a job and all sorts of responsibilities" (Linn, mother, age 57 years), "[asked how he coped] Build up a network so that other people can take over responsibility, can take your place, can replace you." (Lenny, spouse, age 45 years).

\section{Emotion-focused coping}

Family members of people with mental illness used emotion-focused coping strategies and problem-focused coping strategies. Denial, behavioural disengagement, selfdistraction, acceptance, and venting were reported by participants.

"Accepting that the mental illness was real, stigmatisation did occur. and one had to accept and handle the stigma", was mentioned as a strategy for diminishing the impact of stigma by association and its associated psychological distress as well: "Acceptance is number one; you have to accept it. You can't change it anyway because that's not going to happen. You shouldn't make it too much your own problem." (John, son, age 25 years) and "Now he's been diagnosed, I can understand things better, it gives me more 
peace. I think because I can accept it more easily when he's slow sometimes now" (Linn, mother, age 57 years).

The participants described in detail how they used activities in an attempt to distract themselves from the stigmatising family situation. Frequently mentioned opportunities for distraction were going out and participants' workplaces: "There's not a good environment at home, so you have to create a comfortable feeling for yourself, you have to make something of it yourself. I found it at work, going out, with my friends." (Jennifer, sister, age 45 years), "Work kept me going all that time. It was like my comfort blanket. I'd rather go to work than to the supermarket where you see everyone you know and they avoid you like the plague." (Frieda, spouse, age 61 years), and "I could relax at work and honestly, there's nothing worse than people who can only relax at work. That means their home environment isn't a safe haven anymore" (Ken, spouse, age 54 years).

Denial and rejection of the reality of the stigmatising situation were other tactics used by participants: "We had a period when we ignored everything, we were in denial, a bit like keeping it secret, and then, if you went out, don't talk about it!" (Chris, father, age 47 years). Several participants who had a family member with mental illness gave up on attempts to cope with stigma by association and its consequences. This approach was based mainly on behavioural disengagement: "I'm very down-to-earth about it. It's not my problem, so I distance myself from it. You place the problem clearly with him or her, not my problem" (John, son, age 25 years). The participants also reported avoiding situations in which they were likely to be expected to talk about their family member's mental health condition: "You don't go to parties anymore, you don't go outside with her, you only go out to do the shopping. Actually, you make it so that she becomes isolated too" (Ken, spouse, age 54 years).

Lastly, some participants indicated that they coped with stigma by association and burden by venting their emotions and discharging their feelings of emotional distress:

So then, I had an appointment with a psychiatrist. He didn't have to say anything, I tell you, I talked for an hour and a half and I was so angry and said to his face, 'You just sit there and I'll do the talking' and in an hour and a half, I let it all out and said 'I never want to see you again.' That's what I did. I spent an hour and a half ranting, crying and sobbing, swearing, talking, and then I left, and I've never seen him since (Frieda, spouse, age 61 years).

Humour, religion, positive reframing, and substance use were indicated as possible coping strategies in earlier studies (Carver, 1997), but these coping strategies were not explicitly reported by the participants in our study. 


\section{Contact}

Most family members in this study kept in regular contact with the member of their family who had a mental illness. They were in contact in person, by telephone, or by email (Van Erp et al., 2009), typically on a daily or weekly basis. However, some participants acknowledged they had little contact with that family member. They stated this was primarily a consequence of that family member's previous or current behaviour or because of quarrels and tussles they have had with that family member. Two participants reported that they did not wanted to be associated with that family member anymore because of his/her behaviour, his/her illness, or its stigmatising effect; they had withdrawn almost entirely from the relationship and minimised contact.

\section{DISCUSSION}

The participants in this study reported a range of forms of stigma by association including negative treatment, not being taken seriously, being blamed, social exclusion, as well as alterations in family relationships and friendships. These experiences of stigma by association are similar to those reported by Lefley (1989), who found that stigma transferred from people with mental illness to their family, resulting in the erection of social barriers, isolation of the family and a diminishing of its reputation, as well as jeopardising of social relationships with family, friends, and neighbours. Some participants noticed that they reduced the size of their social network and avoided social events because they could no longer handle the distress caused by being confronted with stigmatising behaviour and remarks directed against them or their family member with mental illness. These findings are in line with those of Tsang et al. (2003), who noticed that some family members of people with mental illness avoided social events and sacrificed social relationships to conceal the mental illness of a family member. Some participants mentioned being blamed for the onset or continuation of a family member's mental illness as a specific example of stigma by association. This is in accordance with Corrigan and Miller (2004), who found that family members of a person with mental illness were blamed for the onset of that person's mental illness, held responsible for relapses, and socially excluded as a result.

The participants also described various manifestations of family burden including quarrels, financial hardship, and missed career opportunities. The participants mentioned that taking care of a person with mental illness was very time-consuming as well. Similar burdens (e.g., social isolation, time-consuming activities, financial hardship, altered family relationships, and disruptions of household functioning) were found among family members of people with mental illness by Tsang et al. (2003) and Lefley (1989). 
It was not usually possible for the participants to determinate exactly which part of the negative impact (e.g., psychological distress, diminished quality of life) was related to stigma by association and which was related to family burden; most of the time, a combination of the two seemed to be involved. The participants also reported that, during a mental health crisis, they focused on surviving and handling the crisis and associated burdens and paid much less attention to coping with experiences of stigma by association. During these crises, the direct burden and day-to-day problems had to be handled, and this seemed to push stigma by association into the background. It seemed that, during a crisis, the participants could no longer deal with all experiences, problems, and burdens simultaneously and had to prioritise. However, mental health crises can vary substantially in type and severity, and therefore, the participants' experiences and reactions during these crises may vary across crises and individuals.

The participants used both problem-focused coping strategies and emotion-focused coping strategies to diminish the negative impact of stigma by association and family burden. This is in line with the findings of Eaton et al. (2011), who reported that some family members of people with mental illness used emotion-focused, avoidance-based coping strategies when they had to deal with the stressor and its consequences, whereas others used problem-focused coping strategies and made efforts to reduce the frequency or intensity of the stressor and manage its impact. Problem-focused coping strategies used by participants in our research were mostly aimed at obtaining social, instrumental, or emotional support. The most frequently used problem-focused coping strategies reported by participants were selective disclosure, organising support systems, trying to get empathy, talking to professionals and asking for professional support. Organising support systems was the main factor referred to when discussing active handling of the burdensome family situation. Selective disclosure was also used as an active coping strategy by participants, as a way of creating understanding and increasing support. This finding is similar to Bos et al. (2009), who found selective disclosure was used by mental health service users as a way of generating social support and diminishing stigmatisation. Emotion-focused coping strategies used by participants seemed to be aimed at evading or distancing oneself from stigmatised conditions and burden. Specific emotion-focused coping strategies reported by participants included accepting a stigmatising situation, being reserved when talking about the family member with mental illness, disengaging or withdrawing from the family member with mental illness or from the stigmatising situation, venting, distracting themselves by going out, taking refuge in work, or visiting friends. The participants, especially spouses, noticed that taking refuge in work as a form of self-distraction was an important signal that things were going wrong at home; however, this also seemed to be a generally accepted way of coping, and was seen as an easy way out of the stigmatising and burdensome family situation. Emotion-focused coping strategies seemed, at least some of the time, to reduce the intensity of negative and distressing emotions resulting from 
stigma by association and family burden. This is in line with the findings of Clarke and Tanya (2009) who reported that short-term stress reduction and improvements in patients' quality of life were more strongly associated with emotion-focused coping strategies, whereas problem-focused coping strategies were associated with longer-term improvement. The participants in our study seemed to use both types of strategy separately and simultaneously at different points, a finding in line with Stutterheim (2011), who reports that human immunodeficiency virus-positive participants combined several coping strategies to mitigate the negative impact of stigmatisation.

During interviews, several participants drew attention to the specific challenges faced by siblings of people with mental illness, stating that siblings generally had or felt less responsibility for caregiving and rehabilitation of the person with mental illness than did parents or spouses. This is consistent with the findings of Seeman (2013), who reported that siblings of patients with schizophrenia were an important component of the patient's social network but mostly did not take an active part in caregiving until their parents could no longer cope alone. Our participants also reported that most people with mental illness claimed a lot of attention at the expense of their siblings. Some siblings in our study found this extremely disruptive to family relationships and household functioning. Lefley (1989) reported similarly that the attentional focus on people with mental illness, together with the time spent on caregiving, often led to neglect of other family members. Several siblings in our study found taking part in family therapy stigmatising and burdensome. In particular, talking about personal feelings and thoughts in the presence of their parents or to "complete strangers" (i.e., mental health professionals) was especially challenging for some siblings during puberty and adolescence. Some of them felt forced to do so, and this caused shame and anger. They may have felt themselves stigmatised by their siblings' mental illness, and this could have had a negative effect on their involvement in the treatment processes and family therapy. Consequently, we found that the risk of losing siblings' involvement and support within the household of people with mental illness is not imaginary (Corrigan et al., 2004; Seeman, 2013). Good communication within the family, attention to siblings' emotions and personal needs, clear expectations, as well as family roles may be needed to keep siblings involved in the family situation and supportive of therapy. Specific attention should be paid to the loss, grief, frustration, loneliness, shame, and anger associated with having a sibling with mental illness (Ewertzon et al., 2012). Mental health professionals and parents should invite siblings to special events and psychoeducational group discussions on a strictly voluntary basis and develop these events in partnership with the siblings of people with mental illness. 


\section{Study strengths and limitations}

Our study has both strengths and limitations. Most importantly, this study is among the first to examine stigma by association, family burden, and coping strategies together. The rigour of our study is another strength; we ensured research quality, reliability, and validity by using a semi-structured protocol consisting of open-ended questions and follow-up probes to structure interviews. The involvement of people with mental illness and their family members in the development of the interview protocol - enriching the protocol through partnership - was another strength of this study. Lastly, the rigour of our study was further improved by using digital voice recorders and verbatim transcription to construct extensive "thick" descriptions, recording the decision trail transparently throughout the data analysis process and using proven, validated qualitative data analysis software.

However, some limitations to our study must also be mentioned. Caution should be applied in generalising the results to other populations because this was qualitative research. Another limitation is that most participants were recruited via self-help and support groups, which may have resulted in a sample biased towards highly involved individuals. The participants in our study may have been better equipped than most family of people with mental illness to cope with experiences of stigma by association because most were receiving support from mental health professionals; this study may, therefore, have underestimated stigma by association and its wider impact. The open coding in this study was mainly done by the first author, whereas interrater reliability could have enhanced the rigour of the study. Lastly, the participants reported not only their present but also their past experiences; their recollection of past and present emotions may not have been accurate, and their interpretation of past events and experiences may have changed over time.

\section{Conclusion and implications}

We have found that participants suffer as a result of stigma by association and family burden. Social exclusion, time-consuming caregiving activities, being blamed, not being taken seriously, and exhaustion were conspicuous features of stigma by association and family burden for the family members of people with mental illness. Participants in our study used problem-focused and emotion-focused coping strategies to cope with their experiences of stigma by association and family burden. They used both types of strategy in combination and separately. However, during mental health crises participants seemed to concentrate almost exclusively on dealing with the immediate burden of the situation, rather than worrying about stigma by association. Lastly, it seems that siblings need specific attention if they are to remain involved with and supportive of their family, particularly during puberty and adolescence. Our findings suggest that services 
should be provided for the families of people with mental illness to address these problems. When one is confronted with stress that exceeds one's ability to cope, for example, the onset or relapse of a family member's mental illness, other family members and mental health professionals should provide emotional support and assist with coping strategies to enable one to deal with the new family situation. Social support and the efforts of participants to help their family members with mental illness should also be recognised, acknowledged, and reinforced by extended family members, community members, and mental health professionals. There should also be services and facilities to provide respite from caring for a family member with mental illness. Lastly, particular attention should be paid to the personal needs and emotions of siblings of people with mental illness; they should be encouraged to attend psycho-educational group discussions and events by self-help groups, although attendance should be on a voluntary basis. We recommend that future research investigate the relationships among family roles, stigma by association, family burden, and coping more extensively. We advocate future research to include younger family members, aged between 12 and 18 years. We further recommend community-based participatory research into the specific relationships involving stigma by association, family burden, and their impact. Such research should be done through a strong, carefully considered partnership between researchers and the families of people with mental illness. Lastly, we suggest further quantitative research to focus specifically on the distinction between stigma by association and family burden as well as potential differences between strategies used to cope with stigma by association and family burden. 



\section{CHAPTER 5}

\section{HARMFUL EFFECTS OF STIGMA BY}

ASSOCIATION AND FAMILY BURDEN AMONG

FAMILY MEMBERS OF PEOPLE WITH MENTAL

ILLNESS: THE MEDIATING ROLE OF COPING

This chapter has been submitted for publication in similar form as:

Van der Sanden, R. L. M., Pryor, J. B., Stutterheim, S. E., Kok, G, \& Bos, A. E. R. (2014).

Harmful effects of stigma by association and family burden among family members of people with mental illness: The mediating role of coping. 


\section{ABSTRACT}

When someone has a mental illness, family members share the experience of stigma. Past research has established that family members' experiences of stigma by association predict psychological distress and lower quality of life. The present study examined how the use of different coping strategies mediates the relationship between experiencing stigma by association and these negative outcomes. In addition, the mediating roles of coping strategies in the relationship between the perceived family burden (i.e., practical problems) of having a family member with a mental illness and these negative outcomes were examined. In this cross-sectional survey study, data from 503 participants were analysed. As in previous research, both perceived stigma by association and family burden were associated with greater psychological distress and lower quality of life. The use of some problem-focused coping strategies mediated the relationship between experiencing stigma by association and lowered quality of life, but not increased psychological distress. Using these strategies was related to reduced negative outcomes. The use of several emotion-focused coping strategies mediated the relationships between experiencing stigma by association to both increased psychological distress and lower quality of life. Using emotion-based strategies was most often related to enhanced negative outcomes. Somewhat different pattern of mediation were noted when examining the relationship of family burden to these outcomes. Implications for intervention development are discussed.

Keywords: Stigma by association, family burden, psychological distress, quality of life, coping, mental illness 
A stigma is a form of negative deviance that blemishes the identity and reputation of the person who bears the mark. It brands the bearer as someone to be avoided or socially excluded (Bos et al., 2013; Dovidio et al., 2000). Research suggests that people with mental illness, regardless of the specific psychiatric condition, are stigmatised more severely than those with other health conditions (Bos et al., 2009; Corrigan \& Miller, 2004). Moreover, despite many public information campaigns intended to reduce the stigmatisation of mental illness, public images of people with mental illness remain very negative, and stigma has an enormous impact on the lives of people with mental illness (Bos et al., 2009; González-Torres et al., 2007). As such stigma denies people with mental illness opportunities to work, to live independently, and above all, it constitutes one of their greatest barriers to a complete and satisfying life (Corrigan, 2005; Corrigan \& Shapiro, 2010).

Across many stigmatising conditions, research has shown that people who somehow are associated with stigmatised individuals also are stigmatised merely because of this connection (Bos et al., 2013; Hebl \& Mannix, 2003; Neuberg et al., 1994; Pryor et al., 2012). In this regard, many people experience stigma because of their associations with family members who have a mental illness (Angermeyer et al., 2003; Corrigan \& Miller, 2004). This phenomenon is called courtesy stigma or stigma by association (Bos et al., 2013; Goffman, 1963; Neuberg et al., 1994; Pryor et al., 2012) and it may represent a significant source of stress for people whose family members have a mental illness (Tsang et al., 2003; Werner et al., 2012). In addition to their experiences of stigma by association, family members of people with mental illness also experience stress stemming from a range of practical struggles that constitute family burden; they experience financial problems, worries about the patient, time consuming activities associated with caring and seeking treatment for the people with mental illness, missed career opportunities, and family quarrels (Farina et al., 1992; Judge, 1994; Tsang et al., 2003).

In conceptualising the potential harmful impact of stigma by association and family burden upon people who have family members with mental illness, psychological distress represents one part of a matrix of inter-related outcomes. Other outcomes include physical health, social relationships, and environmental factors. Psychological distress as such represents the negative mental health state recognisable by symptoms as anxiety, depression, negative affect, and loss of behavioural and emotional control (Veit \& Ware, 1983), whereas quality of life represents someone's perception of one's position in life within the systems and community in which one lives, but also in relation to one's expectations, standards and goals (Skevington, Lotfy, \& O'Connell, 2004). Various studies have shown that stigma by association and family burden can be major sources of psychological distress and diminished the quality of life of family members of people with mental illness (Bos et al., 2013; Lefley, 1989; Rüsch et al., 2009; Van der Sanden et al., 2014; Werner et al., 2012). 
In seeking to mitigate the negative impact (e.g., psychological distress and diminished quality of life) of perceived experiences of stigma by association and family burden, family members of people with mental illness use various coping strategies (Lazarus \& Folkman, 1984; Lefley, 1989; Werner et al., 2012). Lazarus and Folkman (1984, pp. 141) defined coping as "constantly changing cognitive and behavioral efforts to manage specific external and internal demands that are appraised as taxing or exceeding the resources of the person". Lazarus and Folkman (1984) also made a distinction between problem-focused coping strategies and emotion-focused coping strategies. Problemfocused coping strategies aim on targeting, changing, or modifying the fundamental cause of the stressor, and appear to mitigate the negative impact of the stressor and to facilitate the recovery of one's function, whereas emotion-focused coping strategies focus on emotion-regulation and managing the negative emotions associated with the stressful condition, and are often seen as less adaptive (Carver, 1997; Eaton et al., 2011; Lazarus \& Folkman, 1984; Van der Sanden et al., 2014). Moreover, Moos and Holahan (2003) who examined coping styles, coping skills, and their adaptive function in more detail, showed most emotion-focused coping strategies to have a negative impact on one's adaptability to stressors, whereas problem-focused coping strategies were found to have a positive effect on participants' adaptability to stressful conditions or situations.

Van der Sanden and colleagues (2014) conducted a qualitative study about coping with stigma by association and family burden among twenty-three family members of people with mental illness. This study revealed that both stigma by association and family burden had detrimental effects the psychological health and perceived quality of life of these family members. Family members of people with mental illness in this study reported using a combination of different coping strategies, either serially or simultaneously. The use of problem-focused strategies such as seeking emotional and instrumental support through selective disclosure to others who might help typically led to less psychological distress and better quality of life. Also, the use of the emotion-focused strategy of acceptance helped to relieve the psychological distress related to sharing the stigma. However, the use of other emotion-focused strategies such as denial, behavioural disengagement, self-distraction, and venting typically produced only shortterm relief and often exacerbated negative outcomes. The current research examined these same issues using a quantitative methodology.

In-depth knowledge about the associations between stigma by association, family burden, their negative impact, and coping strategies is important with regard to the establishment and implementation of effective intervention techniques among family members of people with mental illness. For this reason, the present study examined the effects of fourteen problem-focused (i.e., active coping, use of emotional support, use of instrumental support, planning, using humour) and emotion-focused coping strate- 
gies (self-distraction, denial, substance use, behavioural disengagement, venting, positive reframing, acceptance, turning to religion, self-blame) on the associations between stigma by association and family burden on the one side, and psychological distress and quality of life on the other side. We tested the following hypotheses: First, it was hypothesised that experiences of stigma by association and family burden among family members of people with mental illness would independently predict increased levels of psychological distress, and diminished quality of life. Secondly, we expected both problem-focused and emotion-focused coping strategies to mediate the effects of stigma by association and family burden upon psychological distress and quality of life. On the basis of previous research (Carver et al., 1989; Chronister, Chou, \& Liao, 2013; Chronister, Chan, Sasson-Gelman, \& Chiu, 2010; Eaton et al., 2011; Moos \& Holahan, 2003; Van der Sanden et al., 2014), we expected that coping strategies such as active coping, use of emotional support, use of instrumental support, planning, using humour, acceptance, turning to religion, and positive reframing would mitigate the effects of stigma by association and family burden on psychological distress and quality of life. On the other hand, we expected that coping strategies such as self-distraction, denial, substance use, behavioural disengagement, venting, and self-blame would exacerbate the effects of stigma by association and family burden on psychological distress and quality of life.

\section{METHOD}

\section{Participants and procedure}

In October 2013, immediate family members of people with mental illness in the Netherlands were recruited from an online panel $(N=14,170)$ through email, asked whether they had a family member with mental illness, and if they were willing to participate in a self-report study on this topic. A positive response to this email was given by 6,840 panel members, and a random sample of 625 cases was drawn from these panel members. They were subsequently invited by email to participate in the survey, and a reminder was sent four days after the initial invitation. Five hundred and three out of the 625 invited panel members completed the survey yielding a response rate of $80.3 \%$. Informed consent was obtained, and participants were given points that could be exchanged for discount coupons upon survey completion. The study was approved by the Ethics Committee at Maastricht University's Faculty of Psychology and Neuroscience.

Two hundred and twelve participants were men and 291 women. Ages ranged from 18 to 85 years $(M=45.4 ; S D=13.4)$. In terms of level of education, $22.1 \%$ had a low (i.e., elementary school or lower vocational training), 39.8\% medium (i.e., secondary or midlevel vocational training), and $38.1 \%$ high (i.e., college or university) level of education. Participants' relationships to their family members with mental illness varied: $34.4 \%$ 
were parents, $21.4 \%$ children, $22.7 \%$ siblings, and $21.5 \%$ spouses. From the participants, $44.1 \%$ lived together in the same household as their family member with mental illness, and $53.7 \%$ indicated that they had an active caregiving role towards their family member. The type of mental illnesses of participants' family members also varied: depression (36.8\%), ADHD/ADD (21.2\%), autism (19.2\%), anxiety disorder (15.8\%), bipolar disorder (11.0\%), personality disorder $(9.0 \%)$, schizophrenia or psychotic disorder $(6.8 \%)$, and other $(10.3 \%)$; because participants were allowed to select more than one mental disorder, the sum of the percentages exceeds $100 \%$.

\section{Measures}

Stigma by association was assessed using a 28-item Stigma by association scale (Van der Sanden et al., 2013) that measures participants' cognitive, emotional and behavioural reactions to being related to someone with a stigmatised condition. Items are rated on a five-point scale ranging from strongly disagree (1) to strongly agree (5). A higher score indicates greater stigma by association. Cronbach's alpha was .88 .

Family burden was measured with a 7-item Burden scale (Pryor et al., 2012; Pryor et al., submitted). Items (e.g.: "It caused financial hardships in our family.", "It is timeconsuming having a family member with a mental illness.") were rated on a five-point scale ranging from strongly disagree (1) to strongly agree (5). A higher score was considered indicative of greater family burden. Cronbach's alpha was .71.

Psychological distress was measured using the 18-item Mental Health Inventory (MHI; Veit \& Ware, 1983). The MHI has been used previously to examine the impact of stigma by association on psychological distress (Stutterheim et al., 2009; Stutterheim et al., 2011; Van der Sanden et al., 2013). The MHI measures positive affect (e.g., "Have you felt calm and peaceful?"), anxiety (e.g., "Have you felt tense or high-strung?"), depression (e.g., "Have you been in low or very low spirits?"), and behavioural control (e.g., "Have you felt emotionally stable?") over the four weeks prior to administration. Participants score items on a six-point scale ranging from none of the time (1) to all of the time (6). A higher score indicates more psychological distress. Cronbach's alpha was .94 .

Quality of Life was assessed using the WHO QOL-BREF. The WHO QOL-BREF is an abbreviated 26-item version of the WHO QOL-100 (Skevington et al., 2004; Xia, Li, Hau, Liu, \& Lu, 2012). It measures the experienced quality of life within participants over the last four weeks prior to administration. The WHO QOL-BREF contains one item from each of the 24 facets of quality of life included in the WHO QOL-100 (e.g., "How satisfied are you with the support you get from your friends?", "How satisfied are you with yourself?", "To what extent do you have the opportunity for leisure activities?", "How 
much do you need any medical treatment to function in your daily life?"), plus two items from the general facet on the overall quality of life and general health. Items were rated on a five-point scale ranging from none of the time (1) to all of the time (5). A higher score was considered indicative of greater quality of life. Cronbach's alpha was .94.

Coping was assessed with the 28-item brief Coping Orientation to Problem Experience scale (brief COPE scale; Carver, 1997). The brief COPE scale is a brief form of the previously published COPE-inventory (Carver et al., 1989), which has proven to be useful in health-related research (Carver, 1997). The scale was administered to assess participants' problem-focused and emotion-focused coping strategies. The scale has fourteen subscales and measures participants' coping strategies (i.e., active coping, use of instrumental support, use of emotional support, planning, and humour being problemfocused coping strategies, and self-distraction, denial, substance use, behavioural disengagement, venting, positive reframing, acceptance, turning to religion, and selfblame being emotion-focused coping strategies) (Eaton et al., 2011; Hassan et al., 2006; Nydegger et al., 2011). The brief COPE scale has two items per subscale. Items were rated on a four-point scale ranging from I have not been doing this at all (1) to I have been doing this a lot (4). Cronbach's alpha for the brief COPE scale was .75; Cronbach's alpha's for the separate coping subscales differed between .70 and .90, except for the subscales "humour" ( $\alpha=.57)$ and "active coping" $(\alpha=.60)$.

Demographic and background variables as age, gender, educational attainment, marital status, ethnicity, and familial relation (i.e., mother, father, brother, sister, son, daughter, spouse) were also assessed.

Descriptive statistics, correlational analyses, and multiple mediator analyses with a 95\% bias-corrected bootstrap confidence interval based on 10,000 bootstrap samples (Hayes, 2013; Preacher \& Hayes, 2004), were used to analyse the data.

\section{RESULTS}

Stigma by association and family burden were positively correlated with psychological distress $(r=.39, p<.001 ; r=.32, p<.001)$ and negatively correlated with quality of life $(r=-.36, p<.001 ; r=-.34, p<.001)$. These correlations, along with the means and standard deviations for the primary study variables, are presented in Table 5.1. 
Table 5.1 Means, standard deviations, and intercorrelations for main study variables

\begin{tabular}{lllllll}
\hline Variable & $\mathrm{M}$ & SD & 1 & 2 & 3 & 4 \\
\hline 1. Stigma by association & 1.66 & .60 & -- & $.40^{* * *}$ & $.39 * * *$ & $-.36^{* * *}$ \\
2. Family burden & 2.58 & .61 & -- & $32^{* * *}$ & $-.34^{* * *}$ \\
3. Psychological distress & 2.87 & .78 & & -- & $-.79 * * *$ \\
4. Quality of life & 3.60 & .61 & & & -- \\
\hline
\end{tabular}

$* p<.05,{ }^{* *} p<.01, * * * p<.001$

Multiple regression analyses that simultaneously assessed the relationships of stigma by association and family burden upon psychological distress respectively quality of life were also conducted. Results indicated the two predictors explained $18.6 \%$ of the variance in psychological distress $\left(R^{2}=.19, F(3,500)=57.29, p<.001\right)$ and $17.3 \%$ of the variance in quality of life $\left(R^{2}=.17, F(3,500)=52.12, p<.001\right)$. It was found that stigma by association predicted psychological distress $(=.311, p<.001)$ as did family burden $(\beta$ $=.200, p<.001)$. Stigma by association $(\beta=-.266, p<.001)$ and family burden $(\beta=-$ $.230, p<.001$ ) also predicted quality of life. Both predictors (i.e., stigma by association and family burden) remained significant in these simultaneous analyses, as such both account for a unique variance in the outcomes of psychological distress and quality of life. These findings confirmed our first hypothesis.

The mediating roles of fourteen different coping strategies ( 5 problem-focused and 9 emotion-focused strategies) are examined in this research. Multiple parallel mediation analyses were conducted with a $95 \%$ bias-corrected and accelerated confidence interval (Hayes, 2013; Preacher \& Hayes, 2004). In these analyses, we examined whether the set of variables represented by the fourteen coping strategies mediates the relationship of stigma by association (respectively family burden) upon psychological distress (respectively quality of life). We also examined to what extent specific coping strategies mediate these relationships. Following the procedures developed by Preacher and Hayes (2004), we not only analysed the total effect of independent variables and mediators on dependent variables (c path) and the direct effect of independent variables on dependent variables ( $c^{\prime}$ path), but also the relationships between independent variables and mediators (a paths) and between mediators and dependent variables ( $b$ paths). Results are displayed in Figures 5.1 to 5.4. Confidence intervals, based on 10,000 bootstrap re-samples, containing zero are interpreted as not significant (Preacher \& Hayes, 2004; Hayes, 2013). In these analyses, several mediation effects were found for coping on the relationships between stigma by association respectively family burden and psychological distress and quality of life. Results are displayed in Tables 5.2 and 5.3. 
Table 5.2 Mediation Analyses for Coping Subscales on the Relationships between Stigma by Association respectively Family Burden and Psychological Distress

\begin{tabular}{|c|c|c|c|c|c|c|}
\hline & \multirow{2}{*}{\multicolumn{3}{|c|}{$\begin{array}{l}\text { Stigma by Association } \\
\mathrm{BCa} * 95 \% \mathrm{Cl}\end{array}$}} & \multirow{2}{*}{\multicolumn{3}{|c|}{$\begin{array}{l}\text { Family Burden } \\
\mathrm{BCa} * 95 \% \mathrm{Cl}\end{array}$}} \\
\hline & & & & & & \\
\hline & $\begin{array}{l}\text { Point } \\
\text { estimate }\end{array}$ & Lower & Upper & $\begin{array}{l}\text { Point } \\
\text { estimate }\end{array}$ & Lower & Upper \\
\hline Active Coping & -.0007 & -.0136 & .0069 & .0093 & -.0161 & .0402 \\
\hline Emotional Support & -.0057 & -.0305 & .0031 & -.0248 & -.0696 & .0110 \\
\hline Instrumental Support & .0042 & -.0042 & .0292 & .0144 & -.0310 & .0629 \\
\hline Planning & -.0026 & -.0225 & .0085 & .0248 & -.0063 & .0701 \\
\hline Humour & -.0158 & -.0502 & .0065 & -.0053 & -.0256 & .0032 \\
\hline Self-Distraction & .0640 & .0077 & .1234 & .0465 & .0108 & .0866 \\
\hline Denial & .0529 & .0034 & .1197 & .0481 & .0236 & .0872 \\
\hline Substance Use & .0664 & .0319 & .1355 & .0309 & .0110 & .0693 \\
\hline $\begin{array}{l}\text { Behavioural } \\
\text { Disengagement }\end{array}$ & .0529 & .0034 & .1197 & .0991 & .0582 & .1528 \\
\hline Venting & .0607 & .0168 & .1123 & .0541 & .0254 & .0979 \\
\hline Positive Reframing & .0313 & .0070 & .0716 & -.0033 & -.0283 & .0155 \\
\hline Acceptance & .0326 & .0092 & .0707 & -.0051 & -.0294 & .0128 \\
\hline Religion & -.0091 & -.0308 & .0001 & -.0055 & -.0224 & .0014 \\
\hline Self-Blame & .1180 & .0674 & .1801 & .0765 & .0423 & .1250 \\
\hline
\end{tabular}

* $\mathrm{BCa}=$ Bias corrected and accelerated bootstrapping confidence intervals.

Table 5.3 Mediation Analyses for Coping Subscales on the Relationships between Stigma by Association respectively Family Burden and Quality of Life

\begin{tabular}{|c|c|c|c|c|c|c|}
\hline & \multirow{2}{*}{\multicolumn{3}{|c|}{$\begin{array}{l}\text { Stigma by Association } \\
\text { BCa* } 95 \% \mathrm{Cl}\end{array}$}} & \multirow{2}{*}{\multicolumn{3}{|c|}{$\begin{array}{l}\text { Family Burden } \\
\mathrm{BCa} * 95 \% \mathrm{Cl}\end{array}$}} \\
\hline & & & & & & \\
\hline & $\begin{array}{l}\text { Point } \\
\text { estimate }\end{array}$ & Lower & Upper & $\begin{array}{l}\text { Point } \\
\text { estimate }\end{array}$ & Lower & Upper \\
\hline Active Coping & .0006 & -.0055 & .0120 & .0183 & .0008 & .0437 \\
\hline Emotional Support & .0112 & .0004 & .0407 & .0438 & .0151 & .0820 \\
\hline Instrumental Support & -.0028 & -.0239 & .0031 & .0339 & .0041 & .0682 \\
\hline Planning & .0018 & -.0063 & .0172 & .0234 & .0001 & .0519 \\
\hline Humour & .0079 & -.0099 & .0323 & .0021 & -.0052 & .0154 \\
\hline Self-Distraction & -.0499 & -.1036 & -.0105 & -.0498 & -.0873 & -.0196 \\
\hline Denial & .0188 & -.0201 & .0623 & -.0282 & -.0663 & -.0103 \\
\hline Substance Use & -.0468 & -.0964 & -.0211 & -.0222 & -.0513 & -.0071 \\
\hline Behavioural Disengagement & -.0924 & -.1610 & -.0470 & -.0695 & -.1136 & -.0398 \\
\hline Venting & .0024 & -.0364 & .0407 & -.0288 & -.0583 & -.0072 \\
\hline Positive Reframing & -.0218 & -.0517 & -.0040 & .0022 & -.0104 & .0202 \\
\hline Acceptance & -.0216 & -.0516 & -.0034 & .0035 & -.0083 & .0214 \\
\hline Religion & .0076 & .0000 & .0257 & .0045 & -.0012 & .0173 \\
\hline Self-Blame & -.0709 & -.1149 & -.0346 & -.0456 & -.0778 & -.0228 \\
\hline
\end{tabular}

${ }^{*} \mathrm{BCa}=$ Bias corrected and accelerated bootstrapping confidence intervals. 
In examining Tables 5.2 and 5.3, only those confidence intervals that are entirely above or below zero indicate significant indirect effects of the mediator. By this criterion, none of the problem-focused coping strategies significantly mediated the relationship between stigma by association and psychological distress (see Table 5.2). On the other hand, all of the emotion-focused coping strategies produced significant indirect effects, except religion. Some emotion-focused coping strategies such as self-distraction, denial, substance use, behavioural disengagement, venting, and self-blame were associated with higher levels of psychological distress. A possible implication is that use of these strategies seemed to make people more psychologically distressed. Alternatively, positive reframing and acceptance were associated with lower levels of psychological distress (see Figure 5.1).

Similarly, none of the problem-focused coping strategies significantly mediated the relationship between family burden and psychological distress (see Table 5.2). The emotion-focused strategies that produced significant indirect effects were all associated with higher levels of psychological distress: self-distraction, denial, substance use, behavioural disengagement, venting, and self-blame (see Figure 5.2).

Turning to the prediction of quality of life, some different patterns of mediation emerged (see Table 5.3). The problem-focused strategy of seeking emotional support significantly mediated the relationship between stigma by association and quality of life. Use of this strategy was related to higher levels of quality of life. Several emotionfocused strategies also produced significant indirect effects. Self-distraction, substance use, behavioural disengagement, and self-blame were associated with lower levels of quality of life (see Figure 5.3), whereas, positive reframing, and acceptance were associated with higher levels of quality of life.

Finally, in examining the potential mediators of the relationship between family burden and quality of life, we see that problem-focused strategies produced many more significant indirect effects (see Table 5.3). The problem-focused strategies of active coping, seeking emotional support, seeking instrumental support, and planning all produced significant indirect effects suggesting that the use of these strategies was related to higher levels of quality of life (see Figure 5.4). Relative to the other significant mediators, active coping had a weaker relationship to quality of life. Several emotion-focused strategies produced significant indirect effects and were associated with lower levels of quality of life: self-distraction, denial, substance use, behavioural disengagement, venting, and self-blame. 


\section{DISCUSSION}

The present study demonstrates that both perceived stigma by association and family burden are associated with higher levels of psychological distress and poorer quality of life. These higher levels of psychological distress and diminished quality of life among family members of people with mental illness are in line with earlier studies (Pryor et al., 2012; Östman \& Kjellin, 2002; Tsang et al., 2003; Van der Sanden et al., 2013; Van der Sanden et al., 2014). In fact, Östman and Kjellin (2002), and Van der Sanden et al. (2014) found that the lives of family members of people with mental illness are substantially disrupted, and their quality of life diminished as a result of their experiences of stigma by association and family burden.

In examining the roles of coping strategies as mediators of the associations of both stigma by association and family burden with psychological distress, a common pattern emerged. Problem-focused coping produced no indirect effects (i.e., they played no meditational roles) for either the relationship between stigma by association and psychological distress or the relationship between family burden and psychological distress. Further, most of the emotion-focused coping strategies that mediated the relationship between stigma by association and psychological distress, and the relationship between family burden and psychological distress represented complementary relationships (Zhao, Lynch, \& Chen, 2010). The implication is that use of these emotionfocused coping strategies resulted in worse psychological distress. The picture that emerges is one in which family members of people with mental illness experience psychological distress both from sharing the stigma (i.e., stigma by association) and the practical issues that arise from having a family member with mental illness, but most of the things they attempt to do in coping with these sources of psychological distress only exacerbate their distress. Fortunately, in the mediation analysis of the relationship between stigma by association and psychological distress, two emotion-focused strategies produced competitive meditational relationships (Zhao et al., 2010): positive reframing and acceptance. Use of these strategies was associated with lower psychological distress. Carver and colleagues (1989) suggest that positive reframing or construing a stressor in positive terms should intrinsically lead to a continuation or resumption of problem-focused coping actions. Another possibility is that the use of this strategy simply makes the source of stress seem less negative (a reappraisal). Acceptance also could reduce psychological distress in a number of ways. Acceptance that a family member has mental illness might be a precursor to providing social support to this family member, which in turn might improve his/her condition. Acceptance would seem particularly important if family member's mental illness is a permanent condition or unlikely to improve. 
Our analyses of the coping strategies that potentially mediate the relationship between stigma by association and quality of life, and between family burden and quality of life, showed problem-focused and emotional-focused coping strategies to have a mediational role. Findings suggest that people with a family member who has mental illness are likely to take action or engage in problem-focused coping strategies when their quality of life is threatened from these sources of stress. Thus, people might be described as seeking external solutions for what are perceived to be external problems. In this vein, seeking emotional support from others proved to be a significant competitive mediator in both quality of life analyses. A likely source of emotional support would be other family members who also share stigma by association concerns and the common family burden. In addition, other problem-focused coping strategies were significant competitive mediators on the relationship between family burden and quality of life: active coping, seeking instrumental support, and planning. The use of these strategies emphasises the practical nature of the problems that comprise family burden and the practical nature of the consequences that comprise quality of life. For example, reduced employment opportunities of a family member with mental illness could have a direct impact upon the financial resources of a family. However, whereas problemfocused strategies only emerged as mediators when examining quality of life outcomes, emotion-focused coping strategies emerged as significant mediators when examining both psychological distress and quality of life outcomes. Self-distraction, substance use, behavioural disengagement, and self-blame were all significant complementary mediators on the relationships between stigma by association and quality of life, and between family burden and quality of life. Use of these coping strategies was related to poorer quality of life.

These findings are consistent with findings from Fortune, Smith, and Garvey (2005) who, in their study among relatives of patients with schizophrenia, found that seeking emotional support and active coping were associated with less psychological distress, while coping through self-blame was related to increased psychological distress. Our results are also similar to those of Wahl, Hanestad, Wiklund, and Moum, (1999); they found that patients who combined emotion-focused coping strategies mostly reported more psychological distress and diminished quality of life, whereas patients who used problem-focused coping strategies generally reported less psychological distress and better quality of life.

Further explanation for the differences between the effects of the various coping strategies may lie in "the adaptiveness of coping strategies" (Kohn, O'Brien-Wood, Pickering, \& Decicco, 2003; Zeidner \& Saklofske, 1996). Adaptation refers to the degree to which someone successfully copes socially, physiologically, and psychologically with a stressor (Canam, 1993), and coping strategies are most times seen as predominantly adaptive or maladaptive (Moore et al., 2011). Adaptive coping strategies are considered to reduce 
stress levels, improve one's functioning and quality of life. Adversely, maladaptive coping strategies are assumed to reduce symptoms while maintaining and even strengthening the stressful situation or condition; they may temporarily mitigate the negative impact of the stressor, but they are mainly seen as counterproductive and ineffective in the long run (Moore et al., 2011). Coping strategies as self-distraction, denial, substance use, behavioural disengagement, venting, and self-blame may have a negative impact on one's adaptability to stressful and stigmatising situations, while active coping, use of emotional support, use of instrumental support, planning, positive reframing, and acceptance may have a positive effect on one's adaptability to these situations. Possibly, do family members of people with mental illness use [maladaptive] coping strategies as they aim to escape or avoid feelings of distress, what may eventually become a flight into inactivity, apathy, fantasy, or detachment, or may lead to feelings of hopelessness (Blum \& Silver, 2008; Eaton et al., 2011; Galaif, Sussman, Chou, \& Wills, 2003; Moore et al., 2011; Nakamura \& Orth, 2005; Pulla, Shatté, \& Warren, 2013; Reed, Kemeny, Taylor, Wang, \& Visscher, 1994). Coping strategies as active coping, use of emotional support, use of instrumental support, and planning on the other hand may actually attack and alter the stressor and thus improve one's personal situation and satisfaction with, for example, one's support from friends, income, or bodily appearance. These coping strategies may also make participants more aware of their possibilities to actively improve their personal and familial situation, what may reflect in their overall life satisfaction. This would be in line with Moos and Holahan (2003) who found most emotionfocused coping strategies to be potentially maladaptive coping strategies, whereas they found problem-focused coping strategies to be more adaptive coping strategies.

The findings of this quantitative study also support some of the predictions derived from our prior qualitative study, but conflict with others. Like the qualitative study, in the current research, the use of several emotion-focused coping strategies often exacerbated the relationships of stigma by association and family burden with psychological distress and quality of life. For example, when experiencing stigma by association or family burden, the use of behavioural disengagement and self-distraction strategies were associated with more psychological distress and diminished quality of life. Also parallel to our qualitative findings, acceptance was suggested as a remedy for the psychological distress related to stigma by association. The major difference in contrasting these two studies concerned the role of problem-focused coping. While the qualitative study suggested that using some problem-focused coping strategies might mediate the relationships between stigma by association and psychological distress or the relationship between family burden and psychological distress, the current quantitative study found no evidence for such mediation. What the quantitative study did reveal were indirect effects for using problem-focused strategies, such as seeking emotional support, in the relationships between both stigma by association and family burden with quality of life. 
What are we to make of the divergent findings of the prior qualitative and the current quantitative studies? One possibility speaks to potential shortcomings inherent with a qualitative approach. While in-depth interviews have the positive potential of exposing the nuances of how people react to complex social experiences like stigma by association, they rely upon and are limited to self-understanding of the relationships between experiences. People who have engaged in problem-focused coping strategies might believe that exerting such effort has resulted in their feeling less psychologically distressed. However, their self-knowledge lacks a control comparison of not having engaged in such strategies. This is of course one of the primary reasons to conduct quantitative studies like the current one. Another possibility is that psychological distress and quality of life are not separable in qualitative assessments of these constructs. Using the current quantitative methods we demonstrated that while psychological distress and quality of life are related constructs, they are separable and related to distinct patterns of prediction and mediation.

\section{Study strengths and limitations}

The relative novelty of research on coping with stigma by association and family burden among family members of people with mental illness is a primary strength of this study. The large number of participants is another, as is the broad range of mental illnesses involved. A final strength is the use of extended and advanced multiple mediation analyses and bootstrapping techniques. However, the generalisability of our results may be limited for several reasons. Firstly, we conducted a cross-sectional study and therefore no conclusions can be drawn about causal effects. Secondly, the use of self-reports could also lead to response bias as respondents were possibly more motivated or accustomed to disclosure than non-respondents (Donaldson \& Grant-Vallone, 2002; Fiske \& Taylor, 1991). Lastly, when generalising the results in this study, the relative low Cronbach's alphas of the subscales "humour" and "active coping" should be taken into account.

\section{Theoretical and practical implications}

The findings of our study have implications for both practice and theory. Given the relative novelty of research on coping with stigma by association and family burden among family members of people with mental illness, and especially of the detailed exploration of the fourteen coping strategies used in this study, the present study contributes considerably to the literature on stigma by association and family burden. Findings suggest that a stigma not only harms people with mental illness but also harms their immediate family members. Participants in this study have also shown active coping, use of instrumental support, use of emotional support, planning, positive reframing, and acceptance to be associated with a mitigated negative impact from stigma 
by association and family burden, whereas self-distraction, denial, substance use, behavioural disengagement, venting, and self-blame were related to an intensified negative impact from stigma by association and family burden. For this reason, information on the effectiveness of coping strategies should be actively promoted. Support in the use of these coping strategies should also be made readily available to family members. As such, mental health care professionals can play an important role in helping family members of people with mental illness enhance existing coping skills (Phelan et al, 2002; Van der Sanden et al., 2014). Mental health care professionals can facilitate family members to develop new coping strategies as well.

\section{Conclusion}

This research has been carried out to develop an in-depth insight in the experiences, processes, and consequences of stigma by association and family burden among family members of people with mental illness. Stigma by association and family burden were both shown to increase family members' psychological distress, and to diminish their quality of life. Several problem-focused coping strategies mitigated the negative impact of stigma by association and family burden, whereas most emotion-focused coping strategies were associated with an intensified negative impact. We recommend longitudinal research on the experiences and consequences of stigma by association, and to explicitly investigate the effect of problem-focused and emotion-focused coping strategies on the relationship between stigma by association, and psychological distress and quality of life, on the short term and in the long run. As family members strive to ameliorate their personal well-being, and given the emphasis placed by participants in this study on the importance of coping strategies to mitigate the negative impact of stigma by association and family burden, there seems to be an irrefutable need for education and support in the use and development of effective coping strategies. 


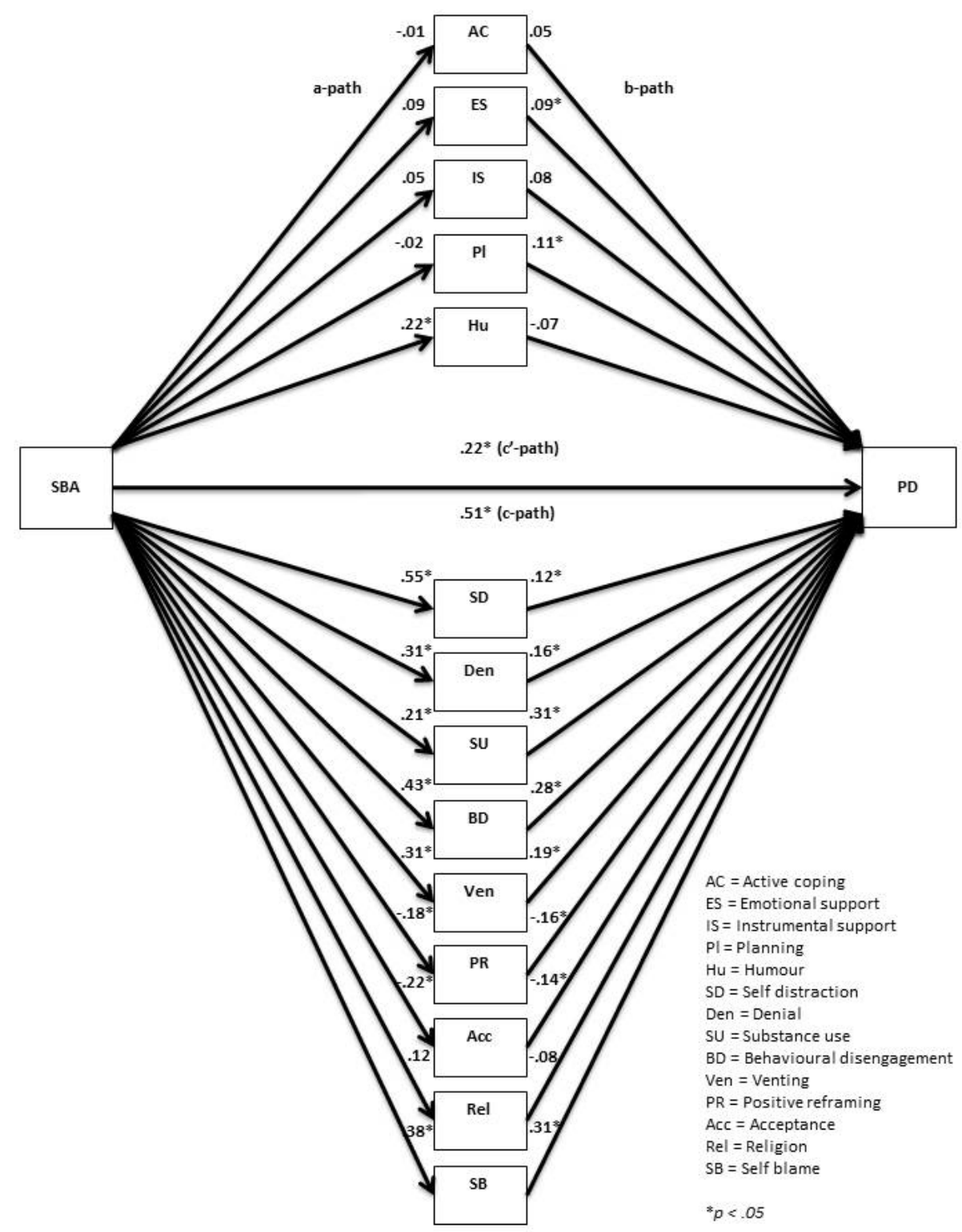

Figure 5.1 Mediation analyses from coping strategies on stigma by association (SBA) and psychological distress (PD) 


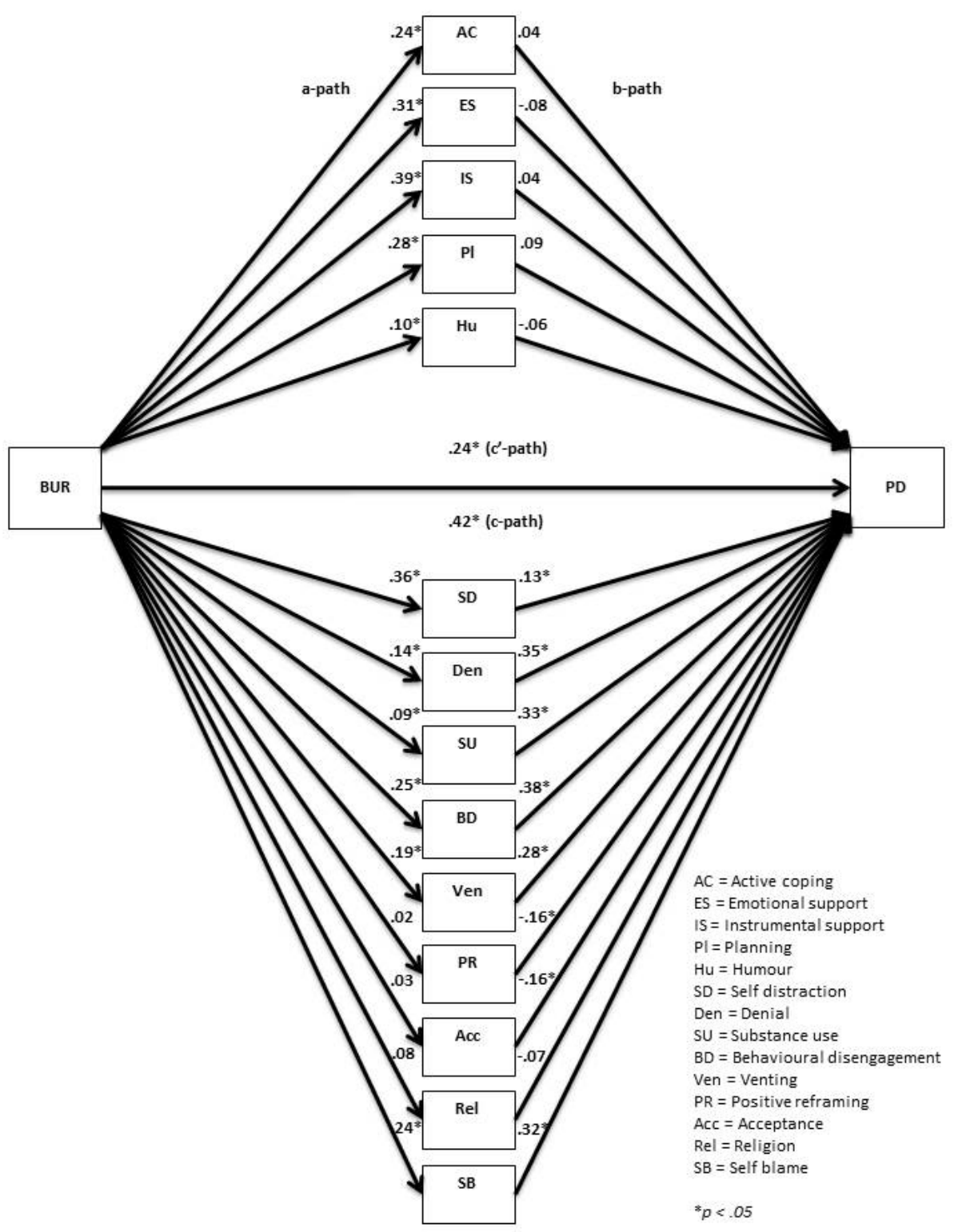

Figure 5.2 Mediation analyses from coping strategies on family burden (BUR) and psychological distress (PD) 


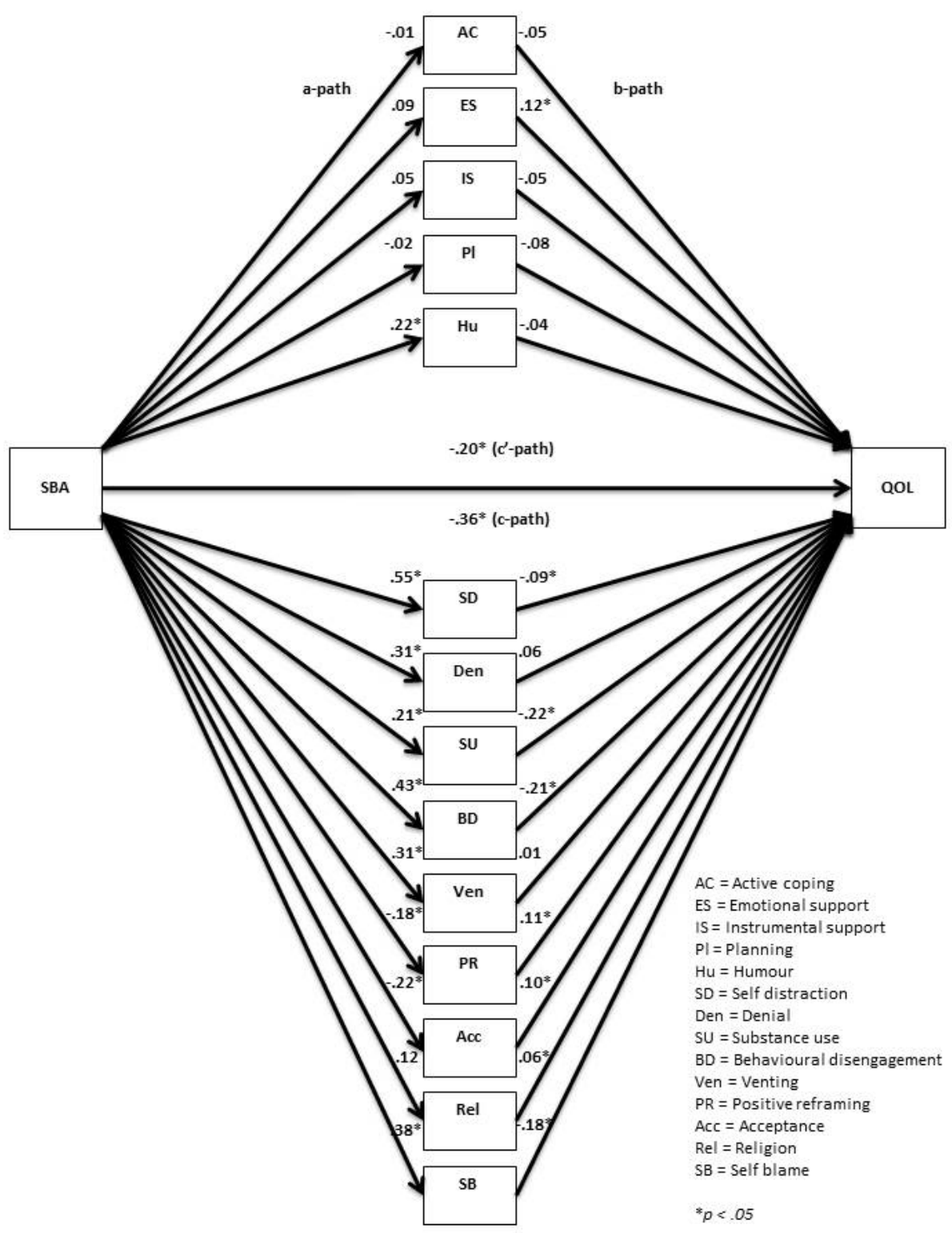

Figure 5.3 Mediation analyses from coping strategies on stigma by association (SBA) and quality of life (QoL) 


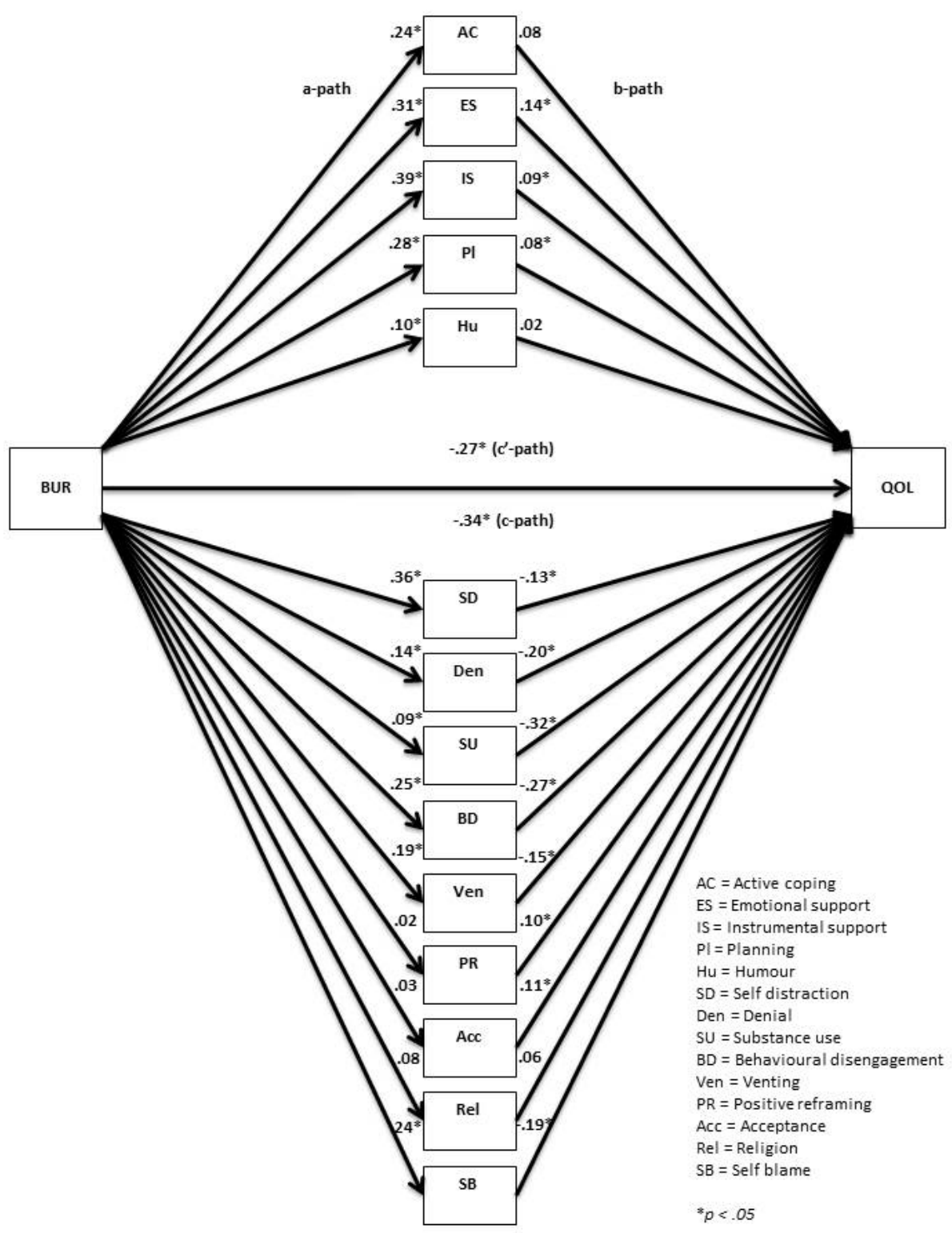

Figure 5.4 Mediation analyses from coping strategies on family burden (BUR) and quality of life (QoL) 

CHAPTER 6

GENERAL DISCUSSION 



\section{GENERAL DISCUSSION}

People with mental illness are regularly confronted with experiences of stigma. They are, however, not the sole recipients of stigma; their family members may be subjected to stigmatisation as well. This is known as courtesy stigma, family stigma, or stigma by association (Bos et al., 2013; Corrigan et al., 2006; Goffman, 1963; Lefley, 1989; Östman \& Kjellin, 2002; Pryor et al., 2012). In this dissertation, the social and psychological processes underlying, contributing to, and resulting from stigma by association have been examined. In particular, the studies presented in this dissertation investigated the manifestations and experiences of stigma by association among family members of people with mental illness, the consequences of stigma by association, the effect of several relevant family members' characteristics on experiences of stigma by association, and coping strategies employed by family members to mitigate stigma by association and its negative consequences.

In this last chapter, the findings of the studies reported in this dissertation are summarised and discussed in the context of the existing literature. Subsequently, this chapter reflects on the methodological strengths and limitations of the studies presented in this dissertation. Lastly, attention is paid to the practical implications of the studies and recommendations for future research are provided.

\section{Processes, experiences and consequences of stigma by association}

Stigmatisation is a complex social process related to stereotypical characteristics, prejudicial attitudes, and discriminatory behaviour towards people who are labelled as deviant and devalued, and people with mental illness belong to one of the most stigmatised groups in society (Brohan et al., 2010; Corrigan, 2005; Johnstone, 2001; Phelan et al., 2008; Stutterheim, 2011). However, not only are people with mental illness subjected to stigmatisation, people associated with them (e.g., family members, friends) can also be subjected to stigmatisation. This phenomenon is called stigma by association (Östman \& Kjellin, 2002).

In chapter 2, the relationship between public stigma (the perceived societal reactions to a stigma) and stigma by association was examined in a large sample of Dutch family members of people with mental illness $(N=527)$. The results of this quantitative study demonstrated that public stigma is positively related to the experience of stigma by association. As such, stigmatisation not only affects those who possess the stigmatised condition but also their family members (Bos et al., 2013). In essence, the public stigma was found to "spill over" from people with mental illness to their family members, and undesirable characteristics ascribed to people with mental illness (e.g., unstable, weird, unpredictable, unintelligent) were perceived to have been transferred to the previously 
non-stigmatised family members of people with mental illness. These results appear to be consistent with Pryor et al. (2012), who noted that people's stigma-relevant attitudes are related to how people regard family members of people with a stigmatised condition. This supports the notion that stigma by association is derived from a consciousness of general societal reactions to a stigma (public stigma).

Further, based on the findings reported in chapter 2, it appears that the process by which family members of people with mental illness are subjected to stigma by association parallels the process of public stigma. Like public stigma (Brohan et al., 2010; Corrigan, 2005; Phelan et al., 2008), the stigma by association to which family members of people with mental illness are subjected appears to be a social process that encompasses oversimplified conceptions, opinions, and images of family members of people with mental illness (stereotypes), negative attitudes that reinforce these stereotypes (prejudice), and negative behaviour (discrimination) towards family members of people with mental illness.

Family members of people with mental illness in the studies presented in both chapters 2 and 3 reported various experiences of stigma by association including blame, negative treatment, non-verbal expressions of discomfort, rejection, abandonment, social exclusion, and avoidance. These experiences were, in chapter 2 , found to be related to increased levels of psychological distress, reduced perceived closeness to their family member with mental illness, and diminished quality of life. In Chapter 3, the consequences were explored more contextually and included social consequences (e.g., disrupted relationships, alienation from family members and friends, altered family relationships, hampered development of new relationships, avoidance of social engagement, a reduced social network, and social isolation), psychological consequences (e.g., anxiety, psychological distress, brooding, irritability, frustration, anger, inner unrest, feelings of shame, undermined self-esteem, and diminished self-confidence), and health consequences (e.g., insomnia, fatigue, neck and shoulder pain, and headaches). These findings are corroborated by previous research conducted by Larson and Corrigan (2008), who, in their study, found that experiences of stigma by association have damaging effects on the lives of family members of people with mental illness. More specifically, Larson and Corrigan (2008) noted that family members worried about being blamed and had strained relationships with others, lower self-esteem, and a diminished quality of life.

Larson and Corrigan (2008) also reported underlying processes and experiences of stigma by association that are similar to those reported in chapters 2 and 3 of this dissertation. For example, in Larson and Corrigan's (2008) study, parents reported having been blamed for their child(ren)'s mental illness and having been accused by their communities of having poor parenting skills that have apparently led to the onset and 
continuation of their child(ren)'s mental illness. Larson and Corrigan (2008) further reported that, when regularly blamed, parents developed feelings of shame and guilt and that such feelings can lead to lower self-esteem and an impaired reputation within one's community which, in turn, can have a detrimental effect on well-being.

Chapter 3 of this dissertation also demonstrated how being associated with a family member with mental illness can pose a dilemma. The results suggest that family members of people with mental illness have to choose to either embrace and share the fate of their stigmatised family member by identifying with him or her, or refuse to share the discredit by avoiding, minimising, or rejecting their relationship with this family member. This dilemma can make family members of people with mental illness feel less closeness to their family member with mental illness and may reflect the exertion of psychological or physical distance in an effort to detach from the stigma carried by their family member with mental illness. This is an important finding as previous research has shown that the presence of supportive networks around people with mental illness reduces relapses into mental illness (Östman \& Kjellin, 2002). As such, reduced closeness may very well exacerbate the problems faced by people with mental illness.

Furthermore, a rather unexpected and troublesome finding of the qualitative study presented in chapter 3 was that over half of the participants had experienced stigmatising reactions from mental health professionals and public servants. Especially parents and spouses felt that they had unjustly been held responsible for the stigmatised condition, blamed for its onset, disqualified, ignored, and/or excluded from the treatment process. They felt "left out of the team" during treatment and often found the provision of information and communication from professionals to be inadequate. Moreover, these family members of people with mental illness frequently felt that professionals have demonstrated a lack of interest in, and insufficient recognition of, their family role and personal burden. They reported sometimes feeling as though they are viewed as a nuisance or as incompetent. What is noteworthy is that these kinds of experiences were perceived as even more harmful than experiences of stigma by association in interactions with others because family members of people with mental illness had prior expectation of professionals that were not in line with their experience. More specifically, they had thought that mental health professionals and civil servants would be empathic and supportive and were surprised and hurt when instead they were stigmatised, excluded, and ignored. Clearly, it seems that mental health professionals and civil servants are no less susceptible to stereotyping, prejudice, and discriminatory behaviour than the general public. Similar experiences of this particular form of stigma by association have been found previously in studies by Angermeyer et al. (2001) and by Östman and Kjellin (2002). Angermeyer et al. (2001) noted that mental health services and psychiatry are important sources of stigma toward family members of people 
with mental illness, while Östman and Kjellin (2002) reported that family members regularly had felt inferiority to mental health professionals in conversations.

\section{Relevant family members' characteristics and stigma by association}

The effects of familial relationship, co-residence, gender, and the belief that mental illness is hereditary on the relationships between stigma by association, psychological distress, and perceived closeness were also explored in chapters 2 and 3.

\section{Familial Relationship}

People with mental illness have a variety of immediate and extended family members and within families, each member has certain roles and certain characteristics. Consequently, each relationship within a family - whether it is between mother and child or father and child or between siblings - is unique.

In the study presented in chapter 2, the experiences and negative impact of stigma by association appeared to be less profound for extended family members than for immediate family members. Chapter 3 showed that parents and spouses are often held personally responsible by the general public for the onset and continuation of their family member's mental illness. Consequently, the parents and spouses in this study often reported encountering stigma by association manifest as blame, as well as avoidance and negative treatment. In contrast, children and siblings were rarely held responsible for their family member's mental illness. This is fairly logical given that children and siblings tend to have fewer responsibilities with regard to caregiving than parents and spouses and thus are less frequently reported being held responsible for the stigmatised condition. This is an important finding given that previous research has demonstrated that when a person is considered personally responsible for his or her stigmatised condition, stigmatisation is more likely (Bos et al., 2008; Dijker \& Koomen, 2003; Stutterheim, 2011). Extrapolating from those results, one could then contend that the degree to which a mother, father, or spouse is held responsible for the stigmatised condition of their family member with mental illness impacts the degree to which they experience stigma by association. That parents and spouses are more frequently held responsible for a family member's mental illness than siblings or other family members may be because parenthood, marriage, and partnership are perceived as voluntary choices while children and siblings do not choose their parents and siblings. Nonetheless, the children and siblings of people with mental illness that participated in the study presented in chapter 3 reported being seen as potentially deviant people and having experienced rejection, abandonment, and fear of contamination. Additionally, children of parents with mental illness reported feeling shame and guilt, as well as a conflict of loyalty, as a result of their experiences of stigma by association. On the one hand, they loved their parent with mental illness but, on the other hand, they did not 
want to be identified as being connected with the stigmatised condition of this parent (Romijn, De Graaf, \& De Jonge, 2010; Vanhauwaert, 2010). As such, children of a parent with mental illness were often inclined not to talk with others about their parent with mental illness, which, as has been demonstrated by others, may evoke feelings of social isolation and yield less social support (Van Santvoort, Hosman, Van Doesum, \& Janssens, 2014).

Furthermore, chapter 3 demonstrated that siblings of people with mental illness have unique stigma by association and family burden experiences, and thus experience unique consequences of stigma by association and family burden. This was reported to be especially the case during puberty and adolescence. Often, they found participating in more or less obligatory family counselling or family therapy during their youth to be very stigmatising. Additionally, some participants reported trying to conceal their familial relationship to their brother or sister with mental illness, particularly in their puberty and adolescence, when one is more easily influenced by peers than by parents. Adolescents and teenagers tend to show strong in-group favouritism (Banaji, Baron, Dunham, \& Olson, 2008; Eldering, 2006) and this in-group preference may make siblings more sensitive to perceived experiences of stigma by association. Additionally, participants who were siblings of someone with mental illness reported feeling, at some point in time, animosity towards their sibling as he or she claimed more attention, altered familial relationships, and disrupted household functioning.

\section{Co-residence}

Family members who lived together with their family member with mental illness, especially siblings, noted that co-residing exacerbates experiences of stigma by association and psychological distress. Family members also noted that co-residing with a family member with mental illness affected their social contacts and relationships with others.

Having a family member with a mental illness is concealable thus family members often face a dilemma of disclosure (Quinn \& Chaudoir, 2009). On the one hand, disclosure may evoke stigma by association and that can have a negative impact on the psychological well-being of family members of people with mental illness. On the other hand, disclosure has been found to enhance social, financial, or emotional support, and diminish psychological distress (Bos et al., 2009; Pachankis, 2007; Verhaeghe, Bracke, \& Bruynooghe, 2008). Previous research suggests that selective disclosure of a stigmatised condition can limit stigma experiences (Bos et al., 2009). However, even partial concealment of one's relationship to a person with mental illness is very difficult in circumstances whereby one co-resides with one's family member with mental illness and the findings presented in chapter 3 indeed suggest that co-residence plays a specific and important role in siblings' experiences of stigma by association. Especially in 
adolescence and puberty, siblings appeared to be very concerned that others would find out that they are related to a person with mental illness and stigmatise them. Given that relationships between siblings are not as commonly known by peers as relationships between parents and children or between spouses, concealment was considered a viable option at that time in their lives. It was also considered an appealing option in adolescence and puberty when one is more sensitive to how one is viewed by peers (Slot \& Van Aken, 2010). As such, participants reported taking great efforts to keep their familial relationship to a person with mental illness concealed and being anxious that they would be unsuccessful because of co-residence.

\section{Gender}

Earlier research by Farina (1981) and by Östman and Kjellin (2002) indicated that gender is regularly, however not indisputably, a factor that can affect, produce, or sustain stigmatisation. In chapter 3 , the effect of a family member's gender on his or her experiences of stigma by association was explored. In the study presented in chapter 3 , mothers and wives, more than fathers and husbands, of people with mental illness reported being accused of overprotectiveness, something that was perceived to contribute to the onset and continuation of their family member's mental illness. They also reported that such accusations increased psychological distress and diminished quality of life. Potentially, this is because mothers and wives tend to be especially caring and nurturing towards their family member with mental illness. This is in line with the social role theory (Eagly, 1987; Eagly \& Crowley, 1986; Eagly et al., 2000; Eagly \& Wood, 2012). The social role theory posits that gender roles are socially defined and reflect the historical division between men, who often have breadwinner roles and take on responsibilities outside the home, and women, who often take on responsibilities at home and, increasingly, combine these responsibilities with work outside the home (Eagly, 1987; Eagly et al., 2000; Wood \& Eagly, 2012). According to the social role theory, expectations of men and women are transmitted from generation to generation, diverge over time, and, in time, they come to represent gender stereotypes that exert a strong influence over the social behaviour of men and women (Eagly, 1987; Eagly et al., 2000). Consequently, mothers and wives of people with mental illness are most likely seen as, or assumed to be, more caring but also more overprotective and patronising than male family members of people with mental illness and that perceived overprotectiveness on the part of mothers and wives may lend itself to blaming, resulting from the perception that these women are, in some way, responsible for the onset and continuation of their family member's mental illness. These findings are in line with findings presented by Wirth and Bodenhausen (2009), who found that gender typical cases of mental illness, symptoms, or behaviour evoke more negative affect, less sympathy, and less support. They also found that gender-typical behaviour that is attributed to personal traits results in that person being held personally responsible for the stigmatised condition (Weiner, 1995; Wirth \& Bodenhausen, 2009). 


\section{Heredity}

In 2002, Phelan reported an increased emphasis on the biological causes and hereditary nature of mental illnesses in society. Family members of people with mental illness and researchers have expressed hopes that the perceived heredity of mental illnesses will function to reduce stigma (Mehta \& Farina, 1997), while others fear that an increased belief that mental illness is hereditary will increase stigma (Phelan, 2002).

As reported in chapters 2 and 3, family members who believed that mental illnesses are hereditary also reported more stigmatisation by association and psychological distress. Logically, they also tended to not hold their family member with mental illness responsible for their condition and thus experienced any stigmatisation towards that person or stigma by association towards themselves to be a gross societal injustice. This may have made them more alert to and sensitive about behaviour that can be construed as stigmatising.

Among some family members of people with mental illness, particularly children of people with mental illness, pregnant family members within the immediate family of a person with mental illness, and among parents of people with mental illness who have children of child-bearing age, a fear of genetic contamination and psychological distress was reported.

\section{Stigma by association and family burden}

Few studies have examined the relationship between stigma by association and family burden among family members of people with mental illness. The few studies that do exist differ in the extent to which they see stigma by association and family burden as separate constructs. Some of these studies claim that stigma by association is a contributing factor to feeling burdened (Chang \& Horrocks, 2006; Lefley, 1989; Marshall, 2013) and some specifically claim that stigma by association is a form of psychosocial or subjective burden (De Boer et al., 2008; Sales, 2003). Others, however, claim that stigma by association predicts family burden (Werner et al., 2012). In fact, Werner and colleagues (2012) contend that stigma by association likely impacts objective rather than subjective burden. Still others, like Mak and Cheung (2012) claim that stigma by association and burden are separate constructs.

Corrigan and Miller (2004) believe that the impact of stigma by association and family burden should be distinguished from one another, and that future research should also distinguish between the impact of family burden and the impact of stigma by association. They claim that this is the first step towards developing adequate stigma reduction strategies (Corrigan \& Miller, 2004). Consequently, the studies presented in chapters 4 and 5 explored, firstly, the relationships between family members' experiences of 
stigma by association, psychological distress, perceived closeness, and quality of life, and, secondly, the relationships between family burden, psychological distress, perceived closeness, and quality of life. However, as previously noted by Östman and Kjellin (2002) and by Lefley (1989), the participants in the qualitative study presented in chapter 4 found it difficult to differentiate between stigma by association and family burden in terms of both their experiences and the consequences of both. Their experiences of stigma by association and family burden and the consequences of stigma by association and family burden overlapped. In the quantitative study presented in chapter 5 , however, stigma by association and family burden both accounted for a largely unique variance in the outcomes of psychological distress and quality of life. These findings suggest that, although stigma by association is associated with family burden, and their consequences overlap, the two constructs are not identical. They are, however, intertwined and likely affect one another.

\section{Coping with stigma by association and family burden}

\section{Coping Strategies}

Crocker et al. (1998) previously noted that stigmatised people do not passively accept the predicaments of their stigma; rather, people actively attempt to cope with these predicaments. Likewise, family members of people with mental illness employ various strategies to cope with their experiences and the negative impact of stigmatisation and family burden (Saunders, 2003; Major \& O'Brien, 2005). How family members of people with mental illness cope with their experiences of perceived stigma by association and family burden was investigated both qualitatively and quantitatively in chapters 4 and 5. In these studies, family members reported using problem-focused and emotionfocused coping strategies to mitigate their experiences of perceived stigma by association and family burden and the negative impact of stigma by association and family burden. This is in line with findings by Eaton et al. (2011) and Hatfield and Lefley (1993), both of whom showed that family members of people with mental illness use emotionfocused coping strategies and problem-focused coping strategies to prevent, target, or reduce the frequency, intensity, and consequences of stigma by association and family burden.

The problem-focused coping strategies used by family members of people with mental illness reported in the qualitative study presented in chapter 4 mainly aimed at obtaining social, instrumental, or emotional support. Selective disclosure, organising support systems, seeking empathy, talking to professionals, and asking for professional support were frequently reported as problem-focused coping strategies used by family members of people with mental illness. Organising support systems and selective disclosure, in particular, were perceived as important ways of coping as they are thought to increase understanding and support, and prevent stigmatisation by association and fami- 
ly burden in the long run. These findings are similar to Bos et al. (2009), who found that selective disclosure is frequently used by mental health services users as a way of generating social support and diminishing stigmatisation.

Most of the emotion-focused coping strategies reported in chapter 4 seemed to be aimed at evading the stressor, or distracting or distancing oneself from the stigmatised condition or person. Distraction, substance use, disengagement or withdrawal from the family member with mental illness or the stigmatised situation, venting, and denial were regularly reported as emotion-focused coping strategies family members used to realise a mostly short-term reduction in negative emotions resulting from stigma by association and family burden. Acceptance, however, was associated with long-term improvement. Acceptance may simply make family members reappraise the stigmatised condition, make the stigmatised condition appear less negative, or stimulate the continuation or resumption of problem-focused coping actions. The findings of the qualitative study in chapter 4 are similar to those of Clarke and Tanya (2009), who found most emotion-focused coping strategies to be associated with short-term stress reduction and improvements in one's quality of life and problem-focused coping strategies to be associated with long-term improvement.

In the quantitative study reported in chapter 5 , the mediation effects of fourteen different coping strategies (i.e., five problem-focused coping strategies and nine emotionfocused coping strategies) on the relationships between stigma by association, family burden, psychological distress, and quality of life were examined. The problem-focused coping strategies principally had no mediation effect on these relationships, with the exception of seeking emotional support, which had competitive mediation effects, meaning that the use of this strategy was associated with less psychological distress and greater quality of life. In the relationship between family burden and quality of life, however, almost all problem-focused coping strategies had competitive mediation effects. Emotion-focused coping strategies, on the other hand, were predominantly associated with complementary mediation effects on these relationships (i.e., they may have made things worse); only positive reframing and acceptance had competitive mediation effects. Accepting that a family member has a mental illness, or reframing the family member's mental health condition more positively, may therefore be a precursor to providing support to these family members.

The findings in chapters 4 and 5 are similar to those reported by Aspinwall and Taylor (1992), who, in their longitudinal study of stress and psychological well-being among academic freshmen, found that the non-use of avoidance and the greater use of active coping strategies such as seeking social support mediated the beneficial effects of control, optimism, and self-esteem on one's adjustment to the stressful situation or condition. The findings are also consistent with Eaton et al.'s (2011) study among family 
members of people with mental illness in which the coping strategies found to be used most frequently by family members were acceptance of the stigmatised situation, seeking support, passive appraisal as a form of cognitive distraction, avoidance, and spirituality. Family members of people with mental illness also utilised resources and support systems such as mental health professionals, immediate family members, and their churches (Eaton et al., 2011). Crocker et al. (1998) noted that stigmatised people might deal with stigma through psychological disengagement or by withdrawing from an ingroup. Stigmatised people might therefore psychologically disengage their selfevaluation and self-esteem from their outcomes in particular stigma-threatening domains, contexts, or situations, especially when they have been the target of prejudice, have been threatened by stereotyping, or when the nature of the stigma makes success unlikely (Crocker et al., 1998).

The findings of both the qualitative study in chapter 4 and quantitative study in chapter 5 suggest that family members of people with mental illness are likely to take action or engage in problem-focused coping strategies when their quality of life is jeopardised by experiences of stigma by association or family burden. In addition, organising support systems, selective disclosure, and seeking emotional support from other family members, community members, and professionals appeared to be particularly significant mediators in mitigating the negative impact from stigma by association and family burden. These findings are in line with those of Miller and Major (2000), who claim that people mitigate the negative consequences of their stigma-related problems by using problem-focused coping strategies in an attempt to alter their relationship with their environment. Additionally, Miller and Major (2000) claim that emotion-focused coping strategies are used to regulate negative emotions from stigma-related stressors. The results presented in chapters 4 and 5 of this dissertation also suggest that most emotion-focused coping strategies seek to avoid, evade, or deny the stigmatised condition or distract or distance oneself from the stressor, and thus diminish well-being in the long run. However, acceptance and positive reframing, both of which are also emotionfocused coping strategies, appear to lead to a reappraisal of the stigmatised condition, stimulate the onset or continuation of problem-focused coping strategies, and are often precursors to providing social, emotional, or financial support to family members with mental illness. The findings in chapters 4 and 5 also support those of Lazarus (1993) who claimed that there is a strong tendency in Western societies to venerate problem-focused coping strategies and distrust emotion-focused coping strategies. Engaging problems and taking action against these problems, as most problem-focused coping strategies do, tend to be valued more than reappraising the relational meaning, as most emotion-focused coping strategies do. Nevertheless, there are sufficient indications that under certain conditions (e.g., when nothing useful can be done to change the situation), problem-focused coping strategies can be counterproductive (Carver \& 
Schreier, 1994; Sugiura, 2006); then, emotion-focused coping strategies may be best coping choice (Lazarus, 1993).

In looking at the findings reported in chapters 4 and 5, it is interesting to note that although the findings of the quantitative study in chapter 5 support some of those yielded from the qualitative study reported in chapter 4 , they are in conflict with others. In both studies, seeking emotional support appeared to prevent or lessen family members' experiences of stigma by association and family burden. Emotional support was also found to mitigate the negative impact of stigma by association and family burden in both studies. The qualitative study presented in chapter 4 suggests that not only seeking emotional support but also other problem-focused coping strategies may mediate the relationships between stigma by association and psychological distress and between family burden and psychological distress, while the quantitative study presented in chapter 5 showed limited evidence for such mediating effects. One must therefore question why the findings as they pertain to stigma by association, family burden, and their negative impact differ between the qualitative and quantitative studies in chapters 4 and 5 . One explanation is that the differences are rooted in the methodology (Onwuegbuzie, \& Teddlie, 2003). Qualitative research, and especially the use of in-depth interviews, has the potential to expose the nuances of how people react to complex social experiences, but it also relies upon and is therefore limited to participants' self-understanding of the various constructs and relationships within that study. In quantitative research, constructs are operationalised by, embedded in, and restricted to specific predefined questions, which probably limit participants' interpretation and self-understanding of these constructs (Carr, 1994). Because the constructs stigma by association and family burden were defined and operationalised more precisely in the quantitative study, family members of people with mental illness may have been better able to distinguish between the consequences of stigma by association and those of family burden.

\section{Adaptive versus maladaptive coping strategies?}

Another possible explanation for the differences between the effects of the various coping strategies may lie in the adaptiveness of coping strategies (Kohn et al., 2003; Zeidner \& Saklofske, 1996). Coping strategies are often appraised as predominantly adaptive or maladaptive (Moore et al., 2011). Adaptive coping strategies are assumed to mitigate psychological distress and improve quality of life. Maladaptive coping strategies, in contrast, may only reduce symptoms while simultaneously maintaining or even strengthening the stressor. Thus, although maladaptive coping strategies may temporarily mitigate the negative impact of stigma by association and family burden, they seem to be counterproductive and ineffective in the long run (Moore et al., 2011). 
Some of the coping strategies discussed in the studies presented in chapters 4 and 5 , such as self-distraction, denial, substance use, behavioural disengagement, and venting, likely have a negative effect on family members' adaptability to stigmatised conditions or situations as their aim is to escape or avoid feelings of psychological distress, and indeed, the findings of these studies suggest that avoiding, escaping, or ignoring the stigmatised condition or person may lead to inactivity or detachment and may subsequently generate feelings of hopelessness. These findings are similar to work conducted by Blum and Silver (2008) and Pulla et al. (2013). Blum and Silver (2008) found that the nature of a stressor sometimes overwhelms one's coping resources and, when this occurs, people may disengage from the stressful situation altogether using most often maladaptive coping strategies (e.g., substance use, denial, withdrawal, venting, disengagement) rather than engaging the stressful situation through adaptive coping strategies. Blum and Silver (2008) also noted that people often find comfort in disengagement from reality but only for a short time. In the end, the stressful situation tends to remain unaltered and inevitably resurfaces. Examples of maladaptive coping strategies, according to Pulla et al. (2013), include dissociation, distraction, disengagement, and avoidance and escape behaviours including substance use. Pulla et al. (2012) also found that maladaptive coping strategies may provide some relief through an immediate symptomatic reduction but that the stressful situation maintains and contributes to the growth of the stressor.

Maladaptive coping strategies (e.g., substance use, denial, distraction, behavioural disengagement, venting, and self-blame) thus appear to maintain the negative impact of stigma by association in the long run while adaptive coping strategies like the use of emotional support, the use of instrumental support, planning, positive reframing, and acceptance, tend to have a positive effect on family members' adaptability to the stigmatised condition when employed proactively, as they actually combat and alter the stressor and its consequences. They thus are likely to improve family members' personal situation and life satisfaction and may make family members conscious of opportunities to gain more control over the stigmatised condition and thereby actively improve their personal and family situation.

\section{Practical implications}

The studies in this dissertation contribute substantially to the theoretical understanding of stigma by association and add to the limited literature on stigma by association among family members of people with mental illness but an improved theoretical understanding is insufficient. It is also important to consider how the research findings can actually contribute to a reduction in stigma by association experiences and combat its negative impact. 
Corrigan and Miller (2004) have previously questioned whether changes in public stigma in the context of mental illness can result in decreased experiences of stigma by association and combat its negative impact. In the studies presented in chapters 2 and 3 , public stigma was found to be positively associated with stigma by association, and thus appears to be, at least partly, a preceding and related process to stigma by association (Angermeyer et al., 2003; Goffman, 1963; Mehta \& Farina, 1988; Neuberg et al., 1994; Pryor et al., 2012). As the findings demonstrate, family members of people with mental illness appear to suffer as a result of expressions of public stigma towards their family member with mental illness (i.e., vicarious stigma; Corrigan \& Miller, 2004). Consequently, interventions and stigma reduction strategies geared to both stigma by association and public stigma are necessary.

In their paper on the use of stigma reduction strategies to combat HIV- related stigma, Bos et al. (2008) outlined that, to increase the effectiveness of stigma reduction interventions and programs, these interventions and programs must not only provide information but also build skills and create opportunities for personal contact between the general public and people with the stigmatised condition. Likewise, Corrigan, Morris, Michaels, Rafacz, and Rüsch (2012) and Corrigan and Kosyluk (2013) identified three general strategies to reduce the stigmatisation of people with mental illness and their family members, namely education, contact, and protest. Corrigan and colleagues (2004; 2013) have also claimed that contact and education are promising approaches for the improvement of positive behavioural intentions and attitudes towards people with mental illness and their family members and Corrigan and Kosyluk (2013) demonstrated that face-to-face contacts have more impact than media-based approaches. Positive interactions between, on the one hand, people with mental illness and their family members, and, the general public, on the other should, therefore, be promoted and supported. In this context, positive "storytelling" by people with mental illness and their family members is likely to have a significant impact in changing intentions and attitudes towards people with mental illness and their family members.

Stigma reduction interventions, however, should not only focus on community members in general, but also on members of 'power groups' (e.g., politicians, employers, civil servants, and health care providers: Corrigan \& Miller, 2004) because they are important decision makers on subjects that directly impact the life goals and opportunities of people with mental illness and their family members. Power group members should therefore be informed and educated on the underlying processes, misconceptions, and negative impact of public stigma and stigma by association. Particularly health care providers, civil servants, and employers as power group members should also be offered skill building training so that they can better support people with mental illness and their family members. Power group members should also be invited to meet and interact with people with mental illness and their family members in order to 
acquire and enlarge positive interactions as well. Moreover, people in key power positions should be encouraged to enhance the life opportunities of people with mental illness and their family members by, for example, supporting people with mental illness and their family members, disclosing personal experiences with mental illness or people with mental illness, and by combating public stigma, stigma by association, and structural stigma in society at large as well as in workplaces, schools, clubs, and their local community.

The findings presented in this dissertation also suggest that, in order to mitigate the experiences and negative consequences of stigma by association (i.e., increased psychological distress, reduced perceived closeness, and diminished quality of life) among family members of people with mental illness, various coping strategies can be used. By learning more about how families and individual family members cope with their experiences and consequences of stigma by association, mental health professionals and experts will be able to suggest and encourage the use of advantageous coping strategies that support family functioning and improve the functioning of individual family members. This is particularly important because, at the onset of a family member's mental illness, most families have limited knowledge about the mental health condition and its implications. As such, families should be provided with not only information on the condition, they should also receive information on the psycho-social implications of the condition and be provided with advice on how to best cope with these implications. Mental health professionals and experts should therefore be trained to offer information, education, and support on the use of coping strategies so that they can help family members enhance existing advantageous coping skills and facilitate the development of new advantageous coping skills (Phelan et al., 2002; Shah et al., 2010). In the implementation of this, it is important to note and take into account that the effectiveness of any given coping strategy is not universal but dependent on the mental health condition, the family context, and one's personal characteristics as well.

Thus far, a number of recommendations that include mental health professionals have been made. It is, however, important to note that a number of participants in the study presented in chapter 3 reported a lack of interest on the part of mental health professionals. They often felt that their perspectives on care, familial relationship, family responsibility, and their problems and concerns were not taken seriously. Exclusion from, or non-participatory, treatment plans were perceived as very stigmatising by family members of people with mental illness and this kind of action apparent to have a seriously detrimental effect on the support, self-esteem, and psychological well-being of family members of people with mental illness. If mental health professionals and civil servants are to overcome the perception that they stigmatise family members of people with mental illness and show a lack of interest in the burden carried by family members, they must pay specific attention to, and demonstrate a personal interest in, 
stigma by association and its negative impact, especially if mental health professionals and civil servants want to optimally meet the needs of people with mental illness and their families. Mental health professionals should, therefore, continue to study the consequences that mental illnesses and stigma have for not only the person with a mental health condition but also their family in general and individual family members in particular. Moreover, anti-stigma programs should be crafted to challenge and change the prejudices and discriminatory actions of health professionals and civil servants. In such programs, family members of people with mental illness should be involved in all phases from design to implementation and evaluation (Bos et al., 2008).

Because most parents and spouses take on an explicit caregiving and supportive role in order to help their family member with mental illness, they need not only (psycho)education but also possibilities for respite from care (i.e., creating a caregiving free sphere or getting out of the caregiver world; Strang, Haughey, Gerdner, \& Teel, 1999). Creating caregiver respite can include offering access to a (mental health) day centre, offering the use of respite care services so that family members can rest or take a vacation, and creating opportunities for family members to engage in social and personal activities. Children and siblings of people with mental illness also appear to need periods of respite (Goossens \& Van der Zanden, 2012) and thus such facilities should be made available to these family members as well. Additionally, given that social support systems appear to be crucial for the enablement of periods of respite, it is important that family and social support systems for family members of people with mental illness be organised and/or maintained.

Siblings and children of people with mental illness - mostly those who had young children, were pregnant, or had a desire to have children - often reported worrying about the possibility that mental illness is hereditary and that their (potential) children could be 'genetically contaminated' by their family member with mental illness. This finding is similar to findings reported by Stålberg, Ekerwald, and Hultman (2004) who found that siblings of people with schizophrenia worry about passing on their "bad genes" to their children and therefore think that they are responsible for their child's mental illness, should mental illness present. In the studies presented in this dissertation, some parents of (adult) children with mental illness also feared that mental illness would be passed on to their (potential) grandchildren as well. Interestingly, parents, siblings, and children of people with mental illness appeared to not only be wary of possible genetic contamination and the subsequent stigmatisation their (potential) (grand)children could be subjected to, they also had concerns about experiencing vicarious stigma (i.e., feeling a sense of helplessness and sadness when one observes a family member being the object of discrimination and prejudice because of his or her mental illness; Corrigan, Druss, \& Perlick, 2014), and reliving their previous experiences of stigma by association. Taking this into account, it is possible that family members of people with mental illness 
have an increased need for information on the extent to which a mental illness is hereditary. Information and education on these topics should, therefore, be offered to family members of people with mental illness.

Siblings of people with mental illness, especially in their puberty or adolescence, need special attention from parents and mental health professionals. Adults should encourage siblings to attend psycho-educational, on-line group discussions, or events offered by self-help groups (Seeman, 2013), but only on a strictly voluntarily basis. Above all, parents should refrain from directing all parental attention and care to the child with mental illness. They must remain aware of the needs of their other children, try to involve siblings in treatment programs on their own terms, and prevent sibling rivalry (i.e., jealousy, fighting, and competition between siblings; University of Michigan Health System, 2009). The Development and Behavior Resources at the University of Michigan Health System (University of Michigan Health System, 2009) and the Sibling Support Project (Sibling Support Project, n.d.) offer relevant literature and education programs for siblings of people with special needs. Family members and mental health professionals may find this literature and these education programs to be useful when dealing with the impact of sibling rivalry and in balancing the needs of the child with mental illness and the needs of his or her siblings. Access to information about these programs and the literature is available at www.med.umich.edu.

\section{Methodological strengths and limitations}

This section briefly discusses the methods employed to explore the processes, experiences, and consequences of stigma by association in the studies reported in this dissertation.

One of the most important strengths of this dissertation is the use of quantitative as well as qualitative research methods. This mixed methods approach leans on the interplay between deductive and inductive reasoning and combines the advantages of large scale data sets amenable to statistical inferences with the rich detail of complex phenomena, detailed insight in experiences of individual participants, and identification of relevant contextual factors (Creswell, 2003; Teddlie \& Tashakkori, 2009). Qualitative research is often criticised as biased, lacking rigour, and being small scale. However, in the qualitative studies reported in this dissertation (chapters 3 and 4), research quality and rigour was optimised by using protocolled, semi-structured interviews. Generalisation was also supported by counting events and applying quasi-statistics (Litwin, 1995; Maxwell, 2010; Strauss \& Corbin, 1996). Additionally, a specific computer program for qualitative research (i.e., Nvivo) was used to increase the likelihood that data would be analysed systematically and data was processed anonymously as promised to participants (Seale \& Silverman, 1997). 
Another strength of this dissertation is the use of extended and advanced multiple mediation analyses and bootstrapping techniques in chapter 5 (Preacher \& Hayes, 2004). Bootstrapping techniques can increase statistical power and the mediation techniques put forth by Preacher and Hayes (2004) are believed to provide better information than statistical significance tests that assume normality of distribution across samples (e.g., the causal steps approach by Baron and Kenny, 1986; Hayes, Slater, \& Snyder, 2008). When Baron and Kenny's (1986) approach is used, researchers are not always able to distinguish between a mediation effect and an indirect effect, and they may, therefore, prematurely end their search for indirect effects if evidence that an independent and outcome variable are associated is absent. Furthermore, Preacher and Hayes' (2004) mediation techniques allow researchers to pit various theories against one another in a single model, while eliminating the problem of estimation bias, which can occur when multiple intercorrelated mediators are examined individually as is the case in Baron and Kenny's (1986) mediation model (Hayes, 2009). In essence, the use of both bootstrapping and advanced multiple mediation techniques employed in this dissertation likely enlarged the statistical power and reliability of the quantitative study presented in chapter 5 of this dissertation.

Despite strengths, the studies in this dissertation are also subject to some limitations. One is that, given the cross-sectional nature of the quantitative data, no conclusions regarding causality can definitively be drawn. Additionally, the reliance on self-reported methods could be a possible limitation as self-report methods are open to selfpresentational bias (Donaldson \& Grant-Vallone, 2002; Guglielmi, 1999). However, efforts were made to reduce this bias by using previously validated and frequently used measures and scales in the quantitative study and by assuring participants in the both qualitative and quantitative studies that their data would be processed anonymously. The use of retrospective reports may also be a limitation as this could generate recall bias. Recall bias, however, was combatted in the qualitative studies by asking follow-up questions in an effort to attain clear and detailed information in participants' descriptions of their experiences of stigma by association and family burden. An additional bias potentially present in the results is selection bias as the recruitment of participants in the qualitative studies relied heavily on support groups. To tackle selection bias, participants were recruited through multiple self-help support groups, professionally operated support groups, and mental health organisations with relevant, but differentiated, goals and group members. Clearly, these limitations have the potential to limit the generalisability of the findings presented in this dissertation. However, it is important to acknowledge that the qualitative studies presented in this dissertation did not necessarily seek to attain generalisability. At the same time, findings derived from qualitative research can often be transferable and thus invite readers to make connections 
between elements illuminated by the qualitative studies and their own experiences (Lincoln \& Guba, 1985).

Lastly, it is important to consider the fact that the findings of the quantitative study in chapter 5 support some of the predictions derived from the qualitative study in chapter 4 but conflict with others. Possibly, these findings and outcomes differ due to the different strengths and weaknesses of quantitative and qualitative research methods (e.g., the specificity and precise definition of constructs and variables within the research). Qualitative research provides depth and detailed insight concerning participants' attitudes, feelings, and behaviours, and allows participants to expand upon their responses. This can generate new themes that have not previously been considered. However, qualitative research relies upon participants' self-understanding of the relationships examined, making systematic comparisons and delineations difficult. Quantitative research, in contrast, provides numerical and larger scale data with statistical power, and uses more precisely defined constructs and variables. This allows for more precise measurement but, at the same time, through its focus on testing theories and hypotheses, it limits the scope of investigation and may fail to assess or bring to light relevant phenomena (e.g., participants' essential attitudes, feelings, and behaviours; Onwuegbuzie, \& Teddlie, 2003). It is possible that, because the constructs of stigma by association and family burden were more precisely operationalised in the quantitative studies than in the qualitative studies, family members of people with mental illness were better able to distinguish between the consequences of stigma by association and those of family burden in the quantitative study. Despite the fact that the use of qualitative and quantitative methods did not generate identical findings, the use of mixed methods in this dissertation is considered to be a methodological strength. Qualitative and quantitative research methods have different strengths and weaknesses; one approach is not superior to the other, and, ideally, both should be applied in combination (Carr, 1994).

\section{Recommendations for future research}

The studies in this dissertation have contributed to an increased theoretical understanding of the underlying processes, experiences, and consequences of stigma by association and family burden among family members of people with mental illness. They have also advanced our knowledge about the impact of a number of strategies that can be employed to cope with stigma by association and family burden, and provided guidelines for the reduction and prevention of stigma by association and its impact. Despite this, the current body of literature on stigma by association among family members of people with mental illness remains limited. More research on the processes underlying stigma by association and the ways in which its negative impact can be mitigated is needed. 
In the studies presented in chapters 2 and 3, experiences of public stigma were found to be positively associated with experiences of stigma by association. This finding was similar to results found by Pryor et al. (2012), whereby public stigma was related to stigma by association and spread from the stigmatised person to previously nonstigmatised persons. That study also showed that family associations represented an important basis for stigma by association. Those results and the results presented in chapters 2 and 3 of this dissertation suggest that future research should examine, in more detail than has been the case in this dissertation, how the preceding process of public stigma is associated with experiences of stigma by association among family members of people with mental illness. Specific attention should be given to exploring the degree to which public mental health stigma reduction strategies reduce stigma by association among family members of people with mental illness. It is important to further investigate this relationship, unravel its underlying processes and associations, and examine the strategies that may mitigate or prevent public stigma and stigma by association. Accordingly, evaluations appraising the effectiveness of mental health stigma reduction interventions should examine their effects on both public stigma and stigma by association.

Gender differences and other relevant characteristics such as familial relationship, coresidence, and the belief that mental illness is hereditary are also known to have effects on stigma, stigma by association, and the consequences of stigma by association (Corrigan \& Watson, 2007; Farina, 1981; Lanquetot, 1984; Phelan, 2002; Read \& Harré, 2001; Swets, Middeldorp, \& Schoevers, 2009; Wirth \& Bodenhausen, 2009) and the findings of the studies presented in chapters 2 and 3 do indeed show that family member characteristics have a diverse and significant impact on the experiences and consequences of stigma by association. It is therefore important to take these characteristics into account in future studies of stigma.

Also, given that experiences of stigma by association have a long and enduring influence on family members' lives and personal development (Corrigan \& Miller, 2004; Lanquetot, 1984; Ramchandani \& Stein, 2003; Robinson et al., 2008), more insight concerning the possible impact of stigma by association on the lives and individual development of young family members of people with mental illness is recommended as young family members of people with mental illness may have specific experiences of stigma by association. Ideally, a longitudinal design would be adopted to investigate the experiences and consequences of stigma by association among young family members of people with mental illness in order to examine changes over time and in order to determine the impact of stigma by association in the long run. Robinson et al. (2008) previously found, among children that are dependent on their parents, that having a parent with mental illness negatively affects the child's socio-emotional well-being, detrimentally impacts the parental care provided, and places these children at in- 
creased risk for developing mental health problems themselves. Children may also grieve the loss of their former relationship with their parent and thus display a range of feelings and reactions such as confusion, anger, and fear. It is therefore important that future stigma research, and particularly longitudinal stigma research, include family members of people with mental illness under the age of 18 . Such research should also explicitly investigate the effect of problem-focused and emotion-focused coping strategies on the relationships between stigma by association, psychological distress, perceived closeness, and quality of life among young family members of people with mental illness.

Given that people with mental illness and their family members not only experience stigma and stigma by association from the general public, but also from people close to them such as other family members, future research should investigate processes of stigmatisation within familial dyads of people with mental illness and, in doing so, pay particular attention to the effect of stigma by association on these processes. Some previous research has been done in this regard. For example, Wight, Aneshensel, Murphy, Miller-Martinez, and Beals (2006) studied dyads of people with HIV and their caregiving family members and found that stigma by association may have negative consequences for the dyadic relationship. More specifically, they outline how becoming, by proxy, a member of a stigmatised community complicates the relationship between caregiving family members and the person with HIV and show how experiences of stigma by association can change the caregiver's perspective on their family member with HIV, evoking feelings of anger and regret. This study further showed that, simultaneously, the person with HIV can interpret their family members' changed perspectives, emotions, and attitudes as stigmatisation, making them feel blamed and guilty. These changed perspectives, emotions, and attitudes may also elicit processes of mutual stigmatisation. It is quite plausible that, also with mental illness, processes of public stigma, stigma by association, and dyadic stigma are similarly intertwined. Consequently, it is important that future research explore the possible unique influence mutual stigmatisation and dyadic stigma may have on the caregiving dyad in order to allocate resources aimed at ameliorating stigma within familial dyads impacted by mental illness.

Lastly, the studies presented in chapters 4 and 5 underlined the similarities and differences between stigma by association and family burden. Earlier research by Östman and Kjellin (2002) and by Lefley (1989) reported that participants found it difficult to differentiate between their experiences of stigma by association and family burden. Various researchers have categorised stigma by association as a subjective burden (De Boer et al., 2008; Sales, 2003), while others have defined stigma by association as a determinant of objective rather than subjective burden (Werner et al., 2012). Still others view stigma by association and family burden as two separate constructs (Mak \& 
Cheung, 2012). Given that the findings reported in chapters 4 and 5 also lack concurrency on this aspect, a final recommendation is that future studies further investigate the relationship between stigma by association and the different dimensions of family burden.

\section{Conclusion}

This chapter summarised the findings of the studies reported in this dissertation and discussed their contribution to the field of stigma by association. Using both quantitative as well as qualitative research methods, these studies explored aspects of stigma by association and focused on family members of people with mental illness. These studies examined family members' experiences of stigma by association and its consequences, and investigated the strategies family members of people with mental illness use to cope with their experiences of stigma by association and its consequences. As such, this dissertation has contributed to an increased understanding of the social and psychological processes underlying stigma by association and has thereby contributed to the literature on stigma by association. Several starting points for future research have also been provided so that we can continue to disentangle the relationships between stigma by association, family burden, and the impact of stigma by association and family burden, as well as the coping strategies employed by family members of people with mental illness.

"People fear what they don't understand. And let's face it, mental health has only recently begun to even be an acceptable topic of conversation. Unfortunately, for many, it is still a topic that sends shivers down spines, but it doesn't have to stay that way. By simply talking about it, we normalize it. I have a feeling that, eventually, people will start to understand."

(Chris Curry, 2012) 

REFERENCES 

Adato, M. (2011). Combining Quantitative and Qualitative Methods for Program Monitoring and Evaluation: Why Are Mixed-Method Designs Best? (World Bank Other Operational Studies 11063). Retrieved July 20, 2014, from The World Bank at http://siteresources.worldbank.org/INTPOVERTY/Resources/3356421276521901256/premnoteME9.pdf.

Agnew, C. R., Loving, T. J., Le, B., \& Goodfriend, W. (2004). Thinking close: Measuring relational closeness as perceived self-other inclusion. In D. Mashek, \& A. Aron (Eds.), Handbook of closeness and intimacy (pp. 103-115). Mahwah, NJ: Erlbaum.

Aiken, L. S., \& West, S. G. (1991). Multiple regression: Testing and interpreting interactions. Newbury Park, CA: Sage.

Angermeyer, M. C., Liebelt, P., \& Matschinger, H. (2001). Befindlichkeitsstorungen der eltern von patienten mit schizophrenen oder affektiven storungen, psychotherapie, psychosomatic. Medizinische Psychologie 51(6), 255-260. doi:10.1055/s-2001-14299

Angermeyer, M. C., Schulze, B., \& Dietrich, S. (2003). Courtesy stigma: A focus group study of relatives of schizophrenia patients. Social Psychiatry Psychiatric Epidemiology, 38, 593-602. doi:10.1007/s00127-0030680-x

Aron, A., \& Aron, E. N. (1986). Love as the expansion of self: Understanding attraction and satisfaction. New York, NY: Hemisphere.

Aron, A., Aron, E. N., \& Smollan, D. (1992). Inclusion of Other in Self Scale and the structure of interpersonal closeness. Journal of Personality and Social Psychology, 63, 596-612. doi:10.1037/0022-3514.63.4.596

Aron, A., Aron, E. N., Tudor, M., \& Nelson, G. (1991). Close relationships as including other in the self. Journal of Personality and Social Psychology, 60, 241-253. doi:10.1037/0022-3514.60.2.241

Aspinwall, L. G. \& Taylor, S. E. (1992). Modeling Cognitive Adaptation: A Longitudinal Investigation of the Impact of Individual Differences and Coping on College Adjustment and Performance. Journal of Personality and Social Psychology, 63(6), 989-1003. doi: 10.1037//0022-3514.63.6.989

Banaji, M. R., Baron, A. S., Dunham, Y., \& Olson, K. (2008). The development of intergroup social cognition: Early emergence, implicit nature, and sensitivity to group status. In Levy, S. R. \& Killen, M. (Eds.), Intergroup attitudes and relations in childhood through adulthood (pp. 87-105). Oxford, England: Oxford University Press.

Baron, R. M., \& Kenny, D. A. (1986). The moderator-mediator variable distinction in social psychological research: Conceptual, strategic, and statistical considerations. Journal of Personality and Social Psychology, 51, 1173-1182. doi:10.1037/0022-3514.51.6.1173

Bathje, G. J., \& Pryor, J. B. (2011). The relationships of public and self-stigma to seeking mental health services. Journal of Mental Health Counseling, 33, 161-176.

Bazeley, P., \& Jackson, K. (2007). Qualitative data analysis with NVivo. London: Sage Publications Ltd.

Ben-Zeev, D., Young, M. A., \& Corrigan, P. W. (2010). DSM-V and the stigma of mental illness. Journal of Mental Health, 19(4), 318-27. doi:10.3109/09638237.2010.492484

Black, B., \& Miles, M. S. (2002). Calculating the risks and benefits of disclosure in African American women who have HIV. Journal of Obstetric, Gynaecologic and Neonatal Nursing, 31(6), 688-697. doi:10.1177/088421702129005335

Blum, S., \& Silver, R. C. (2008). Coping. In W. A. Darity, Jr. (Ed.), International encyclopedia of the social sciences, 2nd Edition (pp. 128-130). Detroit: Macmillan Reference. doi:10.1016/B978-0-12-375000$6.00110-5$

Bos, A. E. R. (2001). HIV stigma and social interaction: Examining strategies to influence perceivers' emotional and behavioral reactions in initial encounters Doctoral dissertation). Retrieved May 6, 2012, from http://dissertaties.ub.unimaas.nl/detail.aspx?id=10657

Bos, A. E. R., Kanner, D., Muris, P., Janssen, B., \& Mayer, B. (2009). Mental Illness Stigma and Disclosure: Consequences of Coming out of the Closet, Issues in Mental Health Nursing, 30, 509-513. doi:10.1080/01612840802601382

Bos, A. E. R., Kok, G., \& Dijker, A. J. (2001). Public reactions to people with HIV/AIDS in the Netherlands. AIDS Education and Prevention, 13, 219-228. doi:10.1521/aeap.13.3.219.19741 
Bos, A. E. R., Pryor, J. B., Reeder, G. D., \& Stutterheim, S. E. (2013). Stigma: Advances in Theory and Research, Basic and Applied Social Psychology, 35(1), 1-9. doi:10.1080/01973533.2012.746147

Bos, A. E. R., Schaalma, H. P., \& Pryor, J. B. (2008). Reducing AIDS-related stigma in developing countries: The importance of theory- and evidence based interventions. Psychology, Health \& Medicine, 13, 450-460. doi:10.1080/13548500701687171

Brohan, E., Slade, M., Clement, S., \& Thornicroft, G. (2010). Experiences of mental illness stigma, prejudice and discrimination: a review of measures, BMC Health Services Research, 10:80. doi:10.1186/1472-6963$10-80$

Burke, L. (2003). The impact of maternal depression on familial relationships. International Review of Psychiatry, 15, 243-255. doi:10.1080/ 0954026031000136866

Burnard, P. (2004). A method of analysing interview transcripts in qualitative research. Nurse Education Today, 11(6), 461-466. doi:10.1016/02606917(91)90009-Y

Burnard, P., Gill, P., Stewart, E., Treasure, K., \& Chadwick, B. (2008). Analysing and presenting qualitative data. British Dental Journal, 204, 429-432. doi:10.1038/sj.bdj.2008.292

Butter, A., Webster, M., \& Hill, M. (2010). Understanding the needs of people with mental health conditions and/or learning disabilities and the implications for the Pension, Disability and Carers Service (Research Report 654). Retrieved February 23, 2011, from https://www.gov.uk/government/uploads/system/uploads/attachment_data/file/214424/rrep654.pdf

Byrne, P. (2000). Stigma of mental illness and ways of diminishing it. Advances in Psychiatric Treatment, (6), 65-72. doi:10.1192/apt.6.1.65

Canam, C. (1993). Common adaptive forms facing parents of children with chronic conditions. Journal of Advanced Nursing, 18(1), 46-53.

Carr, L. T. (1994). The strengths and weaknesses of quantitative and qualitative research: what method for nursing? Journal of Advanced Nursing,20(4),716-721. doi: 10.1046/j.1365-2648.1994.20040716.x

Carver, C. S. (1997). You want to measure coping but your protocol's too long: Consider the brief COPE. International journal of Behavioral Medicine, 4, 92-100. doi:10.1207/s15327558ijbm0401_6

Carver, C. S., \& Scheier, M. F. (1994). Situational coping and coping disposition in a stressful transaction. Journal of Personality and Social Psychology, 66, 184-195. doi:10.1037//0022-3514.66.1.184

Carver, C. S., Scheier, M. F., \& Weintraub, J. K. (1989). Assessing coping strategies: A theoretically based approach. Journal of Personality and Social Psychology, 56(2), 267-283. doi:10.1037//0022-3514.56.2.267

Cassileth, B. R., Lusk, E. J., Strouse, T. B., Miller, D. S., Brown, L. L., Cross, P. A., \& Tenaglia, A. N. (1984). Psychosocial status in chronic illness. The New England Journal of Medicine, 311, 506-511. doi:10.1056/NEJM198408233110805

Chang, K. H. \& Horrocks, S. (2006). Lived experiences of family caregivers of mentally ill relatives. Journal of Advanced Nursing, 53(4), 435-443. doi:10.1111/j.1365-2648.2006.03732.x

Chou, K.L., \& Mak, K. (1998). Attitudes To Mental Patients Among Hong Kong Chinese: a Trend Study Over Two Years. International Journal of Social Psychiatry, 44(3), 215-224. doi:10.1177/002076409804400307

Chronister, J., Chan, F., Sasson-Gelman, J. \& Chiu, C. (2010). The association of stress-coping variables to quality of life among caregivers of individuals with traumatic brain injury. Neurorehabilitation, 27, 49-62.

Chronister, J., Chou, C. C., \& Liao, H. (2013). The role of stigma coping and social stigma in mediating the effect of societal stigma on internalized stigma, mental health recovery, and quality of life among people with serious mental illness. Journal of Community Psychology, 41(5), 582-600 doi:10.1002/jcop.21558

Clarke, D., \& Tanya, G. (2009). The mediating effects of coping strategies in the relationship between automatic negative thoughts and depression in a clinical sample of diabetes patients. Personality and Individual Differences, 46(4), 460-464. doi:10.1016/j.paid.2008.11.014

Conde-Sala, J. L., Garre-Olmo J., Turro-Garriga O., Vilalta-Franch J., \& Lopez-Pousa S. (2010). Differential features of burden between spouse and adult-child caregivers of patients with Alzheimer's disease: an exploratory comparative design. International Journal of Nursing Studies, 47(10), 1262-1273. doi:10.1016/j.ijnurstu.2010.03.001

Corrigan, P. W. (2005). On the Stigma of Mental Illness: Practical Strategies for Research and Social Change. Washington, D.C.: APA Books. 
Corrigan, P. W., Druss, B. G., \& Perlick, D. A. (2014). The impact of mental illness stigma on seeking and participating in mental health care. Psychological Science in the Public Interest, 15(2), 37-70. doi:10.117/15291000614531398

Corrigan, P. W., \& Kosyluk, K. A. (2013). Erasing the Stigma: Where Science Meets Advocacy. Basic and Applied Social Psychology, 35, 131-140. doi:10.1080/01973533.2012.746598

Corrigan, P. W., Larson, J. E., \& Kuwabara, S. A. (2007). Mental Illness Stigma and the Fundamental Components of Supported Employment. Rehabilitation Psychology 2007, 52(4), 451-457. doi:10.1037/00905550.52.4.451

Corrigan, P. W., Larson, J. E., \& Rüsch, N. (2009). Self-stigma and the "why try" effect: impact on life goals and evidence-based practices. World Psychiatry, 8(2), 75-81.

Corrigan, P., Markowitz, F., Watson, A. C., Rowan, D., \& Kubiak, M. A. (2003). An attribution model of public discrimination towards persons with mental illness. Journal of Health and Social Behavior, 44, $162-179$. doi:10.2307/1519806

Corrigan, P. W., \& Miller, F. E. (2004). Shame, blame, and contamination: A review of the impact of mental illness stigma on family members. Journal of Mental Health, 13(6), 537-548. doi:10.1080/09638230400017004

Corrigan, P. W., Morris, S. B., Michaels, P. J., Rafacz, J. D., \& Rüsch, N. (2012). Challenging the Public Stigma of Mental Illness: A Meta-Analysis of Outcome Studies. Psychiatric Services, 63(10), 963-973. doi:10.1176/appi.ps.201100529

Corrigan, P. W., \& Penn, D. L. (1999). Lessons from social psychology on discrediting psychiatric stigma. American psychologist, 54(9), 765-776. doi:10.1037//0003-066X.54.9.765

Corrigan, P.W. \& Shapiro, J. (2010). Measuring the impact of programs that challenge the public stigma of mental illness. Clinical Psychology Review, 30, 907-922. doi:10.1016/j.cpr.2010.06.004

Corrigan, P. W., \& Watson, A. C. (2002). Understanding the impact of stigma on people with mental illness. World Psychiatry, 1(1), 16-20.

Corrigan, P. W., \& Watson, A. C. (2007). The Stigma of Psychiatric Disorders and the Gender, Ethnicity, and Education of the Perceiver. Community Mental Health Journal, 43(5), 439-458. doi:10.1007/s10597-0079084-9

Corrigan, P. W., Watson, A. C., Byrne, P., \& Davis, K. E. (2005). Mental illness stigma: Problem of public health or social injustice? The Social Worker, 50, 363-368. doi:10.1093/sw/50.4.363

Corrigan, P. W., Watson, A. C., \& Miller, F. E. (2006). Shame, blame, and contamination: The impact of mental illness and drug dependence stigma on family members. Journal of Family Psychology, 20, 239-246. doi:10.1037/0893-3200.20.2.239

Crandall, C. S., \& Moriarty, D. (1995). Physical illness stigma and social rejection. British Journal of Social Psychology, 34, 67-83. doi:10.1111/j.2044-8309.1995.tb01049.x

Creswell, J. W. (2003). Research design Qualitative, Quantitative, and Mixed Methods Approaches. Thousand Oaks, CA: Sage Publications, Inc.

Crisp, A. H., Gelder, M. G., Rix, S., Meltzer, H. I., \& Rowlands, O. J. (2000). Stigmatisation of people with mental illnesses. British Journal of Psychiatry,177, 4-7. doi:10.1192/bjp.177.1.4

Crocker, J., Major, B., \& Steele, C. (1998). Social stigma. In D. Gilbert, S. T. Fiske, \& G. Lindzey (Eds.), The Handbook of Social Psychology (pp. 504-553). New York: McGraw Hill.

Crowe, A., \& Lyness K. P. (2013). Family Functioning, Coping, and Distress in Families With Serious Mental Illness. The Family Journal, 22(2), 186-197. doi:10.1177/1066480713513552

Curry, C. (2012, May 6). Mental Health Stigma: Prejudice and Discrimination [Web log comment]. Retrieved January 30, 2014, from http://www.healthyplace.com/blogs/survivingmentalhealthstigma/2012/05/prejudice-and-discrimination-in-mental-health-stigma/

De Boer, H. M., Mula, M., \& Sander, J. W. (2008). The global burden and stigma of epilepsy. Epilepsy and Behavior 12(4), 540-546. doi:10.1016/j.yebeh.2007.12.019

De Graaf, R., Ten Have, M., \& Van Dorsselaer, S. (2010). De psychische gezondheid van de Nederlandse bevolking, NEMESIS-2: Opzet en eerste resultaten. (Research Report Nemesis-2). Retrieved November 13, 2013, from http://www.trimbos.nl/ /media/af0898\%20nemesis\%20ii.ashx 
Dietrich, S., Beck, M., Bujantugs, B., Kenzine, D., Matschinger, H., \& Angermeyer, M. C. (2004). The relationship between public causal beliefs and social distance toward mentally ill people. Australian and New Zealand Journal of Psychiatry, 38, 348-354. doi:10.1080/j.1440- 1614.2004.01363.x

Dijker, A. J., \& Koomen, W. (2003). Extending Weiner's attribution-emotion model of stigmatisation of ill persons. Basic and Applied Social Psychology, 25(1), 51-68. doi:10.1207/S15324834BASP2501_4

Donaldson S. I., \& Grant-Vallone, E. J. (2002). Understanding self-report bias in organizational behaviour research. Journal of Business and Psychology, 17(2), 245-260.

Dovidio, J. F., Major, B., \& Crocker, J. (2000). Stigma: introduction and overview. In T. F. Heatherton, R. E. Kleck, M. R. Hebl, \& J. G. Hull (Eds.), The social psychology of stigma (pp. 1-29). New York: Guilford Press.

Drapalski, A. L., Lucksted, A., Perrin, P. B., Aakre, J. M., Brown, C. H., DeForge, B. R., \& Boyd, J. E. (2013). A Model of Internalized Stigma and Its Effects on People With Mental Illness. Psychiatric Services, 64(3), 264-269. doi:10.1176/appi.ps.001322012

Duffy, M. K., Ganster, D., \& Pagon, M. (2002). Social undermining in the workplace. Academy Of Management Journal, 45(2), 331-351. doi:10.2307/306935

Eagly, A. H. (1987). Sex differences in social behavior: A social-role interpretation. Hillsdale, NJ: Erlbaum

Eagly, A. H., \& Crowley, M. (1986). Gender and Helping Behavior: A Meta-analytic View of the Social Psychological Literature. Psychological Bulletin, 100, 283-308. doi:10.1037//0033-2909.100.3.283

Eagly, A. H., \& Wood, W. (2012). Social role theory. In P. van Lange, A. Kruglanski, \& E. T. Higgins (Eds.), Handbook of theories in social psychology (pp. 458-476). Thousand Oaks, CA: Sage Publications.

Eagly, A. H., Wood, W., \& Diekman, A. (2000). Social role theory of sex differences and similarities: A current appraisal. In T. Eckes \& H. M. Trautner (Eds.), The developmental social psychology of gender (pp. 123174). Mahwah, NJ: Lawrence Erlbaum.

Eaton, P. M., Davis, B. L., Hammond, P. V., Condon, E. H., \& McGee, Z. T. (2009). Coping Strategies of Family Members of Hospitalized Psychiatric Patients. Nursing Research and Practice, 2011, 1-12. doi:10.1155/2011/392705

Eldering, L. (2006). Cultuur en opvoeding. Rotterdam, the Netherlands: Uitgeverij Lemniscaat.

Ennis, E., \& Bunting, B. P. (2013). Family burden, family health and personal mental health. BMC Public Health, 13, 255. doi:10.1186/1471-2458-13-255

Ewertzon, M., Cronqvist, A., Lutzen, K., \& Andershed, B. (2012). A Lonely Life Journey Bordered with Struggle: Being a Sibling of an Individual with Psychosis. Issues in Mental Health Nursing, 33(3), 157-164. doi:10.3109/01612840.2011.633735

Farina, A. (1981). Are women nicer people than men? Sex and the stigma of mental disorders. Clinical Psychology Review, 1, 223-243. doi:org/10.1016/0272-7358(81)90005-2

Farina, A. (2000). The few gains and many losses for those stigmatized by psychiatric disorders. In J. H. Harvey \& E. D. Miller (Eds.), Loss and trauma: General and close relationship perspectives (pp. 183-207). Philadelphia, PA: Brunner-Routledge.

Farina, A., Fischer, J. D., \& Fischer, E. H. (1992). Societal Factors in the Problems Faced by Deinstitutionalized Psychiatric Patients, In P. J. Fink \& A. Tasman (Eds.), Stigma and mental illness (pp. 167-184). Washington, DC: American Psychiatric Press.

Feldman, D. B., \& Crandall, C. S. (2007). Dimensions of Mental Illness Stigma: What About Mental Illness Causes Social Rejection? Journal of Social and Clinical Psychology, 26(2), 137-154. doi:10.1521/jscp.2007.26.2.137

Fink, P. J., \& Tasman, A. T. (1992). Stigma and mental illness. Washington, DC: American Psychiatric Press.

Fiske, S. T. \& Taylor, S. E. (1991). Social cognition (2nd ed.). New York: McGraw-Hill.

Fortune, D. G., Smith, J. V., \& Garvey, K. (2005). Perceptions of psychosis, coping, appraisals, and psychological distress in the relatives of patients with schizophrenia: an exploration using self-regulation theory. British Journal of Clinical Psychology, 44(3), 319-331. doi:10.1348/014466505X29198

Gaebel, W., Bauman, A. E., \& Phil, M. A. (2003). Interventions to Reduce the Stigma Associated With Severe Mental Illness: Experiences From the Open the Doors Program in Germany. The Canadian Journal of Psychiatry, 48(10), 657-662. 
Galaif, E., Sussman, S., Chou, C. P., \& Wills, T. (2003). Longitudinal relations among depression, stress and coping in high risk youth. Journal of Youth and Adolescence, 32, 243-258.

Goffman, E. (1963). Stigma: Notes on the management of spoiled identity. Englewood Cliffs, NJ: Prentice-Hall.

González-Torres, M.A., Oraa, R., Arístegui, M., Fernández-Rivas, A., \& Guimon, J. (2007). Stigma and Discrimination towards People with Schizophrenia and their Family Members; a Qualitative Study with Focus Groups. Social Psychiatry and Psychiatric Epidemiology, 42, 14-23. doi:10.1007/s00127-006-0126-3

Goossens, F. X. \& Van der Zanden, A. P. (2012). Factsheet KOPP/KVO: Kinderen van ouders met psychische problemen, kinderen van verslaafde ouders (Trimbos-instituut Research Report AF1178). Retrieved December 8, 2014, from http://www.trimbos.nl/webwinkel/productoverzicht-webwinkel/psychischegezondheid/af/ /media/files/gratis\%20downloads/af1178\%20factsheet\%20kopp_kvo_web.ashx

Guglielmi, R. S. (1999). Psychophysiological assessment of prejudice: Past research, current status, and future directions. Personality and Social Psychology Review, 3, 123-157.

Hatfield, A. B., \& Lefley, H. P. (1993). Surviving mental illness: stress, coping, and adaption. New York: The Guilford Press.

Hayes, A. F. (2013). Introduction to mediation, moderation, and conditional process analysis. New York: The Guilford Press.

Hayes, A. F., Slater, M. D., \& Snyder, L. B. (2008). The Sage Sourcebook of Advanced Data Analysis Methods for Communication Research. Thousand Oaks, CA: Sage Publications, Inc.

Hebl, M. R., \& Mannix, L. M., (2003). The weight of obesity in evaluating others: A mere proximity effect. Personality and Social Psychology Bulletin, 29, 28-38. doi:10.1177/0146167202238369

Heubeck, B. G., \& Neill, J. T. (2000). Internal validity and reliability of the 30 item Mental Health Inventory for Australian Adolescents. Psychological Reports, 87, 431-440. doi:10.2466/PR0.87.6.431-440

Hoop, J. G. (2008). Ethical considerations in psychiatric genetics. Harvard Review of Psychiatry, 16, 322-338. doi:10.1080/10673220802576859

Jenkins, J. H., \& Schumacher, J. G. (1999). Family burden of schizophrenia and depressive illness: Specifying the effects of ethnicity, gender and social ecology. British Journal of Psychiatry, 174, 31-38. doi:10.1192/bjp.174.1.31

Johnstone, M. J. (2001). Stigma, social justice and the rights of the mentally ill: Challenging the status quo. Australian \& New Zealand Journal of Mental Health Nursery, 10, 200-209. doi:10.1046/j.14400979.2001.00212.x

Judge, K., (1994). Serving children, siblings and spouse: Understanding the needs of other family members. In H. P. Lefley \& M. Wasow (Eds.), Helping Families Cope with Mental Illness (pp.161-194). Newark, New Jersey: Harwood Academic.

Kessler, R. C., Berglund, P., Demler, O., Jin, R., Merikangas, K. R., \& Walters, E. E. (2005). Lifetime Prevalence and Age-of-Onset Distributions of DSM-IV Disorders in the National Comorbidity Survey Replication. Archives of General Psychiatry, 62, 593-602. doi:10.1001/archpsyc.62.6.593

Kessler R. C., Chiu W. T., Demler O., \& Walters E. E. (2005). Prevalence, severity, andcomorbidity of twelvemonth DSM-IV disorders in the National Comorbidity Survey Replication (NCS-R). Archives of General Psychiatry, 62(6), 617-627.

Kohn, P. M., O’Brien-Wood, C., Pickering, D. I., \& Decicco, T. L. (2003). The Personal Functioning Inventory: A Reliable and Valid Measure of Adaptiveness in Coping. Canadian Journal of Behavioural Science, 35(2), 111-123. doi: 10.1037/h0087193

Koschade, J. E., \& Lynd-Stevenson, R. M. (2011). The stigma of having a parent with mental illness: Genetic attributions and associative stigma. Australian Journal of Psychology, 63(2), 93-99. doi:10.1111/j.17429536.2011.00009.x

Kreisman, D. E., \& Joy, V. D. (1974). Family response to the mental illness of a family member: A review of the literature. Schizophrenia Bulletin, 1(10), 34-57. doi:10.1093/schbul/1.10.34

Kulik, C. T., Bainbridge, H. T., \& Cregan, C. (2008). 'Known by the company we keep: Stigma-by-association effects in the workplace'. Academy of Management Review, 33(1), 216-230. doi:10.5465/AMR.2008.27752765 
Kwekkeboom, H. M. (2000). De zorg blijft. Verslag van een onderzoek onder familieleden en andere relaties van mensen met (langdurige) psychische problemen. (Research Report No. 62). Retrieved March 28, 2013, from http://www.scp.nl/Publicaties/Alle_publicaties/Publicaties_2000/De_zorg_blijft

Lanquetot, R. (1984). First person account: Confessions of the daughter of a schizophrenic. Schizophrenia Buletin, 10(3), 467-471. doi:10.1093/schbul/10.3.467

Larson, E. J., \& Corrigan, P. W. (2008). The stigma of families with mental illness. Academic Psychiatry, 32(2), 87-91. doi:10.1176/appi.ap.32.2.87

Lazarus R. S., \& Folkman S. (1984). Stress, appraisal, and coping. New York: Springer.

Lefley, H. P. (1987a). Aging parents as caregivers of mentally ill adult children: An emerging social problem. Hospital \& Community Psychiatry, 38, 1063-1070.

Lefley, H. P. (1989). Family Burden and Family Stigma in Major Mental Illness, American Psychologist, 44(3), 556-560. doi:10.1037//0003-066X.44.3.556

Lewandowski, G. W., Aron, A., Bassis, S., \& Kunak, J. (2006). Losing a self-expanding relationship: Implications for the self-concept. Personal Relationships, 13(3), 317-331. doi:10.1111/j.1475-6811.2006.00120.x

Lincoln, Y. S., \& Guba, E.G. (1985). Naturalistic inquiry. Beverly Hills, CA: Sage.

Link, B. G., \& Cullen, F. T. (1990). The Labeling Theory of Mental Disorder: A Review of the Evidence. In Greenley, J. (Ed.), Mental Illness in Social Context (pp. 75-106). Greenwich, CT: JAI Press.

Link, B. G., Cullen, F. T., Struening, E. L., Shrout, P. E., \& Dohrenwend, B. P. (1989). A modified labelling theory approach to mental disorders: An empirical assessment. American Sociological Review, 54, 400-423. doi:10.2307/2095613

Link, B. G., Phelan, J. C., Bresnahan, M., Stueve, A., \& Pescosolido, B. A. (1999). Public conceptions of mental illness: Labels, causes, dangerousness, and social distance. American Journal of Public Health, 89(9), 1328-1333. doi:10.2105/AJPH.89.9.1328

Link, B. G., Struening, E. L., Rahav, M., Phelan, J. C., \& Nuttbrock, L. (1997). On stigma and its consequences: Evidence from a longitudinal study of men with dual diagnoses of mental illness and substance abuse. Journal of Health and Social Behavior, 38, 177-190. doi:10.2307/ 2955424

Litwin, M. S. (1995). How to Measure Survey Reliability and Validity. Thousand Oaks, CA: Sage Publications Inc.

Major, B., \& O’Brien, L. T. (2005). The social psychology of stigma. Annual Review of Psychology, 56, $393-421$. doi:10.1146/annurev.psych.56.091103.070137

Mak, W. W. S. \& Cheung, R. Y. M. (2012). Psychological Distress and Subjective Burden of Caregivers of People with Mental Illness: The Role of Affiliate Stigma and Face Concern. Community Mental Health Journal 48, 270-274. doi:10.1007/s10597-011-9422-9

Manne, S., Ostroff, J., Fox, K., Grana, G., \& Winkel, G. (2009). Cognitive and social processes predicting partner psychological adaptation to early stage breast cancer, British Journal of Health Psychology, 14(1), 4968. doi:10.1348/135910708X298458

Markowitz, F. E. (1998). The Effects of Stigma on the Psychological Well-Being and Life Satisfaction of Persons with Mental Illness. Journal of Health and Social Behavior, 39, 335-348. doi:10.2307/2676342

Marshall, O. (2013). Associative Stigma Among Families of Alcohol and Other Drug Users (master's thesis). Retrieved December 8, 2014, from http://ro.ecu.edu.au/theses_hons/104

Maxwell, J. A. (2010). Using Numbers in Qualitative Research. Qualitative inquiry,16(6), 475-482. doi:10.1177/1077800410364740

McCubbin, H. I., Nevin, R. S., Cauble, A. E., Larsen, A., Comeau, J. K., \& Patterson, J. M. (1982). Family coping with chronic illness: The case of cerebral palsy. In H. I. McCubbin, A. E. Cauble, \& J. M. Patterson (Eds.), Family stress, coping, and social support (pp. 169-188). Springfield, IL: Charles C Thomas.

Mehta, S. I., \& Farina, A. (1988). Associative stigma: Perceptions of the difficulties of college-aged children of stigmatised fathers. Journal of Social and Clinical Psychology, 7, 192-202. doi:10.1521/jscp.1988.7.23.192

Mehta, S., \& Farina, A. (1997). Is being "sick" really better? Effect of the disease view of mental disorder on stigma. Journal of Social and Clinical Psychology, 16(4), 405-419. doi:10.1521/jscp.1997.16.4.405 
Michaels, P. J., López, M., Rüsch, N., \& Corrigan, P. W. (2012). Constructs and concepts comprising the stigma of mental illness. Psychology, Society \& Education, 4(2), 183-194.

Miller, C. T., \& Kaiser, C. R. (2001). A Theoretical Perspective on Coping with Stigma. Journal of Social Issues, 57(1), 73-92. doi:10.1111/0022-4537.00202

Miller, C. T., \& Major, B. (2000). Coping with stigma and prejudice. In T. F. Heatherton, R. E. Kleck, M. R. Hebl, \& J. G. Hull (Eds.), The social psychology of stigma (pp. 243-272). New York: Guilford.

Moore, B. C., Biegel, D. E., \& McMahon, T. J. (2011). Maladaptive coping as a mediator of family stress. Journal of Social Work Practice in the Addictions, 11, 17-39. doi:10.1080/1533256X.2011.544600

Moos, R. H., \& Holahan, C. J. (2003). Dispositional and Contextual Perspectives on Coping: Toward an Integrative Framework. Journal of Clinical Psychology, 59(12), 1387-1403. doi:10.1002/jclp.10229

Nakamura,Y. M., \& Orth, U. (2005). Acceptance as a coping reaction: adaptive or not? Swiss Journal of Psychology, 64, 281-292. doi:10.1024/1421-0185.64.4.281

Neuberg, S. L., Smith, D. M., Hoffman, J. C., \& Russell, F. J. (1994). When we observe stigmatised and "normal" individuals interacting: Stigma by association. Personality and Social Psychology Bulletin, 20(2), 196-209. doi:10.1177/0146167294202007

Norton, T. R., Manne, S. L., Rubin, S., Carlson, J., Hernandez, E., Edelson, M. I., Rosenblum, N., Warshal, D., \& Bergman, C. (2004). Prevalence and predictors of psychological distress among women with ovarian cancer. Journal of Clinical Oncology, 22, 919-926. doi:10.1200/JCO.2004.07.028

Nydegger, R., Nydegger, L., \& Basile, F. (2011). Post-Traumatic Stress Disorder And Coping Among Career Professional Firefighters. American Journal of Health Sciences, 2(1), 11-19.

Onwuegbuzie, A. J., \& Teddlie, C. (2003). A framework for analyzing data in mixed methods research. In A. Tashakkori \& C. Teddlie (Eds.), Handbook of mixed methods in social and behavioral research (pp. 351383). Thousand Oaks, CA: Sage.

Östman, M., \& Hansson, L. (2000a). Family burden and care participation. A test-retest reliability study of an interview instrument concerning families with a severely mentally ill family member. Nordic Journal of Psychiatry, 54, 327-332.

Östman, M., \& Hansson, L. (2000b). Family burden, participation in care and mental health - an 11-year comparison of the situation of relatives to compulsorily and voluntarily admitted patients. International Journal of Social Psychiatry, 46, $191-200$.

Östman, M., \& Kjellin, L. (2002). Stigma by association, psychological factors in family members of people with mental illness. British Journal of Psychiatry, 181, 494-498. doi:10.1192/bjp.181.6.494

Pachankis, J. E. (2007). The psychological implications of concealing a stigma: A cognitive-affective-behavioral model. Psychological Bulletin, 133, 328-345. doi: 10.1037/0033-2909.133.2.328

Pescolido, B. A., Martin, J. K., Long, J. S., Medina, T. R., Phelan, J. C., \& Link, B.C. (2010). A Disease Like Any Other? A Decade of Change in Public Reactions to Schizophrenia, Depression, and Alcohol Dependence. American Journal of Psychiatry, 167, 1321-1330. doi:10.1176/appi.ajp.2010.09121743

Phelan, J. C. (2002). Genetic bases of mental illness-a cure for stigma? Trends Neurosci, 25(8), 430-431. doi:10.1016/S0166-2236(02)02209-9

Phelan, J. C. (2005). Geneticization of deviant behaviour and consequences for stigma: The case of mental illness. Journal of Health and Social Behavior, 46, 307-322. doi:10.1177/002214650504600401

Phelan, J. C., Bromet, E. J., \& Link, B. G. (1998). Psychiatric illness and family stigma. Schizophrenia Bulletin, 24, 115-126. doi:10.1093/oxfordjournals.schbul.a033304

Phelan, J. C., Cruz-Rojas, R., \& Reiff, M. (2002). Genes and stigma: The connection between perceived genetic etiology and attitudes and beliefs about mental illness. Psychiatric Rehabilitation Skills, 6, 159-185. doi:10.1080/10973430208408431

Phelan, J. C., Link, B. G., \&, Dovidio, J. F. (2008). Stigma and prejudice: one animal or two? Social Science \& Medicine, 67(3), 358-367. doi:10.1016/j.socscimed.2008.03.022

Platt, S. (1985). Measuring the burden of psychiatric illness on the family: An evaluation of some rating scales. Psychology Medicine, 15, 383-393. doi: 10.1017/\$0033291700023680

Polit, D. F., \& Beck, C. T. (2010). Essentials of nursing research. Philadelphia, PA: Wolters Kluwer Lippincott Williams \& Wilkins 
Preacher, K. J., \& Hayes, A. F. (2004). SPSS and SAS Procedures for Estimating Indirect Effects in Simple Mediation Models. Behavior Research Methods, Instruments, and Computers, 36(4), 717-731. doi:10.3758/BF03206553

Pryor, J. B., Bos, A. E. R., Reeder, G. D., Stutterheim, S. E., Willems, R. A., \& McClelland, S. (2012). Reactions to stigma-by-association: Relationships to reduced psychological well-being, closeness to stigmatised relatives, and public stigma. Manuscript submitted for publication.

Pryor, J.B., McClelland, S., Reeder, G.D., Bos, A., Stutterheim, S. (2010, May). Affective, Cognitive, and Behavioral Reactions to Having a Stigmatised Family Member. Oral presentation, Midwestern Psychological Association 2010 Meeting, Chicago, US.

Pryor, J. B., Reeder, G. D., \& Monroe, A. E. (2012). The infection of bad company: Stigma by association. Journal of Personality and Social Psychology, 102(2), 224-241. doi:10.1037/a0026270

Pulla, V., Shatté, A., \& Warren, S. (2013). Perspectives on Coping and Resilience. Delhi: Authors Press.

Quinn, D. M. \& Chaudoir, S. R. (2009). Living with a concealable stigmatized identity; The impact of anticipated stigma, centrality, salience, and cultural stigma on psychological distress and health. Journal of Personality and Social Psychology, 97(4), 634-651. doi:10.1037/a0015815

Ramchandani, P., \& Stein, A. (2003). The impact of parental psychiatric disorder on children. British Medical Journal, 327(7409), 242-243. doi:10.1136/bmj.327.7409.242

Read, J., \& Harré, N. (2001). The role of biological and genetic causal beliefs in the stigmatisation of 'mental patients'. Journal of Mental Health, 10(2), 223-235. doi:10.1080/09638230123129

Reed, G. M., Kemeny, M. E., Taylor, S. E., Wang, H. J., \& Visscher, B. R. (1994). Realistic acceptance as a predictor of decreased survival time in gay men with aids. Health Psychology, 13, 299-307. doi:10.1037//0278-6133.13.4.299

Reeder, G. D., \& Pryor, J. B. (2008). Dual Psychological Processes Underlying Public Stigma and the Implications for Reducing Stigma. Poverty and human development, 6(1), 175-186. doi:10.4103/09731229.36546.

Rippetoe, P. A., \& Rogers, R. W. (1987).Effects of components of protection motivation theory on adaptive and maladaptive coping with health threat, Journal of Personality and Social Psychology, 52, 596-604. doi:10.1037//0022-3514.52.3.596

Robinson, E., Rodgers, B., \& Butterworth, P. (2008). Family relationships and mental illness: Impacts and service responses (AFRC Issues No. 4). Melbourne, Australia: Australian Family Relationships Clearinghouse.

Romijn, G., De Graaf, I., \& De Jonge, M. (2010). Kwetsbare kinderen: Literatuurstudie over verhoogde risicogroepen onder kinderen van ouders met psychische of verslavingsproblemen (Research Report Trimbosinstituut AF1060). Retrieved November 28, 2014, from http://www.trimbos.nl/ /media/files/gratis\%20downloads/af1060\%20kwetsbare\%20kinderen_web.ashx

Rosenthal, T. L., Downs, J. M., Arheart, K. L., Deal, N., Downs, A. F. D., \& Rosenthal, R. H. (1991). Similarities and differences on five inventories among mood and anxiety disorder patients. Behaviour Research and Therapy, 29, 239-247. doi:10.1016/0005-7967(91)90113-H

Rüsch, N., Corrigan, P. W., Powell, K., Rajah, A., Olschewski, M., Wilkniss, S., \& Batia, K. (2009). A stress-coping model of mental illness stigma: II. Emotional stress responses, coping behavior and outcome. Schizophrenia Research, 110, 65-71. doi:10.1016/j.schres.2009.01.005

Sales, E. (2003). Family burden and quality of life. Quality of Life Research, 12(1), 33-41.

Saunders, J. C. (2003), Families living with severe mental illness: a literature review, Issues in Mental Health Nursing, 24, 175-198. doi: 10.1080/01612840390160711

Schene, A. H. (1990). Objective and subjective dimensions of family burden, towards an integrative framework for research. Social Psychiatry and Psychiatric Epidemiology, 25, 289-297. doi:10.1007/BF00782883

Schene, A. H., Tessler, R. C. \& Gamache, G. M. (1996). Caregiving in severe mental illness: conceptualization and measurement. In H. C. Knudsen \& G. Thornicroft (Eds.), Mental Health Service Evaluation (pp. 296316). Cambridge: Cambridge University Press.

Seale, C. \& Silverman, D. (1997). Ensuring rigor in qualitative research. European Journal of Public Health, 7(4), 379-384. doi: 
Seeman, M. V. (2013). Spotlight on sibling involvement in schizophrenia treatment. Psychiatry, 76(4), 311-22. doi:10.1521/psyc.2013.76.4.311.

Shah, A. J., Wadoo, O., \& Latoo, J. (2010). Psychological distress in carers of people with mental disorders. British Journal of Medical Practitioners, 3(3), 327-334.

Sibling Support Project. (n.d.). Retrieved December 12, 2014, from http://www.siblingsupport.org/

Skevington, S. M., Lotfy, M., \& O'Connell, K. A. (2004). The World Health Organization's WHOQOL-BREF quality of life assessment: Psychometric properties and results of the international field trial - A report from the WHOQOL group. Quality of Life Research, 13(2), 299-310. doi:10.1023/B:QURE.0000018486.91360.00

Slot, M. A. G., \& Van Aken, N. W. (2010). Psychologie van de adolescentie. Amersfoort, the Netherlands, ThiemeMeulenhoff b.v.

Stålberg, G., Ekerwald, H., \& Hultman, C. M. (2004). Siblings of patients with schizophrenia: Sibling bond, coping patterns and fear of possible schizophrenia heredity. Schizophrenia Bulletin, 30(2), 445-458. doi:10.1093/oxfordjournals.schbul.a007091

Stewart, A., Sherbourne, D., \& Hays, R. (1992). Summary and discussion of MOS measures. In A. L. Stewart \& J. E. Ware (Eds.), Measuring Function and Well-Being: The Medical Outcome Studies Approach (pp. 345371). Durham, NC: Duke University Press.

Strang, V. R., Haughey, M., Gerdner, L. A., \& Teel, C. S. (1999). Respite- A Coping Strategy for Family Caregivers. Western Journal of Nurses Research, 21(4), 450-471. doi:10.1177/019394599902100403

Strauss, A., \& Corbin, J. (1996). Basics of Qualitative Research. Thousand Oaks, CA: SAGE Publications, Inc.

Streisand, B. J., DeWaay, L., Lemorande, R. (Producers), \& Streisand, B. J (Director). (1983). Yent/ [Motion picture]. United States: United Artists.

Struening, E. L., Perlick, D. A., Link, B. G., Hellman, F., Herman, D., \& Sirey, J. A. (2001). Stigma as a Barrier to Recovery: The Extent to Which Caregivers Believe Most People Devalue Consumers and Their Families. Psychiatric Services, 52, 1633-1638. doi:10.1176/appi.ps.52.12.1633

Stutterheim, S. E. (2011). Understanding hiv-related stigma: Social and psychological processes (Doctoral dissertation). Retrieved August 20, 2012, from Maastricht University e-Library. http://arno.unimaas.nl/show.cgi?fid=21066

Stutterheim, S. E., Bos, A. E. R., Pryor, J. B., Brands, R., Liebregts, M., \& Schaalma, H. P. (2011). Psychological and social correlates of HIV status disclosure: The significance of stigma visibility. AIDS Education and Prevention, 23(4), 382-392. doi:10.1521/aeap.2011.23.4.382

Stutterheim, S. E., Bos, A. E. R., Shiripinda, I., De Bruin, M., Pryor, J. B., \& Schaalma, H. P. (2011). HIV-related stigma in African and Afro-Caribbean communities in the Netherlands: Experiences, consequences and coping. Psychology \& Health, 27(4), 395-411. doi:10.1080/08870446.2011.585426

Stutterheim, S. E., Pryor, J. B., Bos, A. E. R., Hoogendijk, R., Muris, P., \& Schaalma, H.P. (2009). HIV-related stigma and psychological distress: The harmful effects of specific stigma manifestations in various social settings. AIDS, 23, 2353-2357. doi:10.1097/QAD.0b013e3283320dce

Sugiura, Y. (2006). Problem focused coping and worry: The mediating role of meta-cognitions. In A. M. Colombus, (Ed.), Leading Edge Research in Cognitive Psychology (pp. 63-77). NY: Nova Science Publishers, Inc.

Swets, M., Middeldorp, C. M., \& Schoevers, R. A. (2009). Heredity and Environmental Influences in Psychiatric Disorders. Tijdschrift voor Psychiatrie, 9, 651-665.

Teddlie, C., \& Tashakkori, A. (2009). Foundations of mixed methods research: Integrating quantitative and qualitative approaches in the social and behavioral sciences. Thousand Oaks CA: SAGE Publications, Inc.

Thomas, D. R. (2006). A general inductive approach for qualitative data analysis. American Journal of Evaluation, 27(2), 237-246. doi:10.1177/1098214005283748

Tsang, H. W. H., Tam, P. K. C., Chan, F., \& Chang, W. M. (2003). Sources of burdens on families of individuals with mental illness. International Journal of Rehabilitation Research, 26(2), 123-130. doi:10.1097/01.mrr.0000070761.13531.a1 
Tuncay, T., Musabak, I., Gok, D. E., \& Kutlu, M. (2008). The relationship between anxiety, coping strategies and characteristics of patients with diabetes. Health and Quality of Life Outcomes, (79), 1-9. doi:10.1186/1477-7525-6-79

University of Michigan Health System. (2014). Siblings of Kids with Special Needs. Retrieved December 8, 2014, from: http://www.med.umich.edu/yourchild/topics/specneed.htm

Van der Sanden, R. L. M. (2014, July). Stigma by Association, Burden and Coping. Oral presentation, $17^{\text {th }}$ European Association of Social Psychology General Meeting, Amsterdam, The Netherlands.

Van Erp, N., Place, C., \& Michon, H. (2009). Familie in de langdurige GGz deel 2: Betrokkenheid in (F)ACT (Research Report No. AF0915). Retrieved from http://www.trimbos.nl/ /media/Themas/4_Behandeling_reintegratie/Familie\%20in\%20de\%20langdurige\%20GGZ\%20deel\%201\%20Interventies.ashx

Vanhauwaert, F. (2010). Het kind en de ouder achter KOPP: Een narratief onderzoek. (Doctoral dissertation). Retrieved December 8, 2014, from http://lib.ugent.be/en/catalog/rug01:001460282

Van Santvoort, F., Hosman, C. M. H., Van Doesum, K. T. M., \& Janssens, J. M. A. M. (2014). Effectiveness of preventive support groups for children of mentally ill or addicted parents: a randomized controlled trial. European Child \& Adolescent Psychiatry, 23, 473-484. doi:10.1007/s00787-013-0476-9

Veit, C. T., \& Ware, J. E. (1983). The structure of psychological distress and well-being in general populations. Journal of Consulting and Clinical Psychology, 51, 730-742. doi:10.1037/0022-006X.51.5.730

Verhaeghe, M., \& Bracke, P. (2012), Associative Stigma among Mental Health Professionals: Implications for Professional and Service User Well-Being, Journal of Health and Social Behavior, 53, 17-32. doi: 10.1177/0022146512439453

Verhaeghe, M., Bracke, P., \& Bruynooghe, K. (2008). Stigmatization and selfesteem of persons in recovery from mental illness: The role of peer support. International Journal of Social Psychiatry, 54, 206-218.

Vogel, D. L., Wade, N. G., \& Hackler, A. H. (2007). Perceived public stigma and the willingness to seek counseling: The mediating roles of self-stigma and attitudes toward counseling. Journal of Counseling Psychology, 54, 40-50. doi:10.1037/0022-0167.54.1.40

Vuksic-Mihaljevic, Z., Mandic, N., Barkic, J., Laufer, D. \& Filakovic, P. (1998). Schizophrenic disorder and family attitudes. European Journal of Psychiatry, 12, 139-152.

Wahl, A., Hanestad, B. R., Wiklund, I., \& Moum, T. (1999). Coping and quality of life in patients with psoriasis. Quality of Life Research, 8, 427-433. doi: 10.1023/A:1008944108101

Wahl, O. F. (1995). Media madness: Public images of mental illness. New Jersey: Rutgers University Press.

Wahl, O. F. (1999). Mental health consumers' experiences of stigma. Schizophrenia bulletin, 25(3), 467-478. doi:10.1093/oxfordjournals.schbul.a033394

Wahl, O. F., \& Harman, C. R. (1989). Family views of stigma. Schizophrenia Bulletin, 15, $131-139$. doi:10.1093/schbul/15.1.131

Weiner, B. (1995). Judgments of responsibility: A foundation for a theory of social conduct. New York: Guilford Press

Weiner, B., Perry, R. P., \& Magnusson, J. (1988). An attributional analysis to stigmas. Journal of Personality and Social Psychology, 55, 738-748.

Werner, P., Goldstein, D., \& Buchbinder, E. (2010). Subjective experience of family stigma as reported by children of Alzheimer's disease patients. Qualitative Health Research, 20, 159-169. doi:10.1177/-1049732309358330

Werner, P., Mittelman, M. S., Goldstein, D., \& Heinik, J. (2012). Family Stigma and Caregiver Burden in Alzheimer's Disease. The Gerontologist, 52(1), 89-97. doi:10.1093/geront/gnr117

West, M. L., Yanos, P. T., Smith, S. M., Roe, D., \& Lysaker, P. H. (2011). Prevalence of Internalized Stigma among Persons with Severe Mental Illness. Stigma Research and Action. Stigma Res Action.1(1): 3-10. doi:10.5463/sra.v1i1.9

Wight, R. G., Aneshensel, C. S., Murphy, D. A., Miller-Martinez, D., \& Beals, K. P. (2006). Perceived HIV stigma in AIDS caregiving dyads. Social Science \& Medicine, 62(2), 444-456. doi:10.1016/j.socscimed.2005. 06.004

Wirth, J. H., \& Bodenhausen, G. V. (2009). The role of gender in mental illness stigma: A national experiment. Psychological Science, 20, 169-173. doi:10.1111/j.1467-9280.2009.02282.x 
Wood, W., \& Eagly, A. H. (2012). Biosocial construction of sex differences and similarities in behavior. In M. P. Zanna \& J. M. Olson (Eds.), Advances in Experimental Social Psychology (pp. 55-123 ). San Diego, CA: Academic Press. doi:10.1016/B978-0-12-394281-4.00002-7

World Health Organization. (2001). Mental Health: New Understanding, New Hope (The World Health Report 2001). Retrieved from World Health Organization website: http://www.who.int/whr/2001/en/whr01_en.pdf?ua=1

World Health Organization. (2014). Mental Disorders (Fact sheet $N^{\circ} 396$, October 2014). Retrieved November 23, 2014, from World Health Organization website: http://www.who.int/mediacentre/factsheets/fs396/en/

Xia, P., Li, N., Hau, K., Liu, C., \& Lu, Y. (2012). Quality of life of Chinese urban community residents: a psychometric study of the mainland Chinese version of the WHOQOL-BREF. BMC Medical Research Methodology, 2012, 12-37. doi:10.1186/1471-2288-12-37

Zauszniewski, J. A,. Bekhet, A. K., \& Suresky M. .J. (2008). Factors associated with perceived burden, resourcefulness, and quality of life in female family members of adults with serious mental illness, Journal of the American Psychiatric Nurses Association, 14, 125-13. doi:10.1177/1078390308315612

Zeidner, M., \& Saklofske, D. (1996). Adaptive and maladaptive coping. In M. Zeidner, \& N. Endler, (Eds.), Handbook of coping: Theory, research, applications (pp. 505-531). Oxford, England: John Wiley \& Sons.

Zhao, X., Lynch, J. G., \& Chen, Q. (2010). Reconsidering Baron and Kenny: Myths and Truths about Mediation analysis. The Journal of Consumer Research, 37(2), 197-206. doi:10.1086/651257 

SUMMARY 



\section{SUMMARY}

People with mental illness not only suffer under the direct consequences of their disorder, they often suffer under a number of additional problems in their family life, their living environment, and their workplace as a result of their condition as well. People with mental illness, for example, regularly experience stigmatising reactions from the people around them because of their mental illness. Previous research has even shown that people with mental illnesses are one of the most stigmatised groups in our society. Stigmatising reactions, however, may not only be geared to the person with the stigmatised condition, but often also to people who are associated with him or her. This is called stigma by association.

In this dissertation, stigma by association among family members of people with mental illnesses is explored. First, the underlying processes and the manifestations of stigma by association are studied. The experiences and the consequences of stigma by association among family members of people with mental illness are examined as well. The influence of specific characteristics of family members of people with mental illness (i.e., familial relationship, gender, co-residence, and the belief that mental illness is hereditary) on their experiences of stigma by association is also examined in this dissertation. Finally, the effect of fourteen different coping strategies used by family members to prevent, reduce, or adapt to experiences and consequences of stigma by association is studied.

In the introduction of this dissertation (chapter 1 ), an overview of the existing literature is given and findings related to the processes of public stigma, stigma by association, family burden and coping are discussed. The implications and dilemmas that stigma by association may entail for family members of people with mental illness are also discussed in the context of the existing literature. To conclude, this chapter presents this dissertation's main research questions.

Chapter 2 presents the results of a quantitative study among 527 family members of people with mental illness. The purpose of this study was to gain insight into the relationships between public stigma, stigma by association, psychological well-being, and the perceived closeness to one's family member with mental illness. The extent to which the type of familial relationship (parent, child, cousin, aunt, partner, etc.) between the person with mental illness and his or her family member influence these relationships was also explored. The influence of the extent to which participants believe mental illnesses to be hereditary on these relationships was investigated as well. The results show that family members of people with mental illnesses regularly face stigma by association. Stigma by association appears to be correlated with reduced psychological well-being and reduced perceived closeness towards family members 
with mental illness. Experiences of stigma by association among immediate family members of people with mental illness (parent, child, sibling, spouse) show a strong association with decreased psychological well-being as well, more than is the case with extended family members (uncle, aunt, nephew, niece). Family members who believe mental illness is hereditary also appear to have more stigma experiences and more psychological distress.

Chapter 3 discusses a qualitative study in which 23 family members of people with mental illness were interviewed about their experiences of stigma by association and the consequences thereof. In this study, family members indicated that indeed they experienced stigma by association. They reported being treated negatively, not being taken seriously, being blamed, and being held responsible for their family members' mental illnesses and deviant behaviour. Family members also reported avoidance, rejection, abandonment, and negative social consequences, such as altered relationships with family members, alienation from friends, the loss of friends, disrupted social relationships, and a smaller social network. This study also examined whether familial relationship, gender, and co-residence played a role in family members' experiences of stigma by association. Parents and spouses of people with mental illness appeared to face blame and negative reactions more than others. Wives and mothers reported being accused of being overprotective, thereby causing or maintaining the mental illness of their spouse or child. Siblings and children of people with mental illness mainly reported stigma by association manifest as being seen as deviant by others, being avoided or being socially excluded because of their familial relationship with someone with mental illness. Living together with the family member with mental illness appeared to play a role in the experiences of stigma by association by family members as well; the familial relationship thus seemed to be so evident that keeping it secret or selectively disclosing this familial relationship was difficult. Nonetheless, many siblings reported taking efforts to selectively disclose or conceal their familial relationship with a person with mental illness, especially in puberty and adolescence. Lastly, more than half of the participants in this study reported experiencing stigma by association in their interactions with mental health professionals and civil servants.

Chapter 4 discusses qualitative research in which 23 immediate family members of people with mental illnesses were interviewed. Their experiences of stigma by association (negative treatment, not being taken seriously, social exclusion, altered familial relationships and friendships) and family burden (quarrels, time-consuming caregiver activities, financial problems, and reduced career opportunities) were extensively described in these interviews. This study also explored the various coping strategies family members of people with mental illnesses used to prevent, reduce, or to adapt to the negative consequences of stigma by association and family burden. The findings showed that family members tend to apply both problem-focused as emotion-focused 
coping strategies. The problem-focused coping strategies reported tended to be aimed at finding and organising social, instrumental, or emotional support, while the emotionfocused coping strategies reported were often directed at avoiding, escaping, or suppressing stigmatised situations.

Chapter 5 describes the results of a quantitative study among 503 immediate family members of people with mental illness. This study examined the connections between stigma by association, family burden, psychological distress and quality of life of family members of people with mental illness. In this study, family members reported their experiences of stigma by association and family burden, and the consequences these experiences have for them and their families. The various ways in which family members cope with the experiences and consequences of stigma by association and family burden were also examined. Fourteen coping strategies were studied to determine their mediating effect on the relationships between stigma by association, family burden, psychological distress, and quality of life. The results demonstrated both complementary and competitive mediating effects of coping on the relationships between stigma by association, family burden, psychological distress, and quality of life. Several problem-focused coping strategies appeared to prevent or reduce the negative impact of stigma by association and family burden, while most of the emotion-focused coping strategies examined were found to be associated with an increase in the negative impact of stigma by association and family burden.

Finally, in chapter 6 , the main findings of the reported studies are integrated and the scientific contribution of these studies to the field of stigma by association is discussed. First, this chapter focuses on the underlying processes, experiences, and consequences of stigma by association among family members of people with mental illnesses. Second, the impact that specific relevant family member characteristics have on the relationships between their experiences of stigma by association and the negative consequences of stigma by association is described. Thirdly, the coping strategies family members use to prevent, reduce, or adapt to their experiences and the consequences of stigma by association are discussed. Finally, the strengths and limitations of the studies under discussion are examined, the practical implications of these studies are outlined, and suggestions for future research are made. 

SAMENVATTING 



\section{SAMENVATTING}

Mensen met een psychische aandoening ondervinden naast de directe gevolgen van deze aandoening vaak ook nog een aantal bijkomende problemen in hun gezinsleven, hun woon- en leefomgeving en op hun werkplek als gevolg van deze aandoening. Zo worden zij vanwege hun psychische aandoening bijvoorbeeld regelmatig geconfronteerd met stigmatiserende emoties en reacties vanuit de mensen in hun omgeving. Uit eerder onderzoek is zelfs gebleken dat mensen met een psychische aandoening tot één van de meest gestigmatiseerde groepen in onze samenleving behoren. Stigmatiserende reacties en emoties richten zich echter niet alleen op de persoon met de stigmatiserende conditie maar vaak ook op mensen die met hem of haar geassocieerd worden, wat stigma by association wordt genoemd.

In dit proefschrift wordt het fenomeen 'stigma by association van familieleden van mensen met een psychische aandoening' nader onderzocht. Allereerst worden de onderliggende processen en de verschijningsvormen van stigma by association bestudeerd. Ook worden de ervaringen met en consequenties van stigma by association voor familieleden van mensen met een psychische aandoening onderzocht. De invloed van specifieke kenmerken van familieleden (i.e., familierelatie, sekse, het al dan niet samenwonen met het familielid met een psychische aandoening, en het al dan niet overtuigd zijn van de erfelijkheid van psychische aandoeningen) op hun ervaringen met stigma by association worden eveneens in dit proefschrift bestudeerd. Tot slot wordt het effect van een veertiental verschillende copingstrategieën die familieleden hanteren om hun ervaringen met en de gevolgen van stigma by association te voorkomen, te verminderen of zich daaraan aan te passen, onderzocht.

In de introductie van dit proefschrift wordt een overzicht van de literatuur en de bestaande bevindingen met betrekking tot de processen van stigma, stigma by association, family burden, en coping behandeld. Ook wordt stil gestaan bij de gevolgen en dilemma's die stigma by association volgens de literatuur en de bestaande inzichten voor familieleden van mensen met een psychische aandoening met zich mee kunnen brengen. Afsluitend geeft dit hoofdstuk de belangrijkste onderzoeksvragen van dit proefschrift weer.

In hoofdstuk 2 worden de resultaten van een kwantitatief onderzoek onder 527 familieleden van mensen met een psychische aandoening behandeld. Het doel van dit onderzoek was om inzicht te verkrijgen in de verbanden tussen stigma, stigma by association, psychologisch welbevinden én de persoonlijke betrokkenheid van mensen bij hun familielid met een psychische aandoening. Hierbij werd ook onderzocht in hoeverre het type familierelatie tussen de persoon met een psychische aandoening en zijn familielid, invloed heeft op genoemde verbanden. Ook werd onderzocht in hoeverre de mate 
waarin familieleden overtuigd zijn van de erfelijkheid van psychische aandoeningen invloed hebben op hun ervaringen met en de gevolgen van stigma by association. Uit deze studie blijkt dat familieleden van mensen met een psychische aandoening zeer regelmatig met stigma by association geconfronteerd worden. Stigma by association blijkt in dit onderzoek samen te hangen met een verminderd psychologisch welbevinden en met een verminderde betrokkenheid van familieleden bij hun familielid met een psychische aandoening. Ook blijkt dat stigma by association bij directe familieleden (ouder, kind, broer, zus, partner) sterker samenhangt met een verminderd psychologisch welbevinden dan bij indirecte familieleden (oom, tante, neef, nicht). Familieleden die er van overtuigd zijn dat psychische aandoeningen erfelijk zijn, blijken eveneens meer stigmatiserende ervaringen en minder psychologisch welbevinden te hebben.

Hoofdstuk 3 bespreekt een kwalitatief onderzoek waarin 23 familieleden van mensen met een psychische aandoening geïnterviewd zijn over hun ervaringen met stigma by association en de gevolgen daarvan. In dit onderzoek gaven familieleden eveneens aan dat zij met stigmatiserende emoties en reacties vanuit de mensen in hun omgeving geconfronteerd werden. Zij rapporteerden dat zij regelmatig negatief bejegend werden, zich niet serieus genomen voelden en hen verweten werd dat zij verantwoordelijk waren voor de psychische aandoening en het afwijkend gedrag van hun familielid. Familieleden rapporteerden te worden ontweken, te worden afgewezen, en in de steek gelaten te worden maar ook andere negatieve sociale consequenties, zoals veranderde relaties met familieleden, vervreemding en het verlies van vrienden, ontwrichte sociale relaties en een steeds kleiner wordend sociaal netwerk. Onderzocht werd in hoeverre de familierelatie, sekse én het al dan niet samenwonen met het betreffende familielid een rol speelden bij de ervaringen met stigma by association door familieleden van mensen met een psychische aandoening. Ouders en partners blijken met name met verwijten en negatieve reacties geconfronteerd te worden. Echtgenotes en moeders blijken daarnaast vaak het specifieke verwijt te krijgen dat zij té beschermend zijn en daardoor de psychische aandoening van hun partner of kind veroorzaken dan wel dat die aandoening daardoor blijft voortbestaan. Broers, zussen en kinderen van mensen met een psychische aandoening blijken vooral te maken te hebben met vormen van stigma by association waarbij zij als 'vreemd' worden gezien en waarbij zij door de mensen in hun directe omgeving worden ontweken of buitengesloten. Het al dan niet samenwonen met een familielid met een psychische aandoening lijkt eveneens een rol te spelen bij de ervaringen met stigma by association door familieleden. De familierelatie wordt door het samenwonen blijkbaar duidelijker waarneembaar, waardoor het geheim houden of selectief onthullen van deze familierelatie wordt bemoeilijkt. Het selectief onthullen of geheimhouden van de relatie die iemand met een met een familielid met een psychische aandoening heeft, lijkt vooral een belangrijke rol te spelen bij broers en zussen van mensen met een psychische aandoening en dan met name tijdens hun puberteit en adolescentie. Tot slot, gaf meer dan de helft van de deelnemers aan 
dit onderzoek aan nog altijd geconfronteerd te worden met stigma by association door professionals in de geestelijke gezondheidszorg en ambtelijke functionarissen.

Hoofdstuk 4 behandelt eveneens kwalitatief onderzoek waarin 23 directe familieleden van mensen met een psychische aandoening geïnterviewd zijn. Hun ervaringen met stigma by association (b.v. negatief benaderd worden, niet serieus genomen worden, sociaal uitgesloten worden, negatieve veranderingen in familierelaties en vriendschappen) en family burden (b.v. ruzies, tijdrovende en stressvolle situaties en zorgtaken, ontwrichting van het gezin, financiële problemen en verminderde carrièrekansen), werden in deze interviews uitgebreid door hen beschreven. In deze studie zijn ook de verschillende copingstrategieën bestudeerd die familieleden van mensen met een psychische aandoening toepassen om de negatieve gevolgen van stigma by association en family burden te voorkomen, te verminderen of zich daaraan aan te passen. Familieleden blijken zowel probleemgerichte- als emotiegerichte copingstrategieën toe te passen. Probleemgerichte copingstrategieën lijken daarbij vooral gericht op het zoeken en organiseren van sociale-, instrumentele- of emotionele steun, terwijl (de meeste) emotiegerichte copingstrategieën er veelal op gericht lijken te zijn om de stigmatiserende situatie zo veel mogelijk te vermijden, te ontvluchten of te verdringen.

In hoofdstuk 5 worden de resultaten van een kwantitatief onderzoek naar de verbanden tussen stigma by association, family burden, psychologisch welbevinden en de kwaliteit van leven onder 503 directe familieleden van mensen met een psychische aandoening beschreven. In dit onderzoek rapporteerden familieleden over de verschillende ervaringen met en gevolgen van stigma by association en family burden voor henzelf en hun gezinsleden. De verschillende manieren waarop familieleden met de negatieve gevolgen van stigma by association en family burden omgaan werden hierbij eveneens in beeld gebracht. Veertien afzonderlijke copingstrategieën zijn bestudeerd op hun mediërend effect op de verbanden tussen stigma by association, family burden, psychologisch welbevinden en kwaliteit van leven. In deze studie werden zowel 'complementaire' als 'competitieve' mediërende effecten van coping op de verbanden tussen stigma by association, family burden, psychologisch welbevinden en kwaliteit van leven aangetroffen. Verschillende probleemgerichte copingstrategieën blijken de negatieve impact van stigma by association en family burden te voorkomen of te verminderen terwijl de meeste emotiegerichte copingstrategieën worden geassocieerd met een toename van de negatieve gevolgen van stigma by association en family burden.

Hoofdstuk 6 integreert de belangrijkste bevindingen uit de gerapporteerde studies. De wetenschappelijke bijdrage van deze studies op het gebied van stigma by association worden hier eveneens besproken. Ten eerste wordt dieper ingegaan op de onderliggende processen, manifestaties, ervaringen en consequenties van stigma by association van familieleden van mensen met een psychische aandoening. Ten tweede wordt aan- 
dacht besteed aan de invloed die specifieke kenmerken van familieleden op de relaties tussen hun ervaringen met en de negatieve consequenties van stigma by association hebben. Ten derde wordt stil gestaan bij de copingstrategieën die familieleden gebruiken om hun ervaringen met en de gevolgen van stigma by association te voorkomen, te bestrijden of zich daaraan aan te passen. Tot slot worden de sterke kanten en beperkingen van de gerapporteerde studies besproken, worden de praktische implicaties van deze studies behandeld en worden suggesties voor vervolgonderzoek gedaan. 


\section{VALORISATION}





\section{VALORISATION}

In this valorisation addendum, a discussion of the additional scientific and practical value of the studies presented in this dissertation is provided.

\section{Public stigma}

Public stigma is a set of negative expectations and reactions of members of the general public towards an individual person or group of persons, and it is, nowadays, seen as an attribute that results in widespread social disapproval. Hence, public stigma contains cognitive, affective, and behavioural elements which reflect the perceiver's perspective.

\section{Stigma by association}

Public stigma may "spill over" from being directed at persons with a stigmatised condition to people associated with them (e.g., family, friends, or companions). This phenomenon is called courtesy stigma, family stigma, or stigma by association.

Public stigma and stigma by association are severe problems for people with mental illness and their family members. Stigma by association diminishes family members' psychological well-being and quality of life, and it hampers the recovery of people with mental illness. Moreover, both public stigma and stigma by association are seen and experienced as a gross social injustice. This dissertation explored and described underlying processes, experiences, and consequences of stigma by association among family members of people with mental illness. It also described the effect of four characteristics (familial relationship, gender, co-residence, and whether one is convinced of the heredity of mental illnesses) of family members of people with mental illness on their experiences and consequences of stigma by association. Lastly, this dissertation presented results on coping strategies family members of people with mental illness use to prevent, reduce, or adapt to the stigmatised condition or situation.

Although the presented studies in this dissertation are cross-sectional, a better understanding of stigma by association, and its underlying processes, experiences, and consequences can likely contribute to more adequate support, information, education, interventions, and stigma reduction programs for family members of people with mental illness. 


\section{RELEVANCE}

The World Health Organization estimates that over 450 million people worldwide suffer from mental illness; some estimates even mention numbers of 500 million and more. As such, a large number of people suffer from mental illness. Subsequently, the number of family members of people with mental illness must be enormous as well as the number of family members that may (potentially) experience stigma by association.

\section{Scientific relevance}

The studies presented in this dissertation increase knowledge about stigma by association among family members of people with mental illness, its underlying processes, experiences, and consequences. The outcomes of these studies can stimulate further research and program development on the experiences and the detrimental effects of stigma by association. The results of the studies presented in this dissertation may also be transferred to adjacent research domains (e.g. industrial and organisational psychology, developmental psychology).

\section{Social relevance}

Stigma by association, as public stigma, is seen and experienced as a social injustice. It also negatively affects the well-being and quality of life of family members of people with mental illness. It may cause family members of people with mental illness to withdraw from social networks and from their family member with mental illness. Furthermore, they may diminish their social support provision to this family member during periods of hospitalisation and thereafter. Both withdrawal and diminished support have detrimental effects for people with mental illness as their family support system is usually one of the last that remains standing. In this way, withdrawal and diminished family support negatively affects the chances of recovery for the family member with mental illness and, as such, enlarges mental illness related burden and costs to society. The direct and indirect effects of stigma by association may, therefore, not only be harmful for both the person with mental illness and his or her family members, but also for society at large, socially and economically.

\section{Economic relevance}

Stigma by association negatively affects the psychological, physical, emotional, and functional health of family members of people with mental illness, and it may subsequently hamper the recovery and therefore lengthen the time in treatment needed by people with mental illness. Consequently, health care costs for the person with mental illness, his or her family members, and society at large may increase due to stigma by 
association. Additionally, family members of people with mental illness may also suffer psychological and physical health problems due to stigma by association, and they may experience productivity losses in the workplace due to these health problems or experience the inability to concentrate on the job, due to the stigmatisation of their family member or oneself. The results reported in this dissertation on coping with stigmatisation may also be transferred to the workplace (e.g., coping with workplace mobbing). Future research can aim to determine how costs can be reduced by stigma reduction programs.

\section{TARGET GROUPS}

The research results in this dissertation can be of interest to the academic community, family members of people with mental illness, people with mental illness, mental health professionals, support and interest groups, policy makers, civil servants, psychology students, and employers.

\section{Academic community}

The phenomenon of stigma by association among family members of people with mental illness has received comparatively little attention in empirical research. This dissertation advances insight regarding the underlying processes, experiences, and consequences of stigma by association. This dissertation also adds to the limited literature on coping with stigma by association among family members of people with mental illness (i.e., aged 18 years or older). Further research may inform us explicitly on the effect of stigma by association among young family members of people with mental illness (aged younger than 18 years) and on the coping strategies they use to prevent, reduce, or adapt to the negative impact of stigma by association.

\section{Family members of people with mental illness}

For family members of people with mental illness, the results can indicate that knowledge of the underlying processes, manifestation, experiences, and consequences of stigma by association is important and that coping with stigma by association is especially important. The extended knowledge of stigma by association presented in this dissertation can be integrated in the development of education programs, interventions on stigma by association, and stigma reduction programs. The findings on coping strategies in this dissertation can help family members develop new and more effective coping skills and encourage them to dispose of maladaptive coping skills. Furthermore, the findings show how individual characteristics of family members can affect their experiences and consequences of stigma by association. These findings emphasise the 
importance of an individual approach and support provision to each individual family member of people with mental illness.

\section{People with mental illness}

Knowledge about the experiences and consequences of stigma by association is relevant for people with mental illness as well as their family members. People with mental illness can become more aware of their family members' experiences of stigma by association, its consequences, and the ways in which these experiences and consequences of stigma by association may negatively affect the lives, attitudes, and family roles of family members within their family.

\section{Mental health professionals and civil servants}

Mental health professionals should be educated and informed about family members' experiences of stigma by association and the consequences thereof. They should explicitly be informed about the detrimental consequences stigma by association may have for family support towards the family member with mental illness and, consequently, for the recovery of this family member. Moreover, some experiences of stigma by association take place in the field of mental health services and thus pertain to mental health professionals and civil servants; the underlying cognitions, emotions, and behaviour of stigma by association should also be combatted within mental health care.

\section{Support and interest groups}

Members of support and interest groups were included in this research. The results of this dissertation may therefore be of specific interest to them. They may bring these findings and the subjects of stigma and stigma by association to the attention of policymakers, and put them on the policy agenda.

\section{Policy makers}

Stigma and stigma by association should become part of policy makers' and politicians' agendas primarily because stigma by association is a social injustice and also because of the high costs the experiences and consequences of stigma by association impose on society (e.g., increased health costs, productivity loss, etc.).

\section{Employers}

Employers may be confronted with the consequences of stigma by association. Employees with a family member with mental illness may have experiences of stigma by 
association, encounter psychological distress, and experience a diminished well-being that may result in concentration problems or absenteeism. Therefore, employers should be informed about stigma by association and its consequences. Employees may perform better when there is a possibility to talk about their home situation, their family member with mental illness, their sorrows, and their experiences of stigma by association, especially if experiences of stigma by association occur at the workplace.

\section{Psychology students}

Psychology students may encounter cognitions, emotions, and attitudes related to experiences of stigma by association among family members of people with mental illness in their future profession. They may also experience stigma by association themselves because of their work as a mental health professional. They should, therefore, be informed and educated on the subject of stigma by association, its consequences, and on coping strategies that may be effective in preventing or targeting stigma by association. Furthermore, it is important that they are aware of their own stigmatising cognitions, emotions, and attitudes.

\section{ACTIVITIES/PRODUCTS}

The findings in this dissertation can be used for adjusting or extending existing educational programs and training sessions, or for the development of new ones for people with mental illness, their family members, mental health professionals, civil servants, and other experts in the field of mental health care. The effects of individual characteristics (familial relationship, gender, co-residence, and the belief that mental illness is hereditary) on family members' experiences and consequences of stigma by association add specific knowledge, and should be taken into account in these programs and training sessions. The knowledge about adaptive and maladaptive coping strategies can be transferred to the development of practical and effective coping skills.

The results of this dissertation can be used to further develop various products and services. Five user groups and related activities/products can be roughly distinguished:

1. Academic community
a. The results of the studies in this dissertation can be presented at conferences within the academic community.
b. Knowledge transfer to science programs on stigmatisation and (mental) health.
c. The development of additional courses and workshops on the subjects of stig- matisation and stigma by association.

2. Family members of people with mental illness, people with mental illness, and support and interest groups 
a. The results of the studies in this dissertation can be presented at conferences among support and interest groups.

b. The development of additional training programs on stigmatisation, stigma by association, and coping strategies.

c. Consulting (knowledge transfer) on the subjects of stigma and stigma by association.

3. Mental health professionals, psychology students, and civil servants

a. The results of the studies in this dissertation can be presented at conferences on psychiatry, psychology, and mental health care.

b. Additional courses and training programs can be developed on the subjects of stigmatisation, stigma by association, and the effects of individual characteristics of family members of people with mental illness on the experiences and consequences of stigma.

c. Workshops on the stigmatisation of professionals in mental health care can be organised, as well as on mental health care professionals' own emotions, prejudice, stereotypes, and stigmatising behaviour towards people with mental illness and their family members

d. Consulting (knowledge transfer) on these subjects.

4. Policy makers

a. The results of the studies in this dissertation can be presented at conferences and debates on (mental) health care, employability, and the labour market

b. Consulting on the subjects of stigmatisation, stigma by association, and mental health care.

5. Employers and employers' associations

a. The results of the studies in this dissertation can be presented at conferences on employability, working environments, and occupational health services.

b. Consulting and coaching on the subjects of stigmatisation at the workplace, of specific professions, and on stigmatised occupational diseases.

\section{INNOVATION}

The findings in this dissertation give more insight into experiences and consequences of stigma by association among family members of people with mental illness, and contribute to the still limited literature on stigma by association. Furthermore, detailed and further insight is given into the effects of individual characteristics of family members of people with mental illness, and coping strategies family members use in relation to experiences and consequences of stigma by association. The results of this dissertation offer substantial input for developing, adjusting, or extending research programs, training sessions, and workshops about mental illnesses, stigmatisation, and stigma by association. We will seek to further implement the results and knowledge of the studies 
presented in this dissertation not only in the field of mental health care, but also in adjacent research domains and subjects (e.g. workplace mobbing, absenteeism reduction programs, return-to-work or work reintegration programs). Consultancy and knowledge-transfer on the subjects of stigmatisation, stigma by association, health care, and return-to-work can be offered, as can participation in public debate.

\section{SCHEDULE AND IMPLEMENTATION}

Several support groups, supported employment organisations, sheltered workshops, social enterprises, and mental health institutions have expressed interest in information and education on the subject of stigma by association. Conference talks will be given in the coming year. Further research on stigma and stigma by association among vocational populations in Dutch emergency and rescue services and the Dutch armed forces is currently the subject of talks with these services and the armed forces. Research methods and procedures will be made suitable for research on stigma and stigma by association among these vocational populations and implementation will continue in the coming years. 



\section{ACKNOWLEDGEMENTS}



Completing the requirements for this dissertation has certainly not been all my own doing. Therefore, I would like to take the opportunity to thank a number of people that have greatly contributed to the realisation of this dissertation.

First, I would like to thank my PhD supervisors, Gerjo Kok and John Pryor, for the confidence they demonstrated in me by wanting to act as my supervisors. The same holds true for my two co-supervisors, Arjan Bos and Sarah Stutterheim. I would like to give special thanks to Arjan and Sarah for their intensive guidance and support, with respect to both research and the English language. Gerjo, John, Arjan and Sarah, I have always found our conversations and meetings to be stimulating, challenging and insightful, and the more informal gatherings were fun and 'tasty.' Gerjo, John, Arjan and Sarah's tireless enthusiasm and commitment, as well as their continuous focus on the "human aspect," are contagious. They not only stimulate in-depth scientific research, but also an intrinsic understanding of the circumstances in which people with mental health problems, and their family members, may find themselves.

A special 'thank you' is in order to all of the participants in the different studies for their cooperation, openness, and courage, including the respondents, the interviewees, the caregivers, mental health organisations, and support groups for people with mental illness and their families. I hope that this dissertation will contribute to the realisation of a "full and inclusive citizenship" for people with mental illness, and to the development of adequate support and guidance for their family members.

I also would like to thank Koen, Ellen and Niels; not only did they have to miss their father often, but they also contributed to the realisation of this dissertation with their questions, reflections, and critical comments.

Finally, I would like to thank my wife Janny, and express my admiration for her. In 2003 when I had just defended my master thesis at Erasmus University in Rotterdam and we walked down the stairs together, we heard my former thesis supervisors, Ton and Lucas, talk about 'a PhD', mainly to tease her. We both thought, "No way, we have had enough!" Still, 12 years later, we are on the eve of the defence of this dissertation. Throughout each study, and for this dissertation as well, you knew that it would be an investment of several years, that my part in the family would be limited, and that my study load would partly be yours. Since Carnival ' 85 , not a day went by that I did not realise how lucky I have been with you and the kids. Therefore, I dedicate this dissertation to you, because you have encouraged me to be a better person every day over the past several decades. 

CURRICULUM VITAE 

Remko van der Sanden was born on August 18, 1961 in Oosterhout, the Netherlands. He joined the Dutch Police Force in 1981 and attended higher professional education at the Institute for Advanced Police Studies in Warnsveld. He is currently CEO at the ATEAgroup, a conglomerate of social enterprises, sheltered workshops, and organisations for disabled and unemployed people in the Netherlands. Bachelor's degrees were obtained at the Institute for Organisation Studies in Tilburg (1993) and the Institute for Economics in 's-Hertogenbosch (1996). Van der Sanden received his Master of Science in Business Administration at the Erasmus University in Rotterdam (2003) and his Master of Science in Psychology at the Open University in Heerlen (2006). During this time, he led several semi-public organisations and reorganisations. In 2010, he initiated the research reported in this dissertation at Maastricht University, Department of Work and Social Psychology. He completed this dissertation research in 2015. He continues his involvement and research on mental illness stigma (e.g., PTSD) and stigma by association among vocational populations, such as law enforcers, armed forces, and emergency and rescue services. 


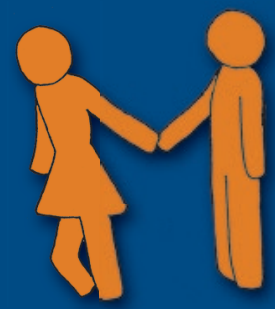

